Aus dem Institut für Neuroanatomie

(Prof. Dr. med. J. Staiger)

im Zentrum Anatomie

der Medizinischen Fakultät der Universität Göttingen

\title{
Schichtenspezifische Charakterisierung von \\ Parvalbumin-exprimierenden Neuronen im primären somatosensorischen Kortex der Maus
}

\author{
INAUGURAL-DISSERTATION \\ zur Erlangung des Doktorgrades \\ für Zahnmedizin \\ der Medizinischen Fakultät der \\ Georg-August-Universität zu Göttingen
}

vorgelegt von

Bettina Anna Pater

aus Köln

Göttingen 2019 
Dekan:

Referent/in:

Ko-Referent/in:

Drittreferent/in:
Prof. Dr. med. W. Brück

Prof. Dr. Jochen Staiger

Prof. Dr. Tim Gollisch

Prof. Dr. Rainer Mausberg

Datum der mündlichen Prüfung: 20.07.2020 
Hiermit erkläre ich, die Dissertation mit dem Titel "Schichtspezifische Charakterisierung von Parvalbuminexprimierenden Neuronen im primären somatosensorischen Kortex der Maus" eigenständig angefertigt und keine anderen als die von mir angegebenen Quellen und Hilfsmittel verwendet zu haben.

Göttingen, den

(Unterschrift) 


\section{Inhaltsverzeichnis}

ABBILDUNGSVERZEICHNIS .......................................................................II

TABELLENVERZEICHNIS ......................................................................... IV

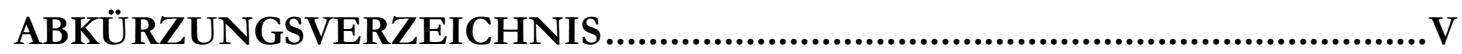

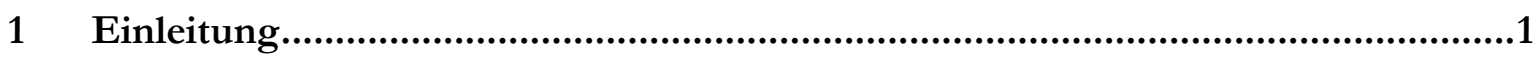

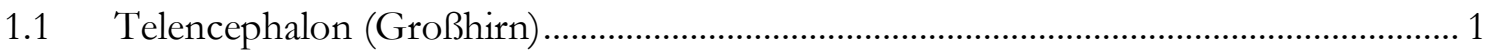

$1.2 \quad$ Barrel-Kortex (Somatosensorischer Kortex) …............................................................ 3

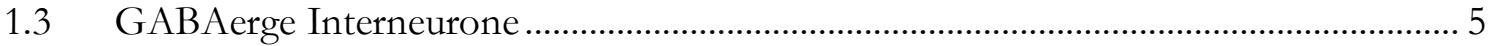

1.3.1 $\quad P V$-exprimierende Interneurone ………............................................................

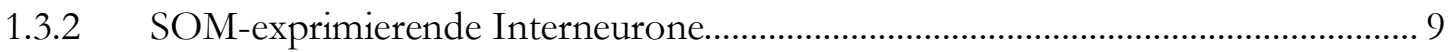

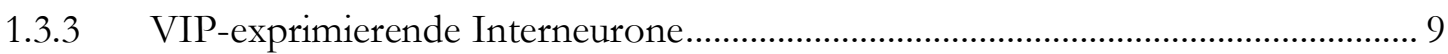

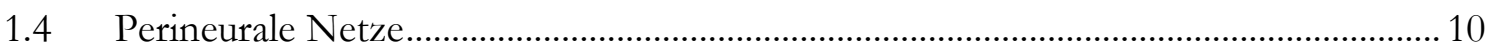

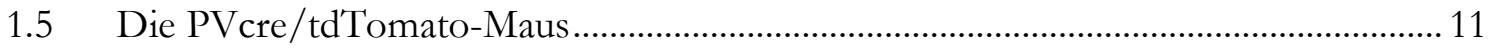

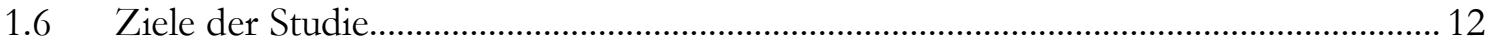

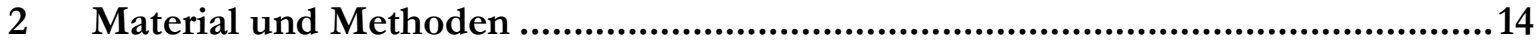

2.1 Versuchstiere und Vorbereitung der Hirnschnitte............................................................ 14

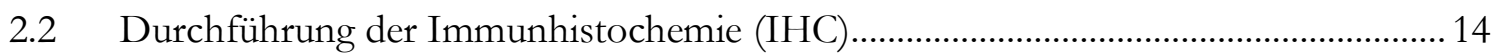

2.3 Durchführung der Fluoreszenz-in-situ-Hybridisierung (FISH) .................................. 15

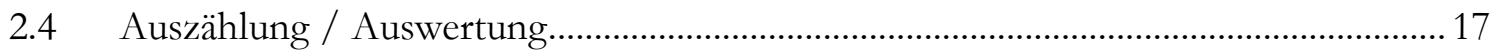

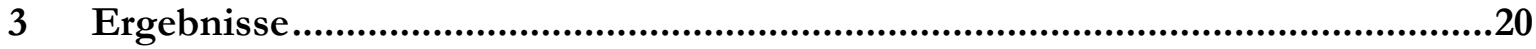

3.1 Sind die tdTomato-positiven Zellen in der PVcre-Maus auch Parvalbumin-positiv $(+)$ ?

3.2 Sind die tdTomato-positiven Zellen im Barrel Kortex exzitatorisch oder inhibitorisch? .26

3.3 Kolokalisation von tdTomato-positiven Zellen mit inhibitorischen Markern.............. 34

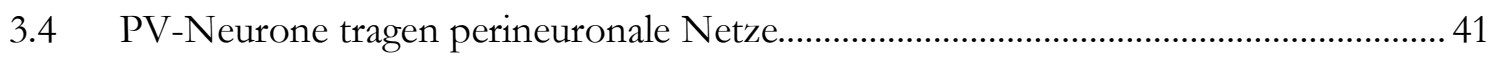




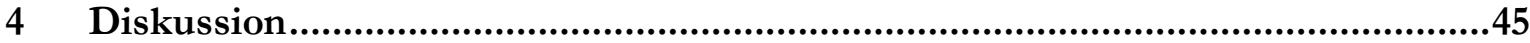

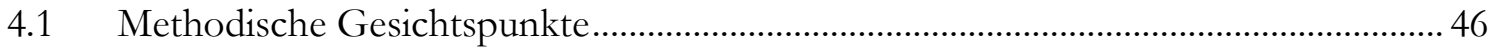

4.1.1 Immunhistochemie und Fluoreszenz-in-situ-Hybridisierung ............................... 46

4.1.2 Vergleich von Epifluoreszenz mit ApoTome und Konfokalmikroskopie........... 47

4.1.3 Auszählung und manuelles Einzeichnen der Schichten.......................................... 50

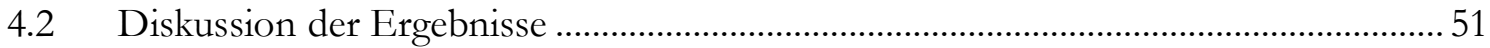

4.2.1 PV-exprimierende Interneurone der PVcre-Maus.................................................... 51

4.2.2 Verteilung der tdTomato-Zellen in der PVcre-Maus ............................................... 56

4.2.3 PVcre/tdTomato-Zellen und perineuronale Netze ................................................ 58

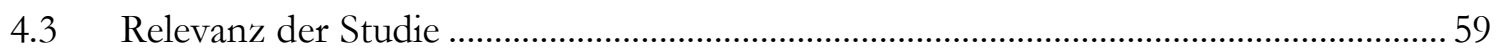

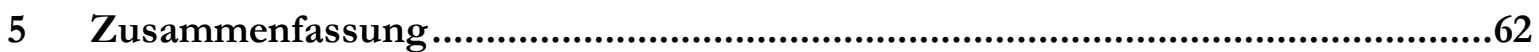

6 Anhang

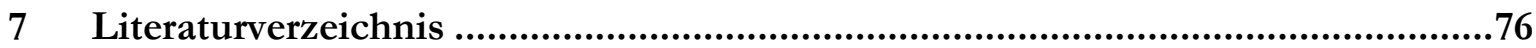




\section{ABBILDUNGSVERZEICHNIS}

Abbildung 1.1: Sechsschichtiger Aufbau des Barrel-Kortex......................................................... 2

Abbildung 1.2: Hirnschnitt einer Maus in einem Frontalschnitt.................................................... 5

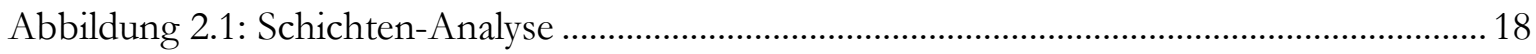

Abbildung 3.1: Ausschnitte einer PV-Antikörper-Färbung zur Darstellung PV-positiver Zellen im Bereich des Barrel-Kortex der PVcre/tdTomato-Maus

Abbildung 3.2: Kolokalisation zwischen den PV/tdTomato-Zellen und dem PV-Antikörper...23 Abbildung 3.3: Schichtspezifische Verteilung der tdTomato-Zellen im Barrel-Kortex der

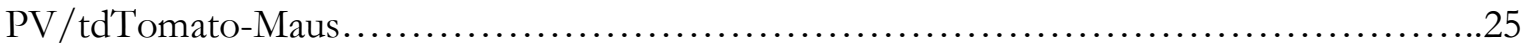

Abbildung 3.4: FISH zur Darstellung Gad1-positiver Zellen im Bereich des Barrel-Kortex der PVcre/tdTomato-Maus

Abbildung 3.5: Kolokalisation zwischen den tdTomato-Zellen und der Gad1-Sonde.

Abbildung 3.6: FISH zur Darstellung Vglut1-positiver Zellen im Bereich des Barrel-Kortex der PVcre/tdTomato-Maus 31

Abbildung 3.7: Kolokalisation zwischen tdTomato und der V glut1-Sonde 32

Abbildung 3.8: Kolokalisation zwischen tdTomato mit der Gad1- und Vglut1-Sonde. 33

Abbildung 3.9: FISH zur Darstellung VIP-positiver Zellen im Bereich des Barrel-Kortex der PVcre/tdTomato-Maus .35

Abbildung 3.10: Kolokalisationen zwischen tdTomato und der Vip-Sonde. 37

Abbildung 3.11: FISH zur Darstellung SOM-positiver Zellen im Bereich des Barrel-Kortex der PVcre/tdTomato-Maus .38

Abbildung 3.12: Kolokalisationen zwischen tdTomato und der Sst-Sonde. 39

Abbildung 3.13: Vergleich der prozentualen schichtenspezifischen Kolokalisation zwischen den tdTomato-Zellen und dem PV-Antikörper, der Sst-sowie der Vip-Sonde...... 40

Abbildung 3.14: Ausschnitte einer WFA-Färbung zur Darstellung perineuronaler Netze im Bereich des Barrel-Kortex der PVcre/tdTomato-Maus .42

Abbildung 3.15: Kolokalisationen zwischen tdTomato und WFA. 493

Abbildung 3.16: Kolokalisation zwischen tdTomato und dem Antikörper WFA in \% 494 Abbildung 4.1: Konfokale Aufnahme der IHC und FISH aus Schicht IV........ 49 


\section{TABELLENVERZEICHNIS}

Tabelle 6.1: Anzahl der tdTomato-Zellen und der aPV-positiven Zellen in den einzelnen Schichten des Barrel-Kortex

Tabelle 6.2: Prozentuale schichtspezifische Verteilung der Zellen, die nur tdTomato-positiv sind oder nur den Antikörper PV tragen sowie die kolokalisierten Zellen 64

Tabelle 6.3: Schichtspezifische Verteilung der kolokalisierten tdTomato-Zellen mit aPV 65

Tabelle 6.4: Prozentuale schichtspezifische Verteilung und die gesamte Anzahl an tdTomatoZellen

Tabelle 6.5: Anzahl der tdTomato-Zellen und der Gad1-positiven Zellen in den einzelnen Schichten des Barrel-Kortex 656

Tabelle 6.6: Anzahl der tdTomato-Zellen und der Vglut1-positiven Zellen in den einzelnen Schichten des Barrel-Kortex 66

Tabelle 6.7: Schichtspezifische Verteilung der kolokalisierten tdTomato-Zellen mit Gad1- und Vglut1-Sonde 67

Tabelle 6.8: Anzahl der tdTomato-Zellen und der VIP- sowie SOM-positiven Zellen in den einzelnen Schichten des Barrel-Kortex 68

Tabelle 6.9: Anzahl an kolokalisierten tdTomato-Zellen mit der Vip- und Sst-Sonde.

Tabelle 6.10: Schichtspezifische Verteilung der kolokalisierten tdTomato-Zellen mit der Vipoder Sst-Sonde.

Tabelle 6.11: Anzahl der Zellen in der WFA-Färbung in den einzelnen Schichten des BarrelKortex 70

Tabelle 6.12: Prozentuale schichtspezifische Verteilung der Zellen, die nur tdTomato-positiv oder nur WFA-positiv sind sowie die kolokalisierten Zellen

Tabelle 6.13: Schichtspezifische Verteilung der kolokalisierten tdTomato-Zellen mit dem WFASignal

Tabelle 6.14: Ergebnisse des Mann-Whitney-Ranksummentests 712

Tabelle 6.15: Verwendete Sonden der FISH .75 


\section{ABKÜRZUNGSVERZEICHNIS}

$3 \mathrm{~V}$

Amy

AS

CSPG

D3V

DAPI

DEPC

DNA

FISH

GABA

GAD/Gad

HB

HC

IHC

LV

PB

PBS

PFA

PNN

PV

RNA

RNAse

Rt

RT

S1BF

$\mathrm{SOM} / S_{s t}$

SSC

TB

TBS

TBST dritter Ventrikel

Amygdala

Antisense

Chondroitinsulfat-Proteoglykan

dorsaler dritter Ventrikel

4,6-Diamidin-2-Phenylindol

Diethylpyrocarbonat

Desoxyribonukleinsäure, desoxyribonucleic acid

Fluoreszenz-in-situ-Hybridisierung

$\mathrm{y}$-Aminobuttersäure, $y$-aminobutyric acid

Glutamatdecarboxylase

Hybridisierungspuffer, bybridisation buffer

Hippocampus

Immunhistochemie

lateraler Ventrikel

Phosphatpuffer, phosphate buffer

phosphatgepufferte physiologische Kochsalzlösung, phosphate-buffered saline

Paraformaldehyd

perineuronale Netze

Parvalbumin

Ribonucleinsäure, ribonucleic acid

Ribonukleasen

Nucleus reticularis thalami

Raumtemperatur

primärer somatosensorischer Barrel-Kortex, primary somatosensory barrel field

Somatostatin

Standard-Saline-Citratpuffer, standard saline citrate buffer

Tris-Puffer, Tris buffer

Tris-gepufferte physiologische Kochsalzlösung, Tris-buffered saline

Tris-gepufferte physiologische Kochsalzlösung mit Triton X-100, Tris buffered

saline with triton $X-100$

VGLUT/Vglut vesikulärer Glutamattransporter 
VIP/Vip vasoaktives intestinales Polypeptid

VPM Nucleus ventralis posteromedialis thalami, ventral posteromedial nucleus

WFA/WFL Wisteria floribunda Agglutinin/Wisteria floribunda Lectin

wm

weiße Substanz, white matter

ZNS

Zentralnervensystem 


\section{Einleitung}

\subsection{Telencephalon (Großhirn)}

Das zum Zentralennervensystem der Säugetiere gehörende Telencephalon (Großhirn) macht den größten Anteil der fünf Hirnabschnitte aus. Aus der Phylogenese heraus kann man es in drei Bereiche einteilen: den Paläokortex, den Archikortex und den jüngsten Teil, den Neokortex. Generell besteht der Kortex aus der sich außen befindenden Großhirnrinde, der grauen Substanz (Substantia grisea) und dem sich Innen befindenden Mark, der weißen Substanz (Substantia alba). Die Großhirnrinde vom Menschen ist ca. 2-5 mm dick. Hier befinden sich verschiedene Zelltypen sowie deren Fortsätze. Aus histologischer Sicht wird der Kortex in den Isokortex und den Allokortex gegliedert. Der kleinere Anteil, der Allokortex besteht aus meistens drei Schichten. Dagegen weist der Isokortex, der circa 80 \% des menschlichen Gehirns ausmacht, eine kontinuierliche sechsschichtige zelluläre Zusammensetzung auf. Dieser Aufbau wird durch die Verteilung der Pyramidenzellen und Nichtpyramidenzellen charakterisiert. Der sechsschichtige neuronale Aufbau des Isokortex wird außen von der Pia mater, einer Bindegewebsschicht, abgeschlossen. Ausgehend von ihr folgen die verschiedenen Schichten (Laminae): die Molekularschicht (I), die äußere Körnerschicht (II), die äußere Pyramidenschicht (III), die innere Körnerschicht (IV), die innere Pyramidenschicht (V) sowie die multiforme Zellschicht (VI) (Guy und Staiger 2017; Brodmann und Gary 2006; Schierloh et al. 2003; Forssmann und Heym 2013). Die einzelnen Schichten können sich in verschiedenen Gehirnregionen in ihrer Ausprägung voneinander unterscheiden. In der Hirnforschung dient die Maus (Mus musculus) als Modellsystem des Säugetiergehirns. Anhand des somatosensorischen Kortex stellen sich die neokortikalen Schichten in der Maus wie folgt dar: 


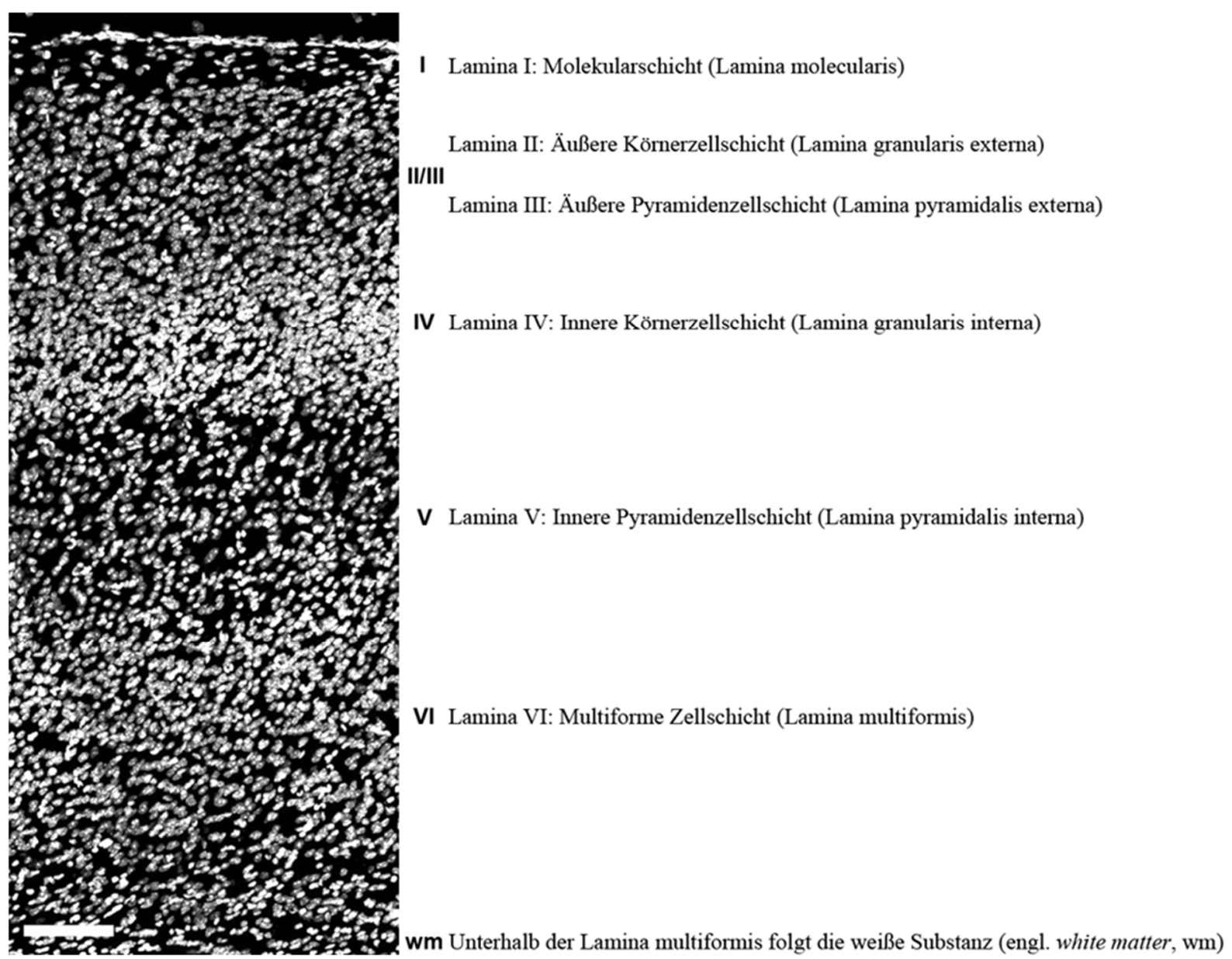

\section{Abbildung 1.1: Sechsschichtiger Aufbau des Barrel-Kortex}

Darstellung der neokortikalen Schichten (Laminae) in einem $40 \mu \mathrm{m}$ dicken Frontalschnitt im Bereich des somatosensorischen Kortex einer Maus mittels einer Epifluoreszenzaufnahme in der Zellkernfärbung (DAPI); zu sehen sind die einzelnen Schichten (I-VI) in römischen Zahlen angegeben; Schicht Va und $\mathrm{Vb}$ sind in Schicht V zusammengefasst; die weiße Substanz (wm) sowie die Namen der Schichten in Deutsch (Latein); Maßstab $100 \mu \mathrm{m}$ (Scheuer 2015)

Zur Differenzierung der einzelnen neokortikalen Schichten können sowohl die Zelldichte als auch die Zellverteilung genutzt werden. Für den somatosensorischen Kortex der Maus wurde der prozentuale Anteil der Schichten an der Gesamtkortexdicke bestimmt: Schicht I 5,7 \%, Schicht II/III 19,4 \%, Schicht IV 17,2 \%, Schicht V 20,5 \% und Schicht VI 37,2 \% (DeFelipe et al. 2002). Des Weiteren können die kortikalen Schichten aufgrund einer unterschiedlichen Zelldichte charakterisiert werden. Die erste Schicht zeichnet sich durch eine geringe Zelldichte aus. Hier befinden sich zu $94 \%$ GABAerge Nichtpyramidenzellen, Neurogliaforme Zellen und ein sehr geringer Anteil an glutamaterger Cajal-Retzius-Zellen (Prieto et al. 1994; Staiger et al. 2015). In den folgenden Schichten II-VI befinden sich diverse morphologisch unterschiedliche Zelltypen, wie z. B. Chandelierzellen, Korbzellen, Martinotti-Zellen, bipolare/doppeltgebüschte, projizierende und neurogliaforme Zellen (Staiger et al. 2015). 
Zwischen Schicht II und III kann man aufgrund einer gleichmäßigen Zellverteilung im Hirn des Nagetiers keine genaue Unterteilung vornehmen. Aus diesem Grund werden sie zur Schicht II/III zusammengefasst. In diesen Schichten befinden sich Körner- und Pyramidenzellen, die eine gleichmäßige Verteilung aufweisen, sowie vermehrt die GABAergen Chandelierzellen. Die größte Zelldichte wird von der Schicht IV repräsentiert. In ihr befinden sich dicht gepackte kleine Körnerzellen. In der Schicht V sind große und mittelgroße Pyramidenzellen angeordnet. Die Schicht VI beinhaltet kleine gleichmäßig verteilte Zellen (Ren et al. 1992); normale und modifizierte Pyramidenzellen sowie Sternzellen. Eine weitere separate Unterteilung erfolgt in Schicht V. Sie wird vor allem in primär sensorischen Arealen in eine Schicht Va und Vb untergliedert; einerseits aufgrund unterschiedlicher Zellverteilung, andrerseits besitzen sie unterschiedliche funktionale Konnektivitätsmuster (Schubert et al. 2006). Kennzeichnend für die Schicht Va, die in der gesamten Schicht V etwa ein Drittel einnimmt, ist ihre geringe Anzahl mittelgroßer pyramidaler Zellen. Die zelldichtere Schicht $\mathrm{Vb}$ hingegen enthält pyramidenförmige Neurone, die in ihrer Größe variabel sind (Schubert et al. 2006).

\subsection{Barrel-Kortex (Somatosensorischer Kortex)}

Ein Teil des Isokortex ist der primär somatosensorische Kortex. Er ist verantwortlich für die zentrale Verarbeitung der haptischen Wahrnehmung. Diese Region verarbeitet Sinnesmodalitäten wie Druck, Vibration, Berührung, Temperatur und zum Teil auch Schmerzempfindung. Er erhält seine Informationen vor allem aus dem Thalamus, der selbst mit seinen Fasern größtenteils in die Schicht IV (Simons 1978; Staiger et al. 1996; Petersen 2007; Feldmeyer 2012) und in anderen kortikalen Arealen projiziert, sowie aus neuromodulatorischen Zentren.

Durch seine Organisation in kortikale Kolumnen in Schicht IV aufgrund der erhöhten zylindrisch geformten Zelldichte und die markante Zellanordnung, die bei Betrachtung eine Assoziation an Tönnchen (Fässer) hervorruft, bekam dieser somatosensorische Bereich den englischen Namen Barrel Cortex (S1BF). Dieser Bereich ist mikroskopisch in der Schicht IV des Kortex sichtbar und nimmt bei Mäusen eine Fläche von 2,52 $\mathrm{mm}^{2}$ ein. In jeder Hemisphäre kann die Maus um die 170 bis 210 Barrels aufweisen, die jeweils einen Durchmesser von ca. 100-400 $\mu \mathrm{m}$ besitzen. Die zellärmeren Bereiche zwischen den Barrels werden als Septen bezeichnet (Woolsey und Van der Loos 1970). Variationen der Dimensionen an Barrels können zwischen verschiedenen Tierspezies auftreten (Riddle und Purves 1995). 
Die Maus nimmt ihre Umgebung wesentlich über ihre Schnurrhaare (Vibrissen) wahr, sodass die eingehenden Reize über die sensorischen Rezeptoren bis zum primären somatosensorischen Kortex verlaufen.

Die Weiterleitung der taktilen Information von den einzelnen Vibrissen erfolgt stets somatotop geordnet über Hirnstamm und Thalamus bis zum Barrel-Cortex. Man spricht in diesem Zusammenhang auch von der Whisker-to-Barrel-Verschaltung (Petersen 2007; Schubert et al. 2007). Die Sinnenrezeptoren an den Vibrissenwurzeln werden vom Nervus trigeminus und seinen Seitenästen (Nervus infraorbitalis) kontaktiert und dieser leitet die Information zum Nukleus trigeminus im Hirnstamm. Von dort gehen die somatotopen Bahnen zu spezifischen Thalamuskernen (Nukleus ventralis posterior medialis (VPM) und Nukleus posterior medialis (Pom)) und letztlich terminieren die Bahnen in allen Schichten des Barrel-Kortex, wobei eine gewisse Dominanz in Schicht IV existiert (Staiger et al. 2002). Dabei kann bei der somatotopischen Projektion jeder Vibrisse eine funktionelle Kolumne innerhalb des somatosensorischen Kortex zugewiesen werden (Staiger et al. 2002). 


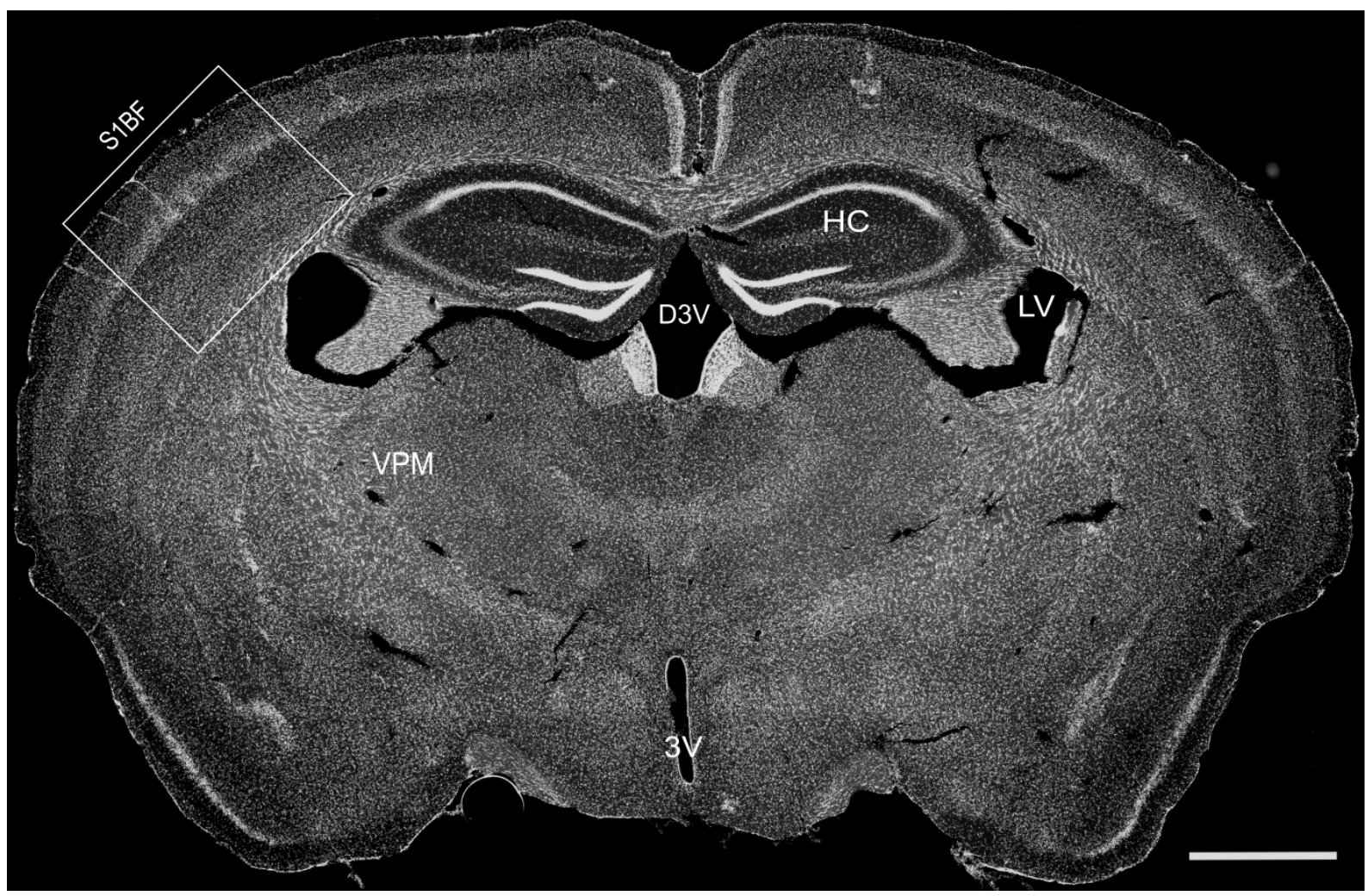

Abbildung 1.2: Frontalschnitt durch das Vorderhirn einer Maus

Darstellung einer Zellkernfärbung (DAPI) eines Frontalschnitts des Mäusehirns in der Epifluoreszenzaufnahme. Gekennzeichnet sind die folgenden Strukturen: durch das Rechteck der Barrel-Kortex $(=\mathrm{S} 1 \mathrm{BF})$, Hippocampus $(\mathrm{HC})$, Nucleus ventralis posteromedialis (VPM), lateraler Ventrikel (LV), dritter Ventrikel (3V) und dorsaler dritter Ventrikel (D3V); Maßstab $1000 \mu \mathrm{m}$.

In diesem neuronalen Netzwerk des somatosensorischen Kortex befinden sich exzitatorische (70-80\%) sowie inhibitorische Zellen. Die Pyramidenzellen der Großhirnrinde stellen die Hauptpopulation der exzitatorischen Neurone dar und verwenden überwiegend den exzitatorischen Neurotransmitter Glutamat. Ihre Besonderheit liegt in ihrer markanten Gestalt, eines dreieckigen Zellkörpers und ihrer Funktion als Projektionsneurone Informationen in andere Gehirnareale weiterzugeben. $\mathrm{Zu}$ den Nichtpyramidenzellen zählt man die exzitatorischen Körnerzellen und die inhibitorischen GABAergen Interneurone.

\subsection{GABAerge Interneurone}

Die inhibitorischen GABAergen Interneurone (20-30\%) machen nur einen geringen Anteil der kortikalen Zellen aus, jedoch sind sie maßgeblich an der Signalprozessierung, an der Modulation kortikaler Funktionen, sowie an der Steuerung und Modifikation eingehender Erregungen beteiligt (Markram et al. 2004). Somit sind sie in der Lage den Informationsfluss in der Hirnrinde zu steuern (Rudy et al. 2011; Tremblay et al. 2016; Feldmeyer et al. 2018). GABAerge 
Interneuronen synthetisieren und lassen an ihren Synapsen den Neurotransmitter GABA frei (Kubota et al. 1994). GABA (y-Aminobuttersäure) ist chemisch gesehen ein Aminosäurederivat und besitzt im Kortex weitgehend eine inhibitorische, d. h. kurzschließende oder hyperpolarisierende Wirkung. Diese Eigenschaft der Interneurone kann eine entscheidende Bedeutung auf die Empfindlichkeit, die Komplexität und die Dynamik des inhibitorischen Systems unabhängig von der Intensität und der Komplexität des Reizes bereitstellen (Markram et al. 2004). Durch die Vielfalt der Synapsen entsteht eine verbesserte Verarbeitungsdynamik innerhalb des Systems und es wird ein Gleichgewicht zwischen dem ständigen Wechsel der inhibitorischen und exzitatorischen Eingänge geschaffen (Markram et al. 2004).

Zur Unterscheidung der inhibitorischen und exzitatorischen Neuronen im Neokortex kann der immunhistochemische Nachweis spezifischer Proteine genutzt werden. Die VglutTransporterfamilie (eng. vesicular glutamate transporter) dient zur Darstellung der Glutamatfreisetzungsfähigkeit und damit von exzitatorischen Zellen (Kehrl et al. 2017), zu denen neben den Pyramidenzellen, die Sternpyramidenzellen sowie die bedornten Sternzellen zählen (Dori et al. 1992). Es werden verschiedene Isoformen des vesikulären Glutamattransporters identifiziert: Vglut 1-3 und der Glutamat/Aspartat-Transporter Sialin. Sie unterscheiden sich in ihrer Zell- und Gewebeverteilung voneinander, sodass sie unterschiedliche physiologische Funktionen erfüllen können (Kehrl et al. 2017). Die Hauptaufgabe der VglutTransporter besteht in der Aufnahme der exzitatorischen Aminosäure L-Glutamat in synaptischen Vesikeln. Angetrieben wird diese Aktivität durch einen elektrochemischen Protonengradienten, der steuernden vaskulären ATPase und durch eine niedrige ChloridionenKonzentration (Thompson et al. 2005; Kehrl et al. 2017). Bei einer Stimulation der Präsynapse durch einlaufende Aktionspotentiale erfolgt die Freisetzung von Glutamat in den synaptischen Spalt.

Hinsichtlich der verschiedenen Isoformen kann Vglut1 als natriumabhängiger Phosphattransporter BNPI (brain-specific Nat-dependent in organic phosphate cotransporter) (Ni et al. 1994) charakterisiert werden. Vglut2 hingegen wurde anfänglich als DNPI (differentiation-associated brain-specific $\mathrm{Na}+$-dependent inorganic phosphate cotransporter) (Aihara et al. 2000) beschrieben. Die Gemeinsamkeit dieser beiden Transporter liegt in ihrer Kinetik (Bellocchio et al. 2000; Takamori et al. 2000). Die zwei Transporter haben zudem Einfluss auf die Speicherung nicht-glutamaterger Transmittern und können die Aufnahme an vesikulärem GABA und Monoamin beeinflussen (Zander et al. 2010). Im adulten Gehirn der 
Maus kann Vglut1 im Hippocampus, Kortex und Cerebellum nachgewiesen werden (Fremeau et al. 2001). Vglut2 kommt dagegen größtenteils im Hirnstamm, in den tiefen cerebellären Kernen und im Thalamus sowie Hypothalamus vor (Fremeau et al. 2001). Die Expression des dritte Subtyps $V$ glut3 ist auf diskrete Zellpopulationen beschränkt (Herzog et al. 2004). Dieser Transporter befindet sich im Gehirn, in der Leber und Niere. In dieser Arbeit wird Vglut1 als Marker zum Nachweis exzitatorischer Zellen im Barrel-Kortex genutzt und dessen mögliche Kolokalisation mit den tdTomato-positiven Neuronen der zu untersuchenden transgenen Maus für PV-exprimierende Neuronen (PVcre-Maus) untersucht.

Die Darstellung der Gruppe inhibitorischer Zellen kann durch die Glutamat-Decarboxylase $(G A D)$ erfolgen. Dies sind Enzyme, die bei einer Decarboxylierung Glutamat zu GABA (GABA = engl. gamma-aminobutyric acid) und $\mathrm{CO}_{2}$ katalysieren. Die zwei Gene GAD1 und GAD2 weisen unterschiedliche Aktivitäten auf und codieren für zwei unterschiedliche, nämlich GAD67 und GAD65, die in bestimmten Zellen im Gehirn exprimiert werden. Die Expression von GAD2 erfolgt zusätzlich in der Bauchspeicheldrüse. Die beiden Formen von $G A D$ unterscheiden sich in ihrer intraneuronalen Verteilung. GAD67 ist im gesamten Neuron verteilt, während GAD65 in erster Linie in den Terminalen der Axone vorkommt (Kaufman et al. 1991). Mittels des Markers Gad1 soll der Nachweis erbracht werden, dass es sich bei den tdTomatopositiven Zellen der PVcre-Maus um inhibitorische Interneurone handelt.

\subsection{1 $P V$-exprimierende Interneurone}

Parvalbumin ist ein Kalzium-bindendes Protein mit einem Molekulargewicht von 9-11 kDa. Durch eine Helix-Loop-Helix Domäne kann es Kalzium binden und gehört wie Calretinin (CR) und Calbindin (CB) zu der Troponin C Proteinfamilie. 40-50 \% der GABAerger Interneuronen exprimieren Parvalbumin im Zytosol (Wonders und Anderson 2006; Xu et al. 2010; Rudy et al. 2011). Die PV-exprimierenden Interneurone sind mitverantwortlich für die Regulierung der neuronalen Plastizität und nehmen Einfluss auf die kortikale Entwicklung während der „kritischen Periode“ (Chevaleyre und Piskorowski 2014). Betrachtet man die Aktionspotentiale der PV-exprimierenden Interneurone, so können sie in die Klasse der schnell feuernden, eng. fast spiking Interneurone eingeteilt werden (Cauli et al. 1997). Zwischen den Parvalbuminexprimierenden Interneuronen erfolgt eine gegenseitige Inhibition (Pfeffer et al. 2013), sie projizieren auf Martinotti-Zellen (MC) (Walker et al. 2016) sowie auf VIP Neuronen (Staiger et al. 1997). Während ihrer physiologisch synchronen Aktivität erzeugen sie Oszillationen, die eine Frequenz von ca. 30-80 Hz besitzen. Diese können als Gammarythmen bezeichnet werden 
(Buzsáki und Draguhn 2004; Cardin et al. 2009; Sohal et al. 2009), von denen angenommen wird, dass sie eine wichtige Rolle bei der Steuerung der Aufmerksamkeit und der Gedächtnisbildung übernehmen (Jensen et al. 2007). PV-exprimierende Interneuronen lassen sich als Zellen charakterisieren, die einen niedrigen Eingangswiderstand besitzen und Aktionspotentiale mit kurzer Halbwertszeit aufweisen (Kawaguchi und Kubota 1997).

Morphologisch können sie in weitere Subgruppen unterteilt werden. Die größte Gruppe der PV-exprimierenden Interneurone stellen die Korbzellen (BCs, Basket Cells) dar und übernehmen als Aufgabe die spezifische Hemmung der Somata und proximalen Dendriten von Pyramidenzellen und anderen PV-Zellen (Tremblay et al. 2016). Sie werden zum Teil nochmals in drei Unterklassen unterteilt: große Korbzellen ( $L B C$ s, large basket cells), kleine Korbzellen (SBCs, small basket cells) und Nestkorbzellen (NBCs, nested basket cells) (Markram et al. 2004). Daneben existiert die Gruppe der Axo-axonische bzw. Chandelierzellen (ChCs, chandelier cells), die als Ziel ausschließlich das Axoninitialsegment von Pyramidenzellen ansteuern (Tremblay et al. 2016). Sie erlangten ihren Namen durch die Bildung von Kronleuchter-ähnlichen synaptischen Kartuschen, mit denen sie auf die Axoninitialsegmente der Pyramidenzellen einwirken (Tremblay et al. 2016). Obwohl die beiden Gruppen der Korb- und Kronleuchterzellen zu dem Typ der schnell feuernden Interneurone gehören, unterscheiden sich ihre elektrophysiologischen Eigenschaften voneinander (Woodruff et al. 2009). Eine letzte noch nicht ausführlich untersuchte Subgruppe, die sich multipolar bursting-Zellen (MBCs) nennt, bei der es sich wahrscheinlich um ein Methodenartefakt handelt, kommen überwiegend in der Schicht II vor und weisen gegenüber den zuvor genannten PV-exprimierenden Interneuronen andere morphologische und elektrophysiologische Eigenschaften auf (Blatow et al. 2003).

Im Neokortex besteht eine Kolokalisation der PV-exprimierenden Interneurone mit GABA und dem Protein Calbindin (D28k) (Kubota et al. 1994). Kubota und Mitarbeiter haben in immunohistochemischen Versuche an Mäusen und Ratten gezeigt, dass PV und SOM keine Kolokalisation aufweisen (Kubota et al. 1994). Hingegen präsentieren andere Studien durch die Verwendung der In-situ-Hybridisierung (ISH), das einige PV-positive Neurone (circa $10 \%$ im somatosensorischen Kortex SOM-mRNA enthalten. Dagegen zeigen PV-Interneurone der Schicht VI mit circa 16,3 \% eine deutliche Überlappung mit den SOM-exprimierenden Interneuronen (Lee et al. 2010). Bei der Betrachtung der PV-exprimierenden Interneuronen und der weiteren Subpopulation der VIP-exprimierenden Interneuronen lagen keine Kolokalisationen im Barrel-Kortex der Maus vor (Scheuer 2015). 


\subsubsection{SOM-exprimierende Interneurone}

Somatostatin besitzt als Peptidhormon endokrine sowie neuronale Funktionen. SOMexprimierende Interneurone wird eine gezielte Inhibition von Dendriten zugeschrieben (Tremblay et al. 2016). Hinsichtlich ihrer elektrophysiologischen Eigenschaften grenzen sie sich $\mathrm{zu}$ den anderen Interneuronen durch ihr kontinuierlich adaptierendes Feuermuster (continous adapting) ab (Halabisky et al. 2006; Ma et al. 2006). Im Gegensatz zu den PV-Interneuronen erfolgt die Inhibition nicht reziprok, sondern auf andere Populationen (Pfeffer et al. 2013). In Bezug auf ihr morphologisches Aussehen können sie in zwei ähnlich zahlreiche Untergruppen, den Martinotti- und der Nicht-Martinotti-Zellen, eingeteilt werden (Tremblay et al. 2016). Martinotti-Zellen besitzen einen stark verzweigten Axonplexus in der Schicht I und kommen vorwiegend in den Schichten II und V vor. Entsprechend ihres inhibitorischen Charakters besteht im Neokortex eine Kolokalisation zwischen GABA und Somatostatin (Kubota et al. 1994). 15-30 \% der Somatostatin-exprimierenden Zellen sind im Neokortex der Maus mit Calretinin kolokalisiert (Tremblay et al. 2016), aber nicht mit den beiden großen Subgruppen der VIP- und PV-exprimierenden Interneuronen (Prönneke et al. 2015). Zusätzlich wurde nachgewiesen, dass einige SOM-Zellen nNOS (neuronal nitric oxide synthase) exprimieren (Tremblay et al. 2016).

\subsubsection{VIP-exprimierende Interneurone}

Die Zellpopulation der VIP-exprimierenden Interneurone kommt überwiegend in den Schichten II/III ( 60\%) des Kortex vor (Prönneke et al. 2015). Eine charakteristische Eigenschaft der VIP-Zellen ist, dass sie andere inhibitorische Interneurone hemmen und über eine disinhibitorische Wirkung Einfluss auf kortikale Schaltkreise nehmen können (Prönneke et al. 2015; Staiger et al. 2004; Feldmeyer et al. 2018).

Unter elektrophysiologischer Betrachtung zeigen die VIP-exprimierenden Zellen häufig ein unregelmäßiges Feuermuster (irregular spiking) (Cauli et al. 1997). Ein anderes wesentliches elektrophysiologisches Merkmal ist ihr relativ hoher Eingangswiderstand, der höher als bei den meisten anderen kortikalen Neurone liegt (Tremblay et al. 2016). Diese Eigenschaft erklärt ihre hohe Empfindlichkeit auf exzitatorische Eingänge. Des Weiteren besitzen die VIPInterneurone die Möglichkeit SOM-Zellen zu inhibieren (Pfeffer et al. 2013; Walker et al. 2016; Karnani et al. 2016). Dies steht in dem Zusammenhang mit der bevorzugten Synapsenbildung von VIP-Neuronen auf SOM-exprimierenden Interneuronen (Tremblay et al. 2016). Charakterisiert man die Interneurone bezüglich ihrer Morphologie, so kann man eine große 
Mehrheit als vertikal orientiert, bipolar dendritisch und den anderen Teil als multipolare Zellen beschreiben (Prönneke et al. 2015; Bayraktar et al. 2000).

Im Hinblick auf die inhibitorischen Eigenschaften wird auch hier eine Kolokalisation zwischen VIP und GABA detektiert (Kubota et al. 1994). Wie bereits beschrieben, kommt es zu keiner Kolokalisation der VIP-exprimierenden Interneurone mit den jeweiligen anderen großen molekularen Subpopulationen, nämlich SOM und PV. Aus diesem Grund können sie als eigenständige, nicht überlappende Subpopulation eingestuft werden (Férézou et al. 2002). Darüber hinaus sind sie entweder mit Calretinin oder mit Cholecystokinin kolokalisiert (Tremblay et al. 2016).

GABAerge Interneurone kann man in drei große Gruppen nach ihren morphologischen, elektrophysiologischen und molekularen Eigenschaften einteilen (Markram et al. 2004). Die molekulare Unterscheidung erfolgt aufgrund ihrer Expression von Neuropeptiden, wie Somatostatin (SOM), Cholecystokinin, vasoaktivem intestinalem Peptid (VIP) und der Gruppe der Kalzium-bindender Proteine, wie Parvalbumin (PV), Calretinin und Calbindin (Markram et al. 2004). GABAerge Interneurone lassen sich letztlich in drei distinkte molekular unterscheidbare Subpopulationen einteilen. Die Parvalbumin-exprimierenden Interneurone stellen mit $40 \%$ die größte Gruppe dar. Alle anderen inhibitorischen Neurone exprimieren entweder Somatostatin oder den ionotropen Serotonin-Rezeptor 3a, mit jeweils 30 \%. Letztere Subgruppe stellt die heterogenste Gruppe dar und kann nochmals in die VIP-exprimierenden (40\%) und nicht-VIP (60\%) Zellen unterteilt werden (Rudy et al. 2011). Dabei weisen die drei Subpopulationen an Interneuronen eine spezifische Verteilung innerhalb der kortikalen Schichten auf (Rudy et al. 2011; Staiger et al. 2015; Feldmeyer et al. 2018).

\subsection{Perineurale Netze}

Perineuronale Netze (PNN) sind gitterartige spezialisierte Strukturen der extrazellulären Matrix, die im Säugergehirn für Stabilität der Synapsen sorgen sollen. Im zentralen Nervensystem können sich PNN um Zellkörper und Dendriten von Neuronen sowie um deren Synapsen befinden. PNN bestehen aus dem Polysaccharid Hyaluronsäure und besitzen darüber hinaus ein Grundgerüst aus Proteogylcanen der Familie der Lecticane. Sie sind bevorzugt negativ (polyanionische Matrix) geladen und enthalten größtenteils Chondroitinsulfat-Proteoglykan (CSPG) (Wang und Fawcett 2012). 
Obwohl die funktionelle Bedeutung der PNNs für lange Zeit ungeklärt war, entdeckte man vor kurzem, dass sie bei der Regulation neuronaler Plastizität, der Erzeugung von $\gamma$-Oszillationen sowie der Neuroprotektion eine wichtige Aufgabe übernehmen (Kwok et al. 2011). Die Entstehung des PNN korreliert mit dem Ende der ,kritischen“ Phase in der Entwicklung des Neokortex (Pizzorusso et al. 2002). Grundlegend gibt es bereits verschiedene experimentelle Ansätze, bei denen eine Entfernung der PNNs zu einer Aktivierung der Plastizität sogar im adulten ZNS führen kann (Wang und Fawcett 2012).

Des Weiteren wird angenommen, dass perineuronale Netze einen Schutz gegen verschiedene neurodegenerative Erkrankungen schaffen (Karetko und Skangiel-Kramska 2009). Andere Studien besagen, dass PNN ebenfalls Schutz der Neurone gegenüber Eisenabsonderung sowie oxidativen Stress bieten (Morawski et al. 2004). Es wurde in diesem Zusammenhang entdeckt, dass die PV-exprimierenden Interneurone ein vermehrtes Vorkommen an Perineuronalen Netzen aufweisen. Dies kommt offensichtlich dadurch zustande, dass die schnell-feuernden PV-Zellen durch ihre erhöhte Aktivität bei der Koordination neuronaler Gruppen einen erhöhten Stoffwechselbedarf und eine hohe mitrochondriale Dichte besitzen, die sie besonders empfindlich auf oxidativen Stress reagieren lässt (Cabungcal et al. 2013).

Zur Visualisierung der Perineuronalen Netze, nutzt man als Marker das Wisteria floribunda Agglutinin (WFA) oder auch Wisteria floribunda Lectin (WFL) genannt, die an die terminalen N-Acetylgalactosaminreste von CSPGs binden können (Young und Williams 1985). In dieser Studie erlaubte fluoreszenzmarkiertes WFA die Darstellung perineuronaler Netze im Bereich des Barrel-Kortex.

\subsection{Die PVcre/tdTomato-Maus}

Die transgene PVcre/tdTomato-Mauslinie wurde in vielen Studien zur Untersuchung jener GABAerger Interneurone, die PV exprimierenden verwendet. Diese Mauslinie wurde durch das Cre/loxP- System, ein Rekombinations-System, generiert. Die Cre/loxP-Technik bietet eine gezielte Modifikation von DNA-Sequenzen, das gezielte Entfernen (Deletion) oder Einfügen (Insertion) von DNA-Sequenzen in lebenden Organismen und damit eine genetische Modifikation durch die Cre-Rekombinase des Bakteriophagen P1. Entdeckt wurde das Phänomen beim Bakteriophagen P1 und wird in der molekularbiologischen Forschung vielfältig genutzt, wie zum Beispiel zur Herstellung gewebespezifischer Knockout-Mäuse. Das Protein Cre-Rekombinase $(C r e=$ Cyclization recombination $)$ kann zwischen spezifischen Basensequenzen, den loxP-Stellen (loxP = locus of $x$ over P1), die als Erkennungssequenz dienen, Spaltungen und 
Neuverknüpfungen der DNA katalysieren. Dazu wird gezielt eine loxP-Stelle vor und hinter der Ziel-DNA-Sequenz eingebaut, sodass im nächsten Schritt die Cre-Rekombinase diese DNASequenz herausschneiden oder in entgegengesetzter Orientierung wieder einbauen kann. Die in dieser Studie verwendeten PVcre/tdTomato-Mäuse wurden durch die Verpaarung von PVcre-

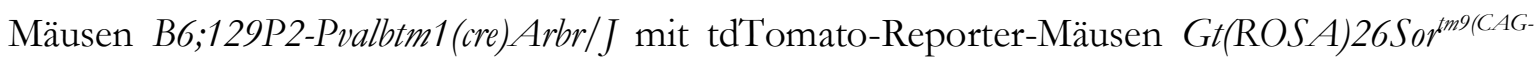
tdTomato $H_{\gtrless}$ erzeugt. In der PVcre/tdTomato-Maus soll die Cre-Rekombinase ausschließlich in Parvalbumin-exprimierenden Zellen aktiv sein. Daher sollte nur in diesen Zellen das zwischen den loxP-Stellen inserierte tdTomato-Protein umgedreht werden und somit transkribiert werden können.

Durch diese spezielle Technik kann die PVcre/tdTomato-Mauslinie erzeugt werden, die eine native tdTomato-Fluoreszenz in den Parvalbumin-exprimierenden Neuronen aufweisen kann. Durch Anregung des tdTomato-Proteins mit Licht grüner Wellenlängen (max. 550nm), wird eine Emission von Licht roter Wellenlängen $(\max .580 \mathrm{~nm})$ hervorgerufen, wodurch die PVexprimierenden Zellen für den Betrachter im Mikroskop sichtbar werden.

\subsection{Ziele der Studie}

GABAerge Interneurone stellen mit 20-30 \% der kortikalen Neurone nur eine kleine, aber für die Informationsverarbeitung wichtige Gruppe dar. Sie lassen sich anhand der Expression des Kalzium-bindenden Proteins Parvalbumin, des Neuropeptids Somatostatin und des ionotropen Serotoninrezeptor (5-HT3a) in 3 distinkte Subgruppen unterteilen. Unterschiede in der Expression dieser und weiterer molekularer Marker wurden genutzt, um zelltypspezifische transgene Mausmodelle zu generieren. Dadurch sind GABAerge Interneurone Gegenstand vieler Studien geworden, die sich deren molekularer oder neurochemischen Charakterisierung, Verschaltung und Funktion annehmen. Es ist jedoch anzumerken, dass bei der Etablierung neuer Mausmodelle für die GABAergen Interneurone weitgehend auf eine gründliche Charakterisierung dieser Mauslinien verzichtet wird.

In dieser Arbeit soll die Mauslinie PVcre hinsichtlich ihrer Expression spezifischer Marker für GABAerge Interneurone und damit auf ihre Spezifität und Sensitivität untersucht werden. Durch die Verwendung der Cre/loxP-Technik wurden PVcre/tdTomato-Mäuse gezüchtet, deren PVcre-Zellen mit dem fluoreszierenden Protein tdTomato markiert sind.

Mittels immunhistochemischer Färbungen sowie der Fluoreszenz-in-situ-Hybridisierung wurden die PVcre-Zellen im somatosensorischen Kortex hinsichtlich der Expression spezifischer Interneuronmarker (Parvalbumin (PV), Somatostatin (SOM) und vasoaktiven 
intestinalen Polypeptid (VIP) untersucht. Des Weiteren soll der Nachweis erbracht werden, dass es sich bei den PVcre-Zellen um inhibitorische Zellen handelt. Dafür wurde die Synthese der Glutamatdecarboxylase 1 (Gad1) für die inhibitorischen Neurone und des vesikulären Glutamattransporters 1 (Vglut1) als exzitatorischen Marker verwendet. Basierend auf vorangegangenen Studien, die ein besonders zahlreiches Vorkommen perineuronaler Netze (PNN) um Parvalbumin-exprimierender Zellen beobachteten, wurde das Lektin Wisteria floribunda Agglutinin (WFA) eingesetzt, um die PVcre-Zellen diesbezüglich zusätzlich zu charakterisieren.

Ziel der Studie ist es, eine Aussage über die schichtspezifische Verteilung der Parvalbuminexprimierenden Zellen im Bereich des Barrel-Kortex sowie deren potenzielle Kolokalisation mit dem Interneuronmarkern SOM bzw. VIP und den exzitatorischen ( $V$ glut 1$)$ und inhibitorischen (Gad1) Markern treffen zu können. Ebenfalls soll der Nachweis über das spezifische Vorkommen PNN an PV-positiven Neuronen erbracht werden. Die Auswertung erfolgt anhand frontaler Hirnschnitte, die mit Hilfe der Epifluoreszenz-basierten Mikroskopie aufgenommen worden sind. Die Hirnschnitte wurden anschließend Software-unterstützt ausgezählt und die erhobenen Daten quantitativ und statistisch in tabellarischer sowie graphischer Form dargestellt. 


\section{Material und Methoden}

\subsection{Versuchstiere und Vorbereitung der Hirnschnitte}

Für die durchgeführten Experimente wurden acht adulte männliche PVcre/tdTomato-Tiere mit einem Gewicht von 20-30 g verwendet. Die Tiere wurden unter Standardbedingungen in der Zentralen Tierexperimentellen Einrichtung der Universitätsmedizin Göttingen im 12h Tag/12 h Nacht Rhythmus gehalten. Alle Experimente erfolgten unter Einhaltung des deutschen Tierschutzgesetzes (Stand 2013).

Die Versuchstiere wurden durch ausgebildetes Fachpersonal der Arbeitsgruppe perfundiert und für den Schneidevorgang vorbereitet. Hierfür wurden die Tiere durch eine intraperitoneale Injektion von Ketamin (0,1 ml pro $10 \mathrm{~g}$ Körpergewicht) narkotisiert, durch Eröffnung des Thorax und einer transkardialen Perfusion mit 4\%-igem Paraformaldehyd (PFA) in 0,1 M Phosphatpuffer (PB, pH 7,4) fixiert. Nach der Perfusion wurden die Gehirne aus dem Schädel präpariert und postfixiert $\left(2 \mathrm{~h}\right.$ in PFA bei $\left.4^{\circ} \mathrm{C}\right)$. Die Gehirne für die Immunhistochemie wurden nach erfolgter Postfixierung zweimal für jeweils fünfzehn Minuten (min) in 0,1 M pH 7,4 PB gewaschen und anschließend bis zum Schneideprozess bei $4{ }^{\circ} \mathrm{C}$ in PB gelagert. Die Gehirne für die Fluoreszenz-in-situ-Hybridisierung wurden nach der Postfixierung mit Kryoprotektionslösung (20\%ige Sucroselösung in 0,1 M PB mit 0,9\% NaCl, PBS, pH 7,4) behandelt und über Nacht bei $4{ }^{\circ} \mathrm{C}$ gelagert. Am folgenden Tag wurden die Gehirne mit Isopentan bei $-40{ }^{\circ} \mathrm{C}$ schockgefroren und bis zum Schneiden bei $-80{ }^{\circ} \mathrm{C}$ gelagert.

\subsection{Durchführung der Immunhistochemie (IHC)}

Von vier PVcre/tdTomato-Tieren wurden unter Verwendung eines Vibratoms (VT 1200S, Firma Leica, Wetzlar, Deutschland) $40 \mu \mathrm{m}$ dicke Frontalschnitte im Bereich des Barrel-Kortex der Mäuse angefertigt, in PB $(0,1 \mathrm{M}, \mathrm{pH} 7,4)$ gesammelt und bei $4{ }^{\circ} \mathrm{C}$ gelagert. Die Schnitte wurden vor der Färbung wie folgt vorbehandelt: 2x15 min in 0,05 M Tris Puffer (TB, pH 7,6), 2x15 min in 0,05 M Tris gepufferte Saline-Lösung (TBS, pH 7,6) sowie 2x15 min in TBS mit 0,5 \% Triton X-100 (TBST, pH 7,6) bei Raumtemperatur (RT). Anschließend erfolgte die Blockierung unspezifischer Antigene bei RT auf dem Schüttler für $90 \mathrm{~min}$ in 0,25\%igem Rinderserumalbumin (Carl Roth, Karlsruhe, Deutschland) und 10\%igem Normalen Ziegenserum (Normal Goat Serum, NGS der Firma Jackson Immuno-Research Laboratories, West Grove, Pennsylvania, USA) in TBST (0,05 M, pH 7,6), verdünnt in TBST. 
Der primäre Antikörper (rb-a-PV 25 (Swant, Martly, Schweiz) 1:5000; die Färbelösung biotinconjugated lectin Wisteria floribunda agglutinin (Sigma-Aldrich, München, Deutschland)) 1:2000 wurden mit der Blockierlösung wie angegeben verdünnt.

Je primärem Antikörper sowie je WFA-Lösung wurden drei Gehirnschnitte auf einem Schüttler für 72 Stunden bei $4{ }^{\circ} \mathrm{C}$ inkubiert. Es folgten Waschschritte von $1 \times 5$ min und $4 \times 15$ min mit TBST (0,05 M, pH 7,6) bei RT. Der sekundäre Antikörper goat-anti-rabbit Alexa 488, 1:500 (Life Technologies, Darmstadt, Deutschland) wurde in TBST (0,05 M, pH 7,6) verdünnt und es erfolgte die Inkubation der Schnitte auf einem Schüttler für 4 Stunden bei RT. Weitere Spülvorgänge bei RT schlossen sich an: $1 \times 5$ min und 2x15 min in TBST $(0,05 \mathrm{M}, \mathrm{pH} 7,6)$ und 1x15 min TBS (0,05 M, pH 7,6). Die Zellkerne der Gehirnschnitte wurden daraufhin mit 4'-5diamidino-2-phenylindole (DAPI, 1:1000, Life Technologies, Darmstadt, Deutschland) auf einem Schüttler bei RT für 5 min in TBS $(0,05 \mathrm{M}, \mathrm{pH} 7,6)$ angefärbt.

Anschließend wurden die Schnitte bei RT 1x15 min in TBS (0,05 M, pH 7,6) sowie 2x15 min in TB $(0,05 \mathrm{M}, \mathrm{pH} 7,6)$ gewaschen. Auf einem Schüttler wurden die Schnitte über Nacht bei $4{ }^{\circ} \mathrm{C}$ in TB $(0,05 \mathrm{M}, \mathrm{pH} 7,6)$ gelagert. Am folgenden Tag konnten die Hirnschnitte auf einen Objektträger gezogen, leicht angetrocknet und mit Aqua Poly-Mount (Polyscience, Warrington, Pennsylvania, USA) und eingedeckt werden.

\subsection{Durchführung der Fluoreszenz-in-situ-Hybridisierung (FISH)}

Die durchgeführten Versuche basieren auf der mRNA-Fluoreszenz-in-situ-Hybridisierung. Wichtig hierfür sind RNAse-freie Materialien, so dass alle verwendeten Materialien vier Stunden auf $180{ }^{\circ} \mathrm{C}$ erhitzt worden sind. Weiterhin wurden ausschließlich RNAse-freie Einwegartikel benutzt. Die verwendeten Lösungen wurden zur Reinigung von RNAsen mit 0,1\% Diethylpyrocarbonat (DEPC) behandelt und am darauffolgenden Tag bei $121{ }^{\circ} \mathrm{C}$ für $20 \mathrm{~min}$ autoklaviert.

Die bei $-80{ }^{\circ} \mathrm{C}$ gelagerten Mausgehirne wurden für die Herstellung $40 \mu \mathrm{m}$ dicker Hirnschnitte im Bereich des Barrel-Kortex durch Tissue-Tek O.C.T. Compound (Sakura, Alphen aan den Rjin, Niederland) bei $-50{ }^{\circ} \mathrm{C}$ in der Halterung des Kyrostaten (Leica CM3050 S, Nußloch, Deutschland) fixiert und anschließend bei $-18{ }^{\circ} \mathrm{C}$ geschnitten. Insgesamt wurden von vier PVcre/tdTomato-Mäusen Hirnschnitte angefertigt, bei RT in PBS-Lösung $(\mathrm{pH} 7,4)$ gesammelt, in Lochplatten sortiert und anschließend $1 \times 15$ min in PBS $(0,1 \mathrm{M}, \mathrm{pH} 7,4)$ gespült. Es folgte die endogene Peroxidase-Blockierung für 20 min in 1\%iges $\mathrm{H}_{2} \mathrm{O}_{2}$ in Methanol sowie ein Quenching für 8 min mit 0,2 M HCL und Andauen der Schnitte für 10 min mit Proteinase K, gelöst in Tris- 
HCL/EDTA (10 mg/ml). Zwischen den einzelnen Schritten wurden die Schnitte jeweils 2x2 min mit PBS-Lösung $(0,1 \mathrm{M}, \mathrm{pH} 7,4)$ gewaschen. Anschließend erfolgte eine 20-minütige Nachfixierung bei $0{ }^{\circ} \mathrm{C}$ in 4\%igem PFA in $0,1 \mathrm{M} \mathrm{PB}(\mathrm{PH} 7,4)$.

Die weitere Vorbehandlung bestand aus 10 min Quenching in Triethanolamine /HCL (0,1 M, $\mathrm{pH}$ 8) bei dem unter Schütteln jeweils zweimal 2,4 $\mu \mathrm{l} / \mathrm{ml}$ Acetsäureanhydrid zugegeben wurde. Daraufhin wurden die Schnitte 3x15 min in PBS-Lösung (0,1 M, pH 7,4) und 1x15 min in 2xSSC (Standard-Saline-Citratpuffer, pH 7,1) gespült. Für die Inkubierung wurden die Schnitte 1x15 min lang in einer 2xSSC-Lösung im Hybrididisierungspuffer 1:1 (HB $=50 \%$ deionisiertes Formamid, 4xSSC, $5 \%$ Dextransulfat, $250 \mu \mathrm{g} / \mathrm{ml}$ HSS-DNA, $100 \mu \mathrm{g} / \mathrm{ml} \mathrm{t}-\mathrm{RNA}$ sowie 1x Denhardts-Reagenz) bei RT und auf dem Rotationsschüttler behandelt. Danach folgte für eine Stunde die Prähybridisierung der Schnitte in Hybridisierungspuffer bei $55^{\circ} \mathrm{C}$. Die eingesetzten Sonden wurden in Hybridisierungspuffer angesetzt und, um sicherzustellen, dass die RNA einzelsträngig vorliegt, wurden sie in einem Hitzeschock auf $95{ }^{\circ} \mathrm{C}$ für 2 min erwärmt. Anschließend wurden die Sonden den vereinzelten Schnitten zugegeben und über Nacht bei 55 ${ }^{\circ} \mathrm{C}$ hybridisiert. In der hier durchgeführten Studie kamen folgende Sonden zur Anwendung: Somatostatin (Sst AS, 29.05.2015), vasoaktives intestinales Polypeptid (Vip AS, 24.05.2015), vesikulärer Glutamattransporter 1 (Vglut1 AS, 24.06.2015) und Glutamatcarboxylase 1 (Gad1(67) AS hergestellt am 24.06.15) (Tab. 6.15).

Am darauffolgenden Tag wurden die Schnitte mehrmals in verschiedenen Lösungen gewaschen: $2 \mathrm{x} 15 \mathrm{~min}$ bei RT in $2 \mathrm{xSSC}, 2 \mathrm{x} 30 \mathrm{~min}$ bei $55^{\circ} \mathrm{C}$ in $2 \mathrm{xSSC} / 50 \%$ Formamid (FA) sowie $2 \times 5$ min bei $55^{\circ} \mathrm{C}$ in $2 x S S C$. Für die komplette Beseitigung der Reste der eingesetzten Sonden wurden die Schnitte 1x35 min bei $37^{\circ} \mathrm{C}$ in RNAse A (4 $\mu \mathrm{g} / \mathrm{ml}$, Marcherey-Nagel, Düren, Deutschland), einem Enzym, das die Katalyse von Phosphodiesterbindungen in RNA-Ketten durch hydrolytische Spaltung ausführt, gelöst und in 2xSSC behandelt. Es folgten weitere Waschschritte: 1x5 min bei RT in 2xSSC, 1x30 min bei RT in 2xSSC/50 \% Formamid, 1x30 min bei $55^{\circ} \mathrm{C}$ in $2 \mathrm{xSSC} / 50 \%$ Formamid, $1 \mathrm{x} 15 \mathrm{~min}$ bei $55^{\circ} \mathrm{C}$ in $0,1 \mathrm{xSSC} / 50 \%$ Formamid, $2 \mathrm{x} 15$ min bei $55^{\circ} \mathrm{C}$ in $0,1 \mathrm{xSSC}$ sowie zuletzt auf dem Schüttler $2 \mathrm{x} 10 \mathrm{~min}$ bei RT in TBS $(0,05$ $\mathrm{M}, \mathrm{pH}$ 7,5). Vor der Detektierung der Sonden erfolgte unter ständigem Schütteln für eine Stunde die Blockierung mit 4\%igem Schafserum in TBS $(0,05 \mathrm{M}, \mathrm{pH} 7,5)$ bei RT. Danach wurden die Schnitte bei RT 3x2 min in TBS $(0,05 \mathrm{M}, \mathrm{pH} 7,5)$ gewaschen und anschließend bei RT 1x60 min in 0,5\% Blocking-Reagenz aus dem TSA Biotin Kit gegeben. Für die Detektion der Sonden wurden die Schnitte bei $4{ }^{\circ} \mathrm{C}$ über Nacht in Anti-Digoxigenin-POD (1:2000 in 0,5 $\%$ Blocking-Reagenz) inkubiert. 
Am dritten Tag wurden die Schnitte bei RT zuerst 3x10 min in TBS $(0,05 \mathrm{M}, \mathrm{pH} 7,5)$ gewaschen und dann für die Verbesserung der Visualisierung der Sonden für 3,5 Stunden bei RT in Biotinyl Tyramid (mind. $200 \mu \mathrm{l} /$ Schnitt/Well) biotinyliert. Nach anschließendem Waschen von 3x5 min und 1x15 min in TBS $(0,05 \mathrm{M}, \mathrm{pH} 7,5)$ erfolgte die Konjugation für 1x15 min mit AlexaFluor488gekoppeltem Streptavidin 1:400 (Life Technologies, Darmstadt, Deutschland) in TBS $(0,05 \mathrm{M}, \mathrm{pH} 7,5)$. Zuletzt wurden die Schnitte für 3x5 min und $1 \times 15 \mathrm{~min}$ in TBS $(0,05 \mathrm{M}$, $\mathrm{pH} 7,5)$ gewaschen.

Um das native PV/tdTomato-Signal zu amplifizieren, welches durch die Behandlungsschritte der FISH komplett ausgewaschen wurde, erfolgte im Anschluss eine immunhistochemische Färbung (Protokoll analog Abschnitt 2.2.1.), jedoch ohne Verwendung von Triton X-100. Alle nachfolgenden Arbeitsschritte mussten unter Dunkelbedingungen stattfinden.

\subsection{Auszählung / Auswertung}

Für die Auszählung wurden vier PVcre-Tiere mit jeweils zwei Schnitten pro Antikörper bzw. Sonde verwendet und in die Auswertung einbezogen. Um die behandelten Hirnschnitte visualisieren und auswerten zu können, wurde mit Hilfe eines Software-gesteuerten Mikroskops (AxioImager.M2, Zeiss, Jena, Deutschland)) mit dem Programm Neurolucida (MBF Bioscience, Williston, Vermont, USA) Bilder aufgenommen. Pro Färbung wurden je 2 Übersichtsaufnahmen des kompletten Hirnschnittes in einer 10fachen Vergrößerung in einer Ebene erstellt. Für die quantitative Analyse wurden dreidimensionale Bilder vom Bereich des Barrel-Kortex in 25facher Vergrößerung mithilfe eines ApoTomes (Zeiss, Jena, Deutschland) angefertigt. Die Aufnahme umfasste über mehrere Kacheln den Barrel-Kortex von der Pia mater bis zur weißen Substanz sowie mindestens 3 Barrel-assoziierte Kolumnen. In der z-Ebene wurde eine Auflösung von einem Mikrometer eingestellt, d. h. alle $1 \mu \mathrm{m}$ ein Bild aufgenommen. Die weitere Analyse erfolgte ebenfalls mit dem Programm Neurolucida. In den aufgenommen und durch Neurolucida zusammengesetzten Kacheln wurde ein $1000 \mu \mathrm{m}$ breiten Bereich für die Analyse ausgewählt und mit einer Kontur festgehalten. Einzelne Zellen, die mit ihrem Soma auf der Kontur lagen, wurden bei einer Lage von mehr als $50 \%$ innerhalb der Kontur in die Analyse einbezogen. Die sich in dem umrandeten Bereich befindenden Zellkerne wurden jeweils ihrer Färbung nach gesondert per Hand gezählt und markiert.

Nach der manuellen Zellzählung wurden mit Hilfe des Programms innerhalb des markierten Bereichs die Schichten (Laminae) eingezeichnet. Dafür konnte die charakteristische Zelldichte 
der einzelnen kortikalen Schichten, die durch die Zellkernfärbung mittels DAPI visualisiert wurden, herangezogen werden (siehe 1.1).

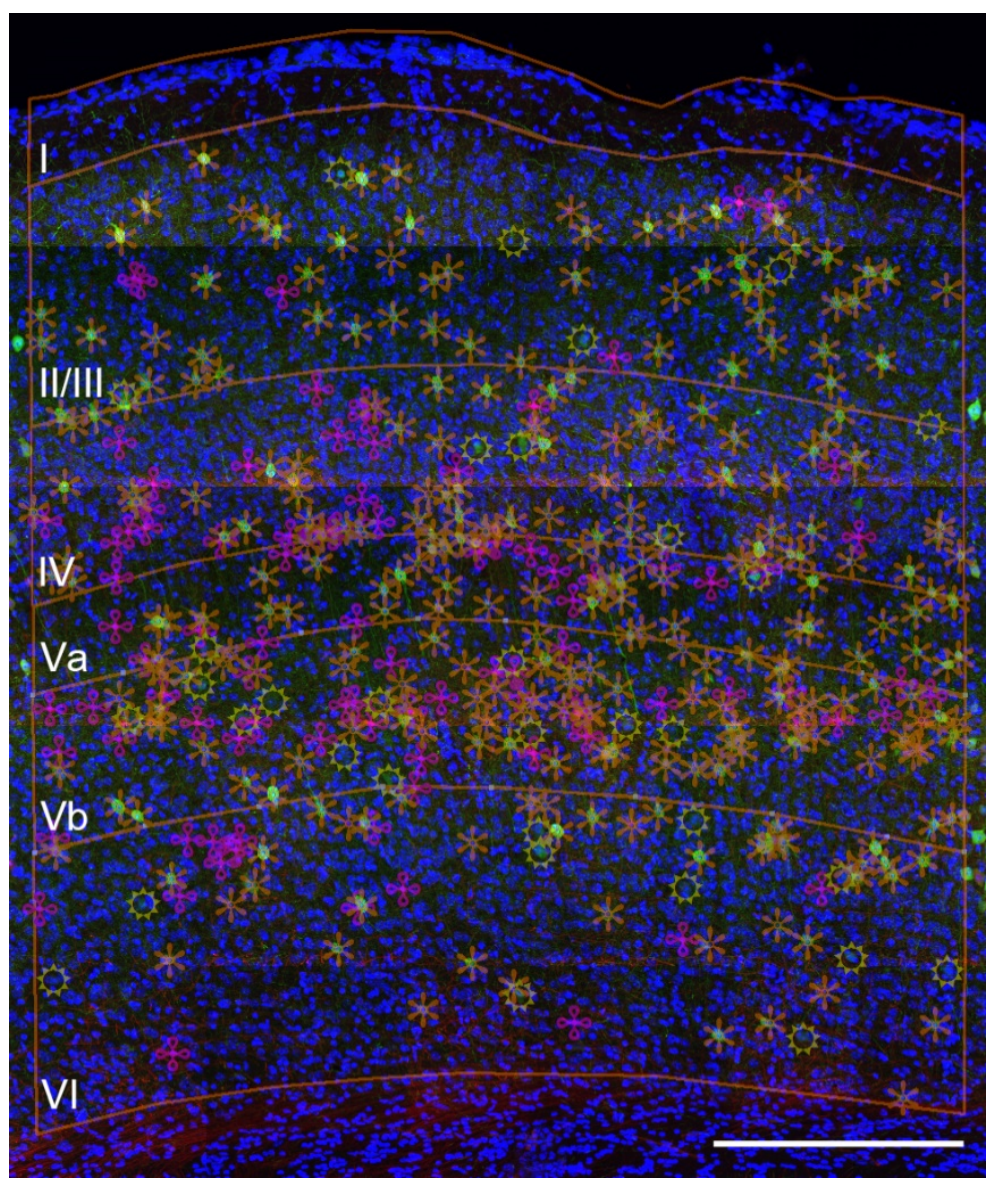

Abbildung 2.1: SchichtenAnalyse

PV-Antikörper Immunhistochemie an einem frontalen Hirnschnitt einer PVcre/tdTomato-Maus zeigt in den verschiedenen Schichten IVI in Rot das transgene tdTomatoSignal, in Grün die $\alpha$ PV-positiven Zellen und die Zellkerne (DAPI) in Blau. In dem ausgewählten Bereich sind die unterschiedlichen Symbole in den neokortikalen Schichten voneinander abgegrenzt; Maßstab $250 \mu \mathrm{m}$

Zu Beginn konnte die Schicht IV durch die gut erkennbare Struktur der Barrel eingezeichnet werden. Der umrandete Bereich verdeutlicht einen zelldichten Streifen.

Zwischen der Pia mater und der zellreicheren Schicht II/III kann die zellarme Schicht I gut abgegrenzt werden. Dabei wurde die Pia mater, die teilweise bei einigen Schnitten unvollständig war, mit in die Schicht I einbezogen. Nachdem die Schichten I und IV abgegrenzt wurden, konnte die dazwischen befindende Schicht II/III klar festgelegen werden. Dabei wurde diese Schicht aufgrund der nicht klaren Differenzierung von Schicht II und III als ein gemeinsamer Komplex zusammengefasst. Die Schicht V lässt sich wie folgt in Va und Vb gliedern. Einerseits besitzt Schicht Va eine geringere Zelldichte als Schicht Vb und andererseits macht Va circa ein Drittel der Schichtdicke der Schicht V aus (Schubert et al. 2006). Die Schicht Vb kann von der darauffolgenden Schicht VI klar durch die erhöhte Zelldichte in VI differenziert werden. Als untere Begrenzung der Schicht VI wurde der Beginn der Zellkerne von Gliazellen in der weißen 
Substanz genutzt. Über die Software Neuroexplorer (MBF Bioscience, Williston, Vermont, USA) konnte letztlich eine schichtspezifische Auszählung durchgeführt. Das Programm gab Auskunft über die Zahlen der einzelnen Zellmarker und die Fläche in den einzelnen Schichten sowie für den gesamten ausgezählten Bereich des Barrel-Kortex. Anhand dieser Informationen konnte eine statistische Auswertung der Ergebnisse durchgeführt werden. 


\section{Ergebnisse}

Das Ziel dieser Studie ist die schichtenspezifische Charakterisierung Parvalbuminexprimierender Neurone in der PVcre/tdTomato-Maus im Bereich des somatosensorischen Kortex (Barrel-Kortex) mithilfe der Immunhistochemie (IHC) und der Fluoreszenz-in-situHybridisierung (FISH). In diesem Zusammenhang soll die Spezifität sowie Sensitivität der tdTomato-positiven Zellen in der PVcre/tdTomato-Maus für die PV-Expression untersucht werden. Zusätzlich werden Untersuchungen anhand der FISH über eine potenzielle Kolokalisation mit den GABAergen Interneuronmarkern Somatostatin (SOM) und vasoaktives intestinales Polypeptid (VIP) durchgeführt. Durch Verwendung eines inhibitorischen, Glutamatdecarboxylase 1 (Gad1), und eines exzitatorischen, vesikulären Glutamattransporter 1 (Vglut1), Markers, können Rückschlüsse über hemmende oder erregende Eigenschaften der tdTomatopositiven Zellen der PVcre/tdTomato-Maus getroffen werden. Um weitere Erkenntnisse über die PV-exprimierenden Interneurone zu erlangen, wurden die perineuronalen Netze in der extrazellulären Matrix mittels IHC angefärbt und deren räumliche Nähe zu den tdTomatopositiven Zellen betrachtet.

\subsection{Sind die tdTomato-positiven Zellen in der PVcre-Maus auch Parvalbumin-positiv $(+)$ ?}

Die in dieser Studie eingesetzte PVcre/tdTomato-Maus weist durch die Verwendung des Cre/loxP-Systems eine native rote Fluoreszenz in PV-exprimierenden Neuronen auf. Die Ergebnisse meiner Arbeit sollen zur Klärung der Frage beitragen, ob die tdTomato-positiven (+) Zellen in der PVcre-Maus auch Parvalbumin-positiv (+) sind. Des Weiteren wurde die schichtenspezifische Verteilung der tdTomato-positiven Zellen betrachtet und analysiert. Für den Nachweis, ob es sich tatsächlich um rot fluoreszierende PV-exprimierende Zellen handelt, wurden sieben Schnitte von vier verschiedenen Tieren (zwei Schnitte pro Tier) untersucht. Sie wurden mit dem PV-Antikörper der Firma Swant behandelt und digitale Aufnahmen zur Auswertung angefertigt. 

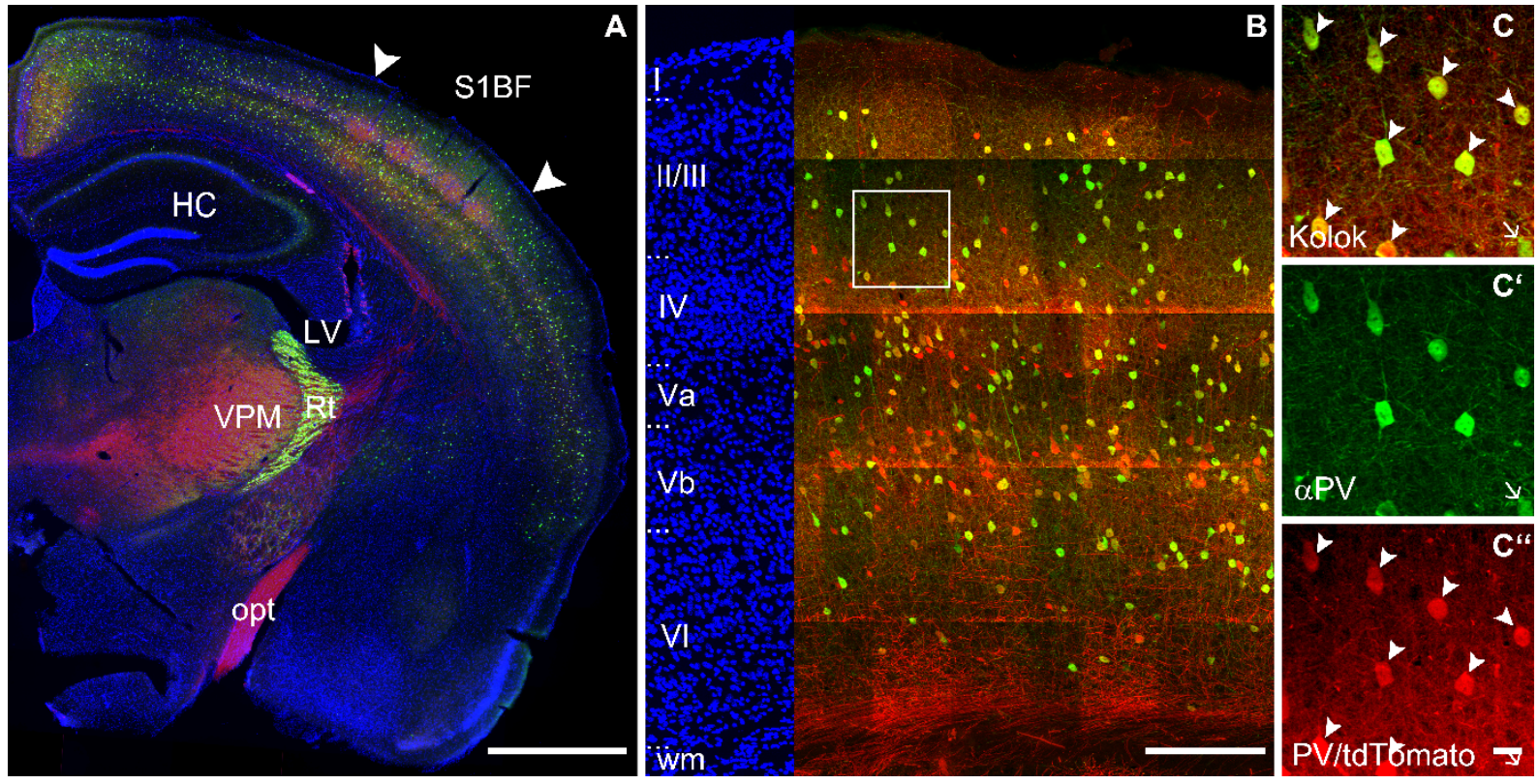

Abbildung 3.1: Ausschnitte einer PV-Antikörper-Färbung zur Darstellung PV-positiver Zellen im Bereich des Barrel-Kortex der PVcre/tdTomato-Maus

Immunhistochemie mittels PV-Antikörper an frontalen Hirnschnitten einer PVcre/tdTomato-Maus, bei der in Rot das transgene tdTomato-Signal, in Grün der aPV-Antikörper (Swant), in Blau die Zellkerne (DAPI) und in Gelb die Kolokalisation zu erkennen sind. In $\mathbf{A}$ ist eine optische Einzelebene in der 10fachen Vergrößerung der linken Hemisphäre im Bereich des Barrel-Kortex (Pfeilspitzen; S1BF) dargestellt; Maßstab $1000 \mu \mathrm{m}$. Des Weiteren sind die Strukturen Hippocampus (HC), lateraler Ventrikel (LV), Tractus opticus (opt) und die Thalamuskerne, Nucleus ventralis posteromedialis (VPM) und Nucleus reticularis (Rt), zu sehen. (B) In der maximum intensity projection des Barrel-Kortex in der 25 fachen Vergrößerung sind die neokortikalen Schichten durch römische Zahlen angegeben; Maßstab $200 \mu \mathrm{m}$. Der markierte Bereich wird in C-C" vergrößert wiedergegeben; Maßstab $20 \mu \mathrm{m}$. C zeigt die Kolokalisation des tdTomato und des PV-Antikörper Signals in Gelb, $\mathbf{C}^{\prime}$ zeigt die mit dem PV-AK markierten Zellen und $\mathbf{C}^{\prime \prime}$ das native tdTomato-Signal. Die Pfeilspitzen markieren die mit dem PVAntikörper kolokalisierten tdTomato-positiven Zellen, wohingegen die Pfeile auf ausschließlich mit dem Antikörper markierte Zelle verweist (C-C').

Der PV-Antikörper zeigt in der Übersichtsaufnahme eines frontalen Hirnschnittes eine deutliche Anfärbung von Somata im Bereich des Neokortex, im Nucleus retikularis (Rt) des Thalamus und vereinzelte Zellen im Hippocampus (Abb. 3.1A). Im Bereich des Barrel-Kortex (S1BF) ist die Darstellung der Barrels innerhalb der Schicht IV durch die native tdTomatoFluoreszenz klar zu erkennen. Ebenfalls tritt der Nucleus ventralis posteromedialis (VPM) sowie die Fasern des Tractus opticus durch die rote Fluoreszenz hervor. Die tdTomato markierten Zellen im S1BF wurden im Folgenden, in Hinblick auf die schichtenspezifische Lage und deren Kolokalisation mit dem PV-Antikörper untersucht. In der 25fachen Vergrößerung kann man deutlich das vermehrte Auftreten der tdTomato und PV-positiven Zellen in Schicht IV sowie $\mathrm{Vb}$ erkennen (Abb. 3.1B). Dabei kommen beide Signale überwiegend überlagert vor, $\mathrm{d}$. $\mathrm{h}$. kolokalisiert, welche durch eine Gelbfärbung sichtbar wird. Die Bestätigung der Kolokalisation 
erlangt man durch die Auftrennung der Signale des PV-Antikörper und der nativen tdTomatoFluoreszenz (Abb. 3.1C). Weiterhin wird das fehlende Vorkommen der PV-Zellen in der Schicht I verdeutlicht (Abb. 3.1B).

Die schichtspezifische Verteilung der kolokalisierten PV-positiven/tdTomato-positivenZellen, der nur PV-positiven und der nur tdTomato-positiven Zellen wurde auf der Basis von sieben ausgezählten Hirnschnitten erstellt (Abb. 3.2). Innerhalb eines definierten $1000 \mu \mathrm{m}$ breiten Abschnitts des Barrel-Kortex weisen im Mittel 8943,5 \pm 383,6 Zellen pro mm³ BarrelKortex (extrapoliert) eine native tdTomato-Fluoreszenz auf (Tab. 6.1). Davon sind 7846,6 \pm 816,0 Zellen mit dem PV-Antikörper kolokalisiert und eine große Anzahl von 1097,0 \pm 561,8 tdTomato-Zellen tragen kein PV-Antikörper Signal (Abb.3.2). Demzufolge sind 87,6 \pm 6,7 \% der tdTomato-Zellen mit dem PV-Antikörper im Bereich des Barrel-Kortex kolokalisiert. In fünf von sieben Hirnschnitten wurden einige nur aPV positive Zellen registriert und machten im Mittel 348,4 \pm 279,2 Zellen pro mm³ $^{3}$ Barrel-Kortex aus (Abb.3.2). 


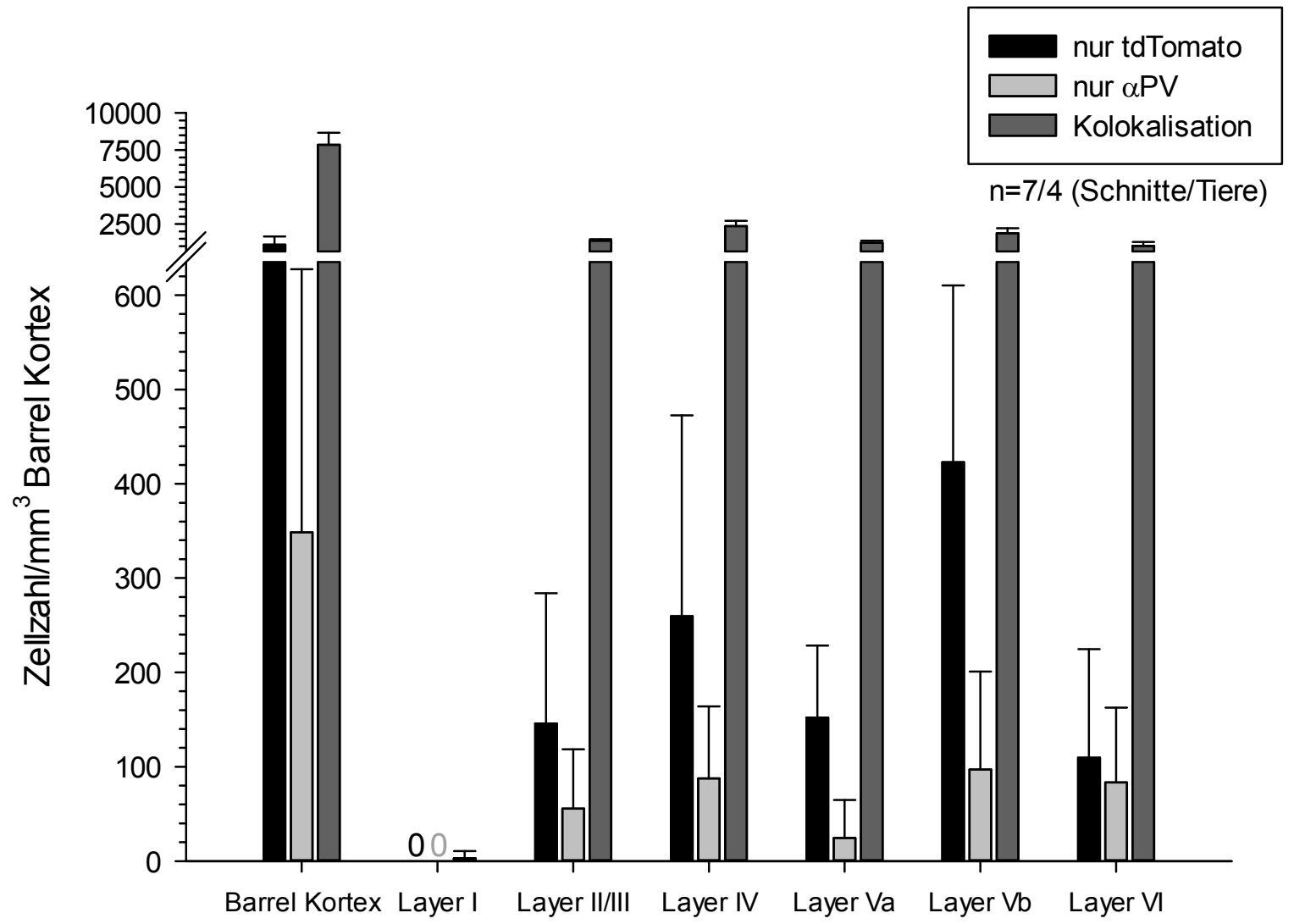

\section{Abbildung 3.2: Kolokalisation von PVcre/tdTomato-Zellen und dem PV-Antikörper}

In dem Balkendiagramm sind die Anzahl der Kolokalisationen zwischen den PV/tdTomato-Zellen und dem PV-Antikörper der Firma Swant in Bezug auf die einzelnen Schichten des Barrel-Kortex dargestellt (grauer Balken). Außerdem wird die Anzahl der nur PV/tdTomato-Zellen (schwarzer Balken) und der nur PV-Antikörper tragender Zellen (hellgrauer Balken) angegeben. Nicht kolokalisierte Zellen kommen in allen kortikalen Schichten in deutlich geringer Zahl als die kolokalisierten vor. Die angegebenen Werte sind bezogen auf $1 \mathrm{~mm}^{3}$ Barrel-Kortex und für die Schichten I-VI.

Für die einzelnen kortikalen Schichten lässt sich festhalten, dass in allen deutlich mehr kolokalisierte Zellen vorkommen als nicht-kolokalisierte. Dabei ist die Anzahl mit 2357,7 \pm 364,9 kolokalisierten Zellen in der Schicht IV am größten, gefolgt von der Schicht Vb mit 1874,8 \pm 336,2 Zellen, der Schicht II/III mit 1368,8 \pm 107,8 und der Schicht Va mit 1230,9 \pm 139,3 Zellen. In der Schicht I konnten lediglich in einem von sieben Hirnschnitten kolokalisierte Zellen beobachtet werden. Dagegen war in Schicht VI in allen sieben Hirnschnitten markierte Zellen zu finden, wobei deren Anzahl mit 1014,6 \pm 276,8 kolokalisierten Zellen am geringsten ausfällt. Nicht-kolokalisierte Zellen zeigen eine ähnliche Verteilung, d. h., dass die Mehrzahl in den Schichten IV mit 259,6 \pm 213,1 Zellen und vor allem in Schicht Vb mit 423,0 \pm 187,3 Zellen lokalisiert sind. Ausschließlich tdTomato-positive Zellen sind in allen Schichten häufiger anzutreffen als Zellen, die nur das PV-Antikörper Signal aufweisen. 
Um die schichtenspezifische Verteilung der rot fluoreszierenden PV/tdTomato-Zellen im Barrel-Kortex eingehender zu untersuchen, wurden die Zellzahlen von allen 47 ausgewerteten Schnitten der unterschiedlichen Färbungen zusammengetragen und analysiert (Abb. 3.3). Die Schichtenabgrenzung erfolgte wie in Abschnitt 2.4 beschrieben und wurde mehrmals überprüft. Für die PVcre/tdTomato-Maus lässt sich festhalten, dass der größte Anteil der tdTomatopositiven Zellen in der Schicht IV mit 2857,6 \pm 279,6 Zellen vorkommt. Das entspricht einem Anteil von 28,7 $\pm 2,1 \%$ aller tdTomato-Zellen. In sieben von 47 Schnitten konnten mehr Zellen in der Schicht Vb beobachtet werden, wobei deren Anzahl im Mittel mit 2468,8 \pm 403,7 Zellen und einem Anteil von 24,7 \pm 2,9\% knapp hinter der Schicht IV liegt. Berücksichtigt man dazu noch den Anteil der tdTomato-Zellen in der Schicht Va mit 1499,8 \pm 220,8 Zellen und einem relativen Anteil von 25,1 \pm 2,2\%, dann entfallen fast $40 \%$ der tdTomato-Zellen auf die Schicht V. Demzufolge liegt der Anteil der tdTomato-Zellen in den Schichten I, II/III und VI bei etwas über $30 \%$ und teilt sich wie folgt auf. Die Schichten II/III und VI weisen 1617,3 \pm 207,7 Zellen und 16,3 $\pm 2,2 \%$ bzw. 1522,8 \pm 349,4 Zellen und 15,1 $\pm 2,8 \%$ an tdTomato-positiven Zellen auf. In der Schicht I konnten lediglich 5,6 \pm 12,5 Zellen detektiert werden, das entspricht einen Anteil der tdTomato-Zellen von insgesamt $0,1 \pm 0,1 \%$ und beruht wahrscheinlich auf ihrer Grenzlage zur Schicht II/III, zu der sie zugehörig sein sollten. Des Weiteren konnten lediglich in neun von 47 Schnitten PV/tdTomato-Zellen in der tiefen Schicht I beobachtet werden. 

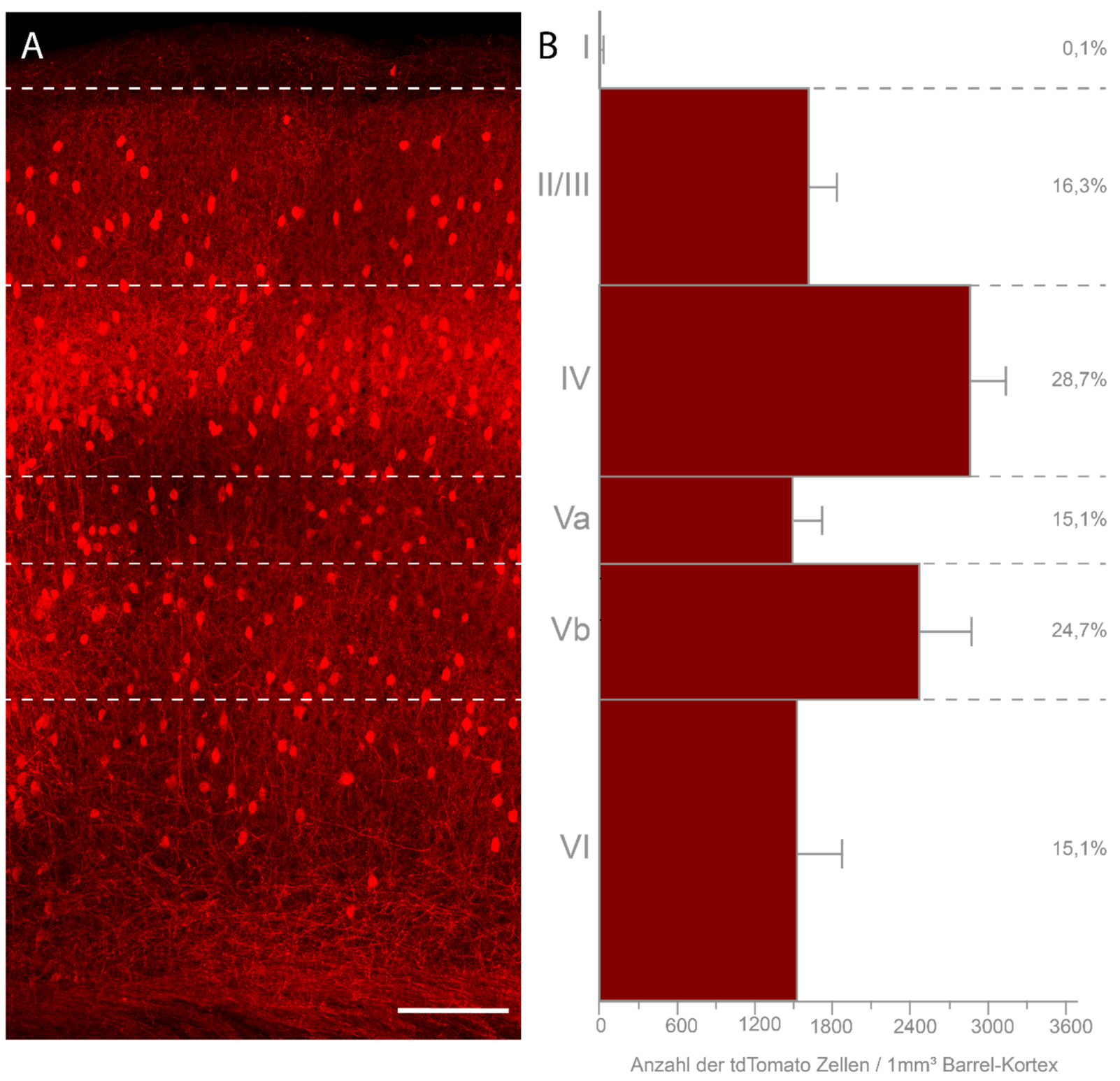

Abbildung 3.3: Schichtenspezifische Verteilung der tdTomato-Zellen im Barrel-Kortex der PVcre/tdTomato-Maus

(A) Epifluoreszenzaufnahme eines $300 \mu \mathrm{m}$ dicken Frontalschnittes einer PVcre/tdTomato-Maus im Bereich des Barrel-Kortex und der nativen rot fluoreszierenden PVcre-Zellen, Maßstab $125 \mu \mathrm{m}$. (B) Schichtenspezifische Verteilung der tdTomato-Zellen in 47 untersuchten Hirnschnitten extrapoliert auf $1 \mathrm{~mm}^{3}$ Barrel-Kortex. Römischen Zahlen (links) repräsentieren die einzelnen neokortikalen Schichten und die prozentualen Angaben (rechts) spiegeln die relative Verteilung über die Schichten wider.

Zusammenfassend zeigt sich mit über $68 \%$ eine ausgeprägte fokussierte Lage der tdTomatoZellen in den Schichten IV und V. Dies könnte auf ein spezialisiertes Aufgabenprofil der PVZellen im Bereich des Barrel-Kortex hindeuten. Des Weiteren besteht ein hoher Anteil von ca. $87 \%$ an Kolokalisation zwischen den tdTomato-Zellen und dem PV-Antikörper. Jedoch fällt der Anteil der nicht mit dem PV-Antikörper kolokalisierten Zellen insbesondere in den 
Schichten IV und V am höchsten aus. Daher stellt sich die Frage, was für Zellen in diesen Schichten mit dem tdTomato-Signal markiert sind.

\subsection{Sind die tdTomato-positiven Zellen im Barrel Kortex exzitatorisch oder inhibitorisch?}

Zu den drei Hauptgruppen der GABAergen Interneurone, die als inhibitorische Zellen bekannt sind, gehört als größte Population die der Parvalbumin-exprimierenden Interneurone. Allerdings exprimieren nicht nur inhibitorische Interneurone das kalziumbindende Protein Parvalbumin. Es wurde bereits vor 25 Jahren in einer weitgehend ignorierten Arbeit gezeigt, dass insbesondere Zellen im somatosensorischen Kortex der Ratte mit vertikal orientierten primären Dendriten und pyramidalem Soma immunpositiv für PV sind (van Brederode et al. 1991). Deswegen soll hier untersucht werden, inwiefern die tdTomato-positiven Zellen der PVcre-Maus exzitatorische bzw. inhibitorische Eigenschaften aufweisen und wie deren Verteilung in den einzelnen kortikalen Schichten ausfällt. Zur Markierung der tdTomato-Zellen wurden die Sonden Gad1 und Vglut1 in der Versuchsreihe der FISH verwendet. Insgesamt wurden pro eingesetzte Sonde zwei Hirnschnitte von vier verschiedenen Tieren (acht Schnitte pro Sonde) ausgewertet.

Die Gad1-Sonde markiert die Glutamatdecarboxylase 1 (Gad67) und identifiziert inhibitorische Zellen. Die Glutamatdecarboxylase katalysiert die Umwandlung von Glutamat zu GABA, das von inhibitorischen Neuronen ausgeschüttet wird. Im Gegensatz dazu wird dem vesikulären Glutamattransporter 1 die Aufgabe zugeschrieben, den exzitatorsichen Neurotransmitter Glutamat in Vesikel zu verpacken, um eine Freisetzung in den synaptischen Spalt zu gewährleisten. Vglut1 kann aus diesem Grund als Marker exzitatorischer Zellen eingesetzt werden. 
Die Abbildung eines frontalen Hirnschnittes in der Übersichtsaufnahme der Gad1-FISH zeigt eine starke Markierung im Bereich des Nucleus reticularis (Rt), einiger Somata im gesamten Neokortex und vereinzelter Zellen im Hippocampus (Abb. 3.4A). Der durch die Pfeile begrenzte Bereich des Barrel-Kortex (S1BF) wird aufgrund der Barrels, die sich in Schicht IV durch das stärkere native tdTomato-Signal hervorheben, verdeutlicht. Die Gad1-positiven Somata (Gad67) zeigen eine mehr oder weniger gleichmäßige Verteilung über alle neokortikalen Schichten hinweg. Dagegen beschränkt sich die rote Fluoreszenz der nativen tdTomato-Zellen auf die mittleren neokortikalen Schichten. Im Hinblick auf die Fragestellung, ob die tdTomatomarkierten Zellen im S1BF inhibitorische Merkmale aufweisen, wurden sie auf mögliche Kolokalisation mit den Gad1-markierten Zellen überprüft und ihre schichtspezifische Lage analysiert.
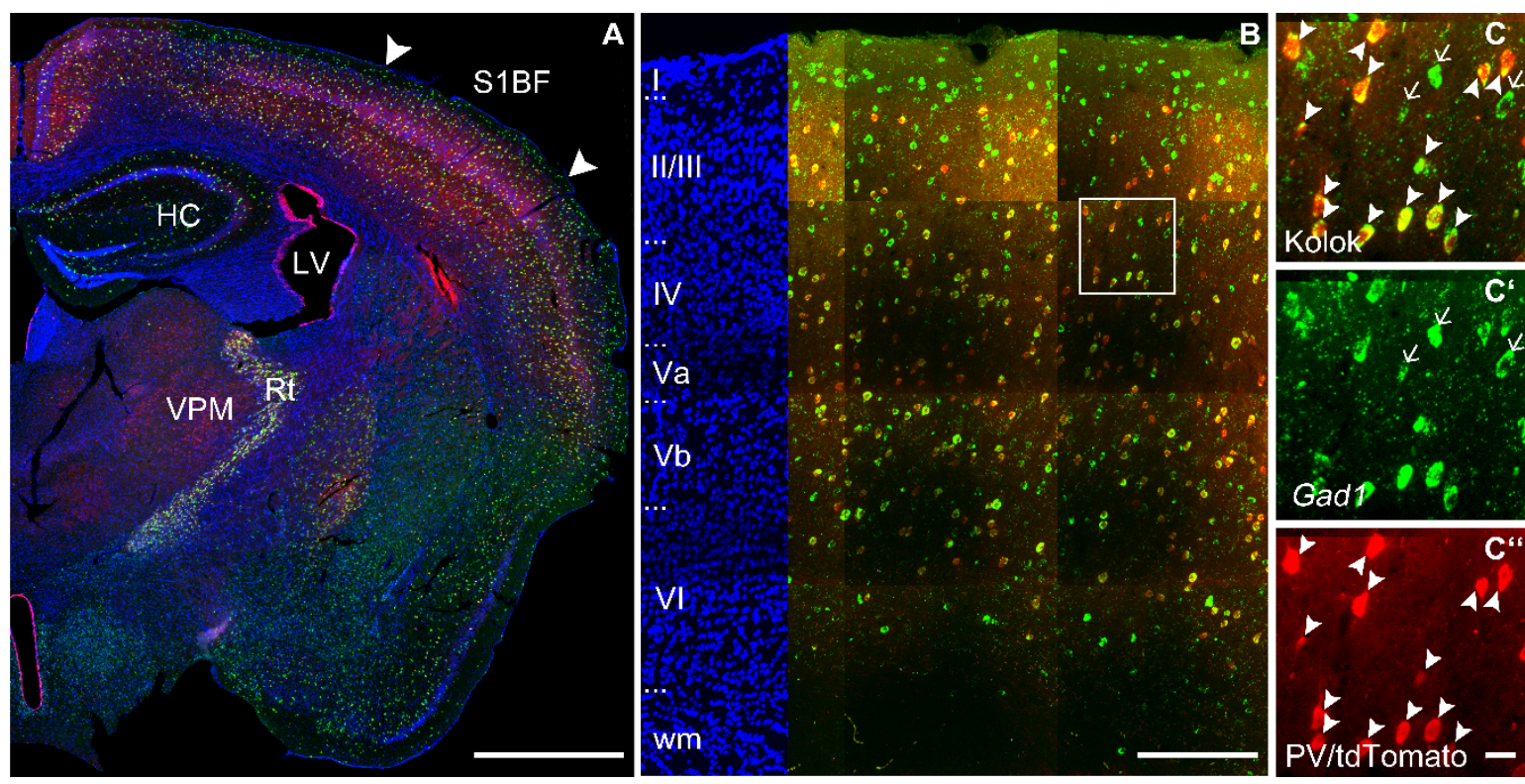

Abbildung 3.4: FISH zur Darstellung Gad1-positiver Zellen im Bereich des Barrel-Kortex der PVcre/tdTomato-Maus

Fluoreszenz-in-situ-Hybridisierung mittels Gad1-Sonde (Gad67) an frontalen Hirnschnitten einer PVcre/tdTomato-Maus, bei der in Rot das transgene tdTomato-Signal, in Grün die Gad1-positiven Zellen, in Blau die Zellkerne (DAPI) und in Gelb die Kolokalisation zu erkennen sind. In $\mathbf{A}$ ist eine Einzelebene in der 10fachen Vergrößerung der linken Hemisphäre im Bereich des Barrel-Kortex (Pfeilspitzen; S1BF) dargestellt; Maßstab $1000 \mu \mathrm{m}$. Des Weiteren sind die Strukturen Hippocampus (HC), lateraler Ventrikel (LV) und die Thalamuskerne, Nucleus ventralis posteromedialis (VPM) und Nucleus reticularis (Rt), gekennzeichnet. (B) In der maximum intensity projection des Barrel-Kortex in der 25fachen Vergrößerung sind die neokortikalen Schichten durch römische Zahlen angegeben; Maßstab $200 \mu \mathrm{m}$. Der markierte Bereich wird in C-C' vergrößert wiedergegeben; Maßstab $20 \mu \mathrm{m}$. C zeigt die Kolokalisation des tdTomato und des Gad1-Sonde Signals in Gelb, $\mathbf{C}^{\prime}$ zeigt die mit der Gad1-Sonde (Gad67) markierten Zellen und $\mathbf{C}^{\prime \prime}$ das native tdTomato-Signal. Die Pfeilspitzen markieren die mit der Gad1-Sonde kolokalisierten tdTomato-positiven Zellen, wohingegen die Pfeile auf ausschließlich mit der Sonde markierte Zellen verweisen (C-C' $\mathbf{C}^{\prime \prime}$ ). 
In der 25fachen Vergrößerung wird die häufige Überlagerung der beiden Signale deutlich (Abb. 3.4B). Ferner lassen sich auch grün fluoreszierende Somata ohne Kolokalisation innerhalb der neokortikalen Schichten finden. Bei genauerer Betrachtung erkennt man in Schicht IV sowie in Schicht V einzelne ausschließlich rot fluoreszierende native tdTomato-Zellen. Die Vergrößerung des in Schicht II/III und IV gelegenen Rechtecks zeigt die Kolokalisation sowie die Auftrennung der beiden Signale (Abb. 3.4C). In $C^{\prime}$ verdeutlichen die Pfeile, die auch in C zu finden sind, Gad1-markierte Zellen ohne Überlagerung. Diese Somata sind in C"' nicht mehr vorhanden und tragen folglich kein natives tdTomato.

Die Pfeilspitzen in den detaillierten Ausschnitten C und C" veranschaulichen die Kolokalisation der Gad1-Sonde und des nativen tdTomato-Signals. In C" sind die Somata der tdTomato-Zellen markiert; in C spiegeln die Pfeilspitzen die exakte Position der Somata mit dem überlagerten Grünkanal der Gad1-Sonde wider. Der gegenüberstellende Vergleich der kolokalisierten tdTomato-Zellen mit der Gad1-Sonde und den nur tdTomato-tragenden Zellen erfolgte schichtspezifisch anhand acht ausgezählter Hirnschnitten (Abb. 3.5). Nach der quantitativen Auswertung eines $1000 \mu \mathrm{m}$ breiten Ausschnittes können folgende Aussagen getroffen werden: In einem $1 \mathrm{~mm}^{3}$ großen Bereich des Barrel-Kortex finden sich im Mittel 10540,7 \pm 822,4 Zellen mit nativer tdTomato-Fluoreszenz (Tab. 6.5). Von diesen weisen 9701,4 \pm 807,2 tdTomatopositive Zellen eine Kolokalisation mit der Gad1-Sonde auf und lediglich 839,3 \pm 342,4 Zellen besitzen keine Signalüberlagerung (Abb. 3.5). Folglich sind 92,1 \pm 3,3\% aller tdTomato-Zellen Gad1-positiv und können als inhibitorische Neurone angesehen werden (Abb. 3.8, Tab. 6.7). 


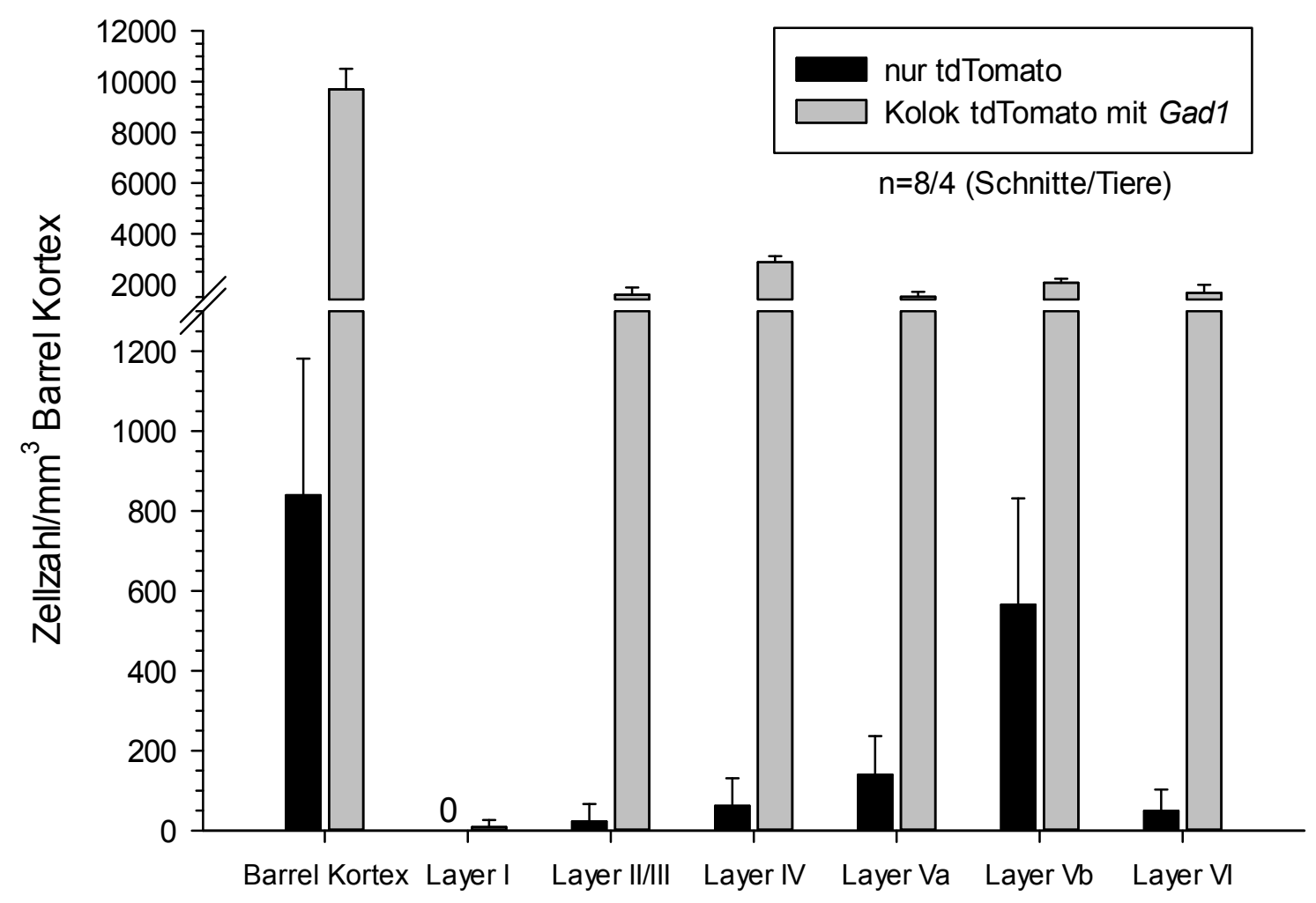

Abbildung 3.5: Kolokalisation zwischen den tdTomato-Zellen und der Gad1-Sonde

Das Balkendiagramm präsentiert die Anzahl der Kolokalisationen zwischen den tdTomato-Zellen der PVcre/tdTomato-Maus und der Gad1-Sonde (grau) sowie die nicht kolokalisierten tdTomato-Zellen (schwary). Die angegebenen Werte sind bezogen auf $1 \mathrm{~mm}^{3}$ Barrel-Kortex und für die Schichten I-VI.

Aus dem Diagramm wird deutlich, dass innerhalb der kortikalen Schichten der größte Anteil der tdTomato-Zellen eine Kolokalisation mit der Gad1-Sonde aufweist (Abb. 3.5). Von ihnen befinden sich die meisten in Schicht IV mit 2870,9 \pm 246,7 Zellen, gefolgt von der Schicht Vb mit 2062,2 \pm 163,1 kolokalisierten Zellen. Hingegen wird in Schicht II/III 1591,9 \pm 287,1, in Schicht Va 1515,7 \pm 189,2 und in Schicht VI nur 1660,8 \pm 324,3 einen annähernd hohen Präsenz an Kolokalisation gefunden. Lediglich in zwei von acht Hirnschnitten werden 8,8 \pm 17,3 kolokalisierte Zellen in Schicht I gefunden. Der geringe Anteil an Zellen, die nicht durch die Gad1-Sonde markiert wurden, sticht in Schicht Va mit 139,7 \pm 96,9 und ganz markant in Schicht Vb mit 565,4 \pm 265,8 Zellen hervor (Abb. 3.5, Tab. 6.5). Dies zeigt sich auch bei der prozentualen Betrachtung der Kolokalisation der tdTomato-Zellen, die Gad1-positiv sind. Bis auf Schicht Va mit 91,6 \pm 5,7 \% und Schicht $\mathrm{Vb}$ mit 79,3 \pm 7,9 \% liegt die Kolokalisation der restlichen Schichten bei über 97 \% (Abb. 3.8, Tab.6.7). In den Schichten II/III-VI werden nicht in allen ausgezählten Hirnschnitten tdTomato-Zellen ohne Gad1 Kolokalisation gefunden: 
Schicht II/III in drei von acht Schnitten mit 22,8 \pm 43,7 Zellen, Schicht IV in fünf von acht Schnitten mit 62,1 \pm 68,7 und Schicht VI in sieben von acht Schnitten mit 49,3 \pm 53,2 Zellen. In Schicht I befinden sich keine nicht-kolokalisierten tdTomato-Zellen.

Die Gegenprüfung, wie viele tdTomato-Zellen eine Expression eines exzitatorischen Markers aufweisen, erfolgt mittels der Vglut1-Sonde. In der Übersichtsaufnahme eines frontalen Hirnschnittes erkennt man, im Vergleich zu der Aufnahme der zu Beginn beschriebenen Gad1Sonde, die zahlenmäßige Dominanz der exzitatorischen Zellpopulation innerhalb der Neokortex (Abb. 3.6). Die grün fluoreszierenden Vglut1-positiven Zellen zeigen innerhalb der Schichten des gesamten Hirnschnittes eine dem DAPI-Signal analoge Verteilung. In der zellarmen Schicht I sind keine Vglut1-positiven Zellen zu finden. Des Weiteren treten in dieser Färbung die zelldichte Schicht IV sowie die großen Somata der exzitatorischen Zellen in der Schicht $\mathrm{V}$ hervor. Im Barrel-Kortex (S1BF) hebt sich zudem die native tdTomato-Fluoreszenz der Tönnchen innerhalb der Schicht IV markant hervor. Der VPM wird ebenfalls durch die rote Fluoreszenz verdeutlicht.

Die 25fache Vergrößerung veranschaulicht das hohe Vorkommen der Vglut1-positiven Zellen im Barrel-Kortex (Abb. 3.6B). Schon in dieser Vergrößerung erkennt man, dass die Somata der nativen tdTomato-Zellen separat auftreten und die Schicht I keine markierten Zellen besitzt. In dem detaillierten Ausschnitt aus Schicht II/III und IV sind die rot fluoreszierenden Somata mit Pfeilspitzen markiert (Abb. 3.6C-C') . Dabei ist keine Kolokalisation der tdTomato-positiven Zellen der PVcre-Maus mit der Vglut1-Sonde zu erkennen. Bei einer genaueren Betrachtung der infragranulären Schichten, $\mathrm{Va}$ sowie $\mathrm{Vb}$, kann man einzelne kolokalisierte Zellen entdecken (Abb. 3.6B). 

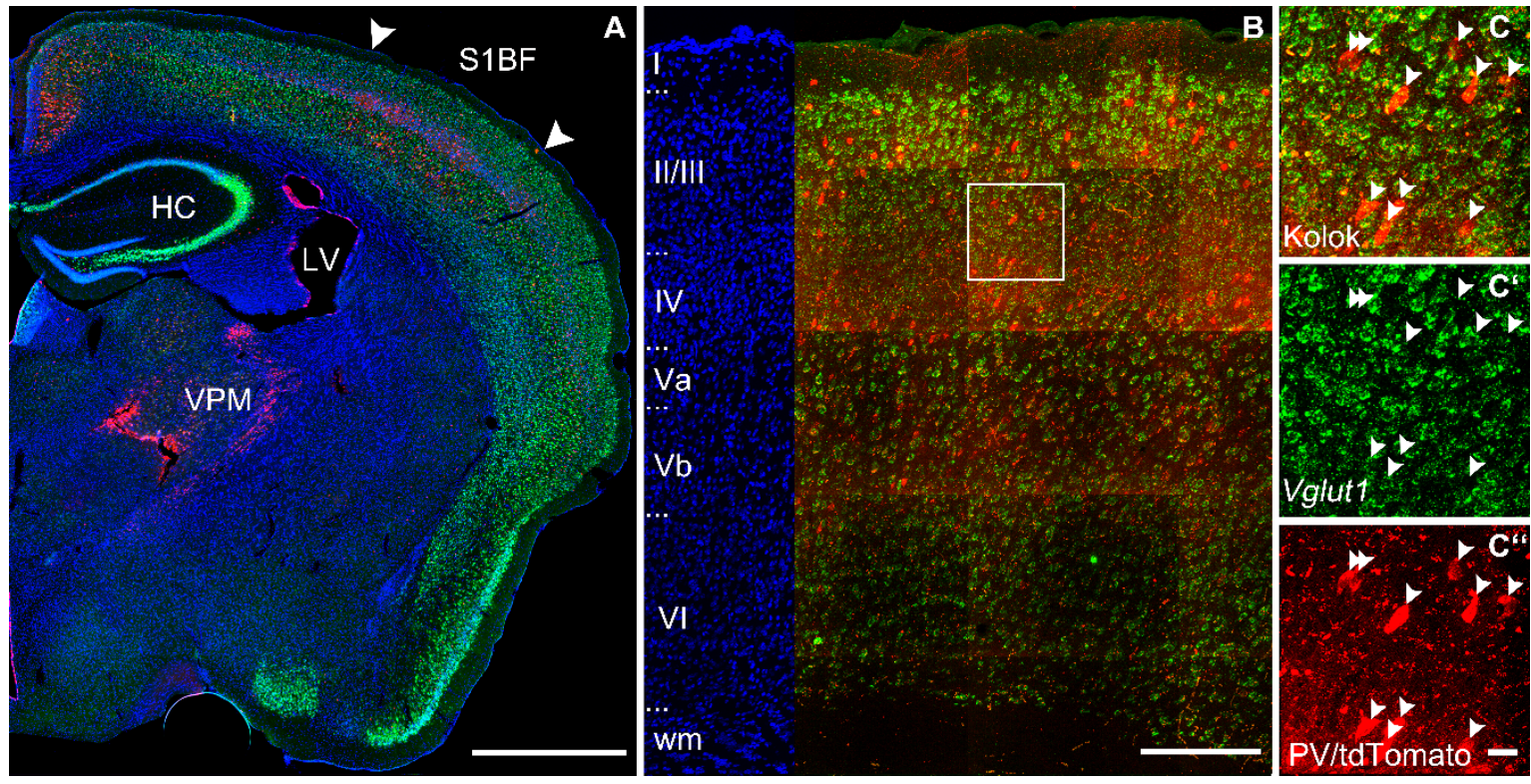

\section{Abbildung 3.6: FISH zur Darstellung Vglut1-positiver Zellen im Bereich des Barrel-Kortex der} PVcre/tdTomato-Maus

Fluoreszenz-in-situ-Hybridisierung mittels Vglut1-Sonde an frontalen Hirnschnitten einer PVcre/tdTomato-Maus, bei der in Rot das transgene tdTomato-Signal, in Grün die Vglut1-positiven Zellen, in Blau die Zellkerne (DAPI) und in Gelb die Kolokalisation zu erkennen sind. In A ist eine Einzelebene in der 10fachen Vergrößerung der linken Hemisphäre im Bereich des Barrel-Kortex (Pfeilspitzen; S1BF) dargestellt; Maßstab $1000 \mu \mathrm{m}$. Des Weiteren sind die Strukturen Hippocampus (HC), lateraler Ventrikel (LV) und der Thalamuskern Nucleus ventralis posteromedialis (VPM), zu sehen. (B) In der maximum intensity projection des Barrel-Kortex in der 25fachen Vergrößerung sind die neokortikalen Schichten durch römische Zahlen angegeben; Maßstab $200 \mu \mathrm{m}$. Der markierte Bereich aus dem Übergang von Schicht II/III zu IV wird in C-C" vergrößert wiedergegeben; Maßstab $20 \mu \mathrm{m}$. C zeigt die nicht vorhandene Kolokalisation des tdTomato und des Vglut1-Sonde Signals in Gelb, $\mathbf{C}^{\prime}$ zeigt die mit der Vglut1-Sonde markierten Zellen und $\mathbf{C}^{\prime \prime}$ das native tdTomato-Signal. Die Pfeilspitzen markieren die nicht kolokalisierten tdTomato-positiven Zellen im ausgewählten Ausschnitt (C-C'). 


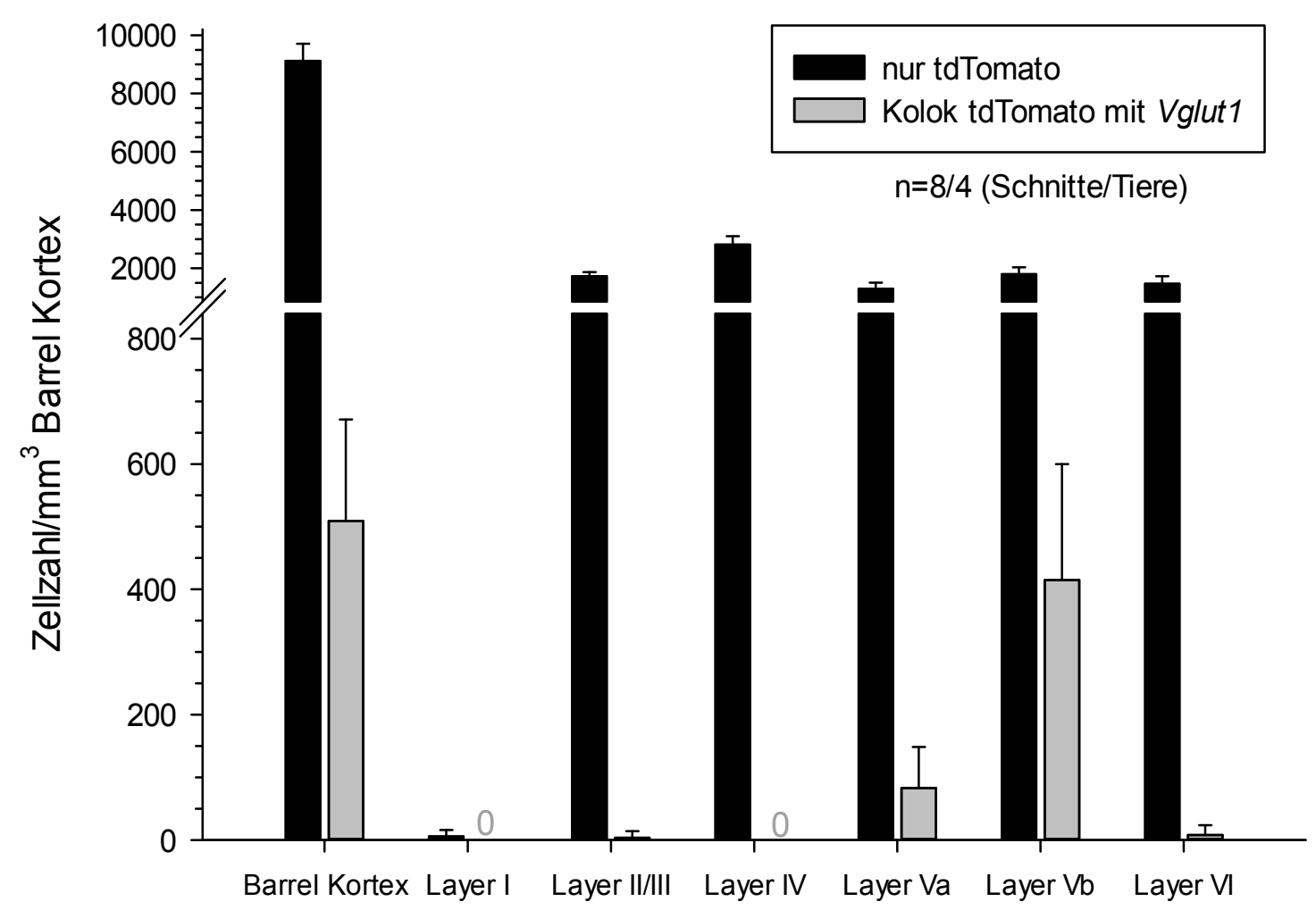

\section{Abbildung 3.7: Kolokalisation zwischen tdTomato-Zellen und der Vglut1-Sonde}

Die Balkendiagramme präsentieren für die einzelnen Schichten auf $1 \mathrm{~mm}^{3}$ Barrel-Kortex die Anzahl der Kolokalisationen zwischen den Zellen der PVcre/tdTomato-Maus und der Vglut1-Sonde (grau) sowie die tdTomato-Zellen ohne Markierung (schwarz).

Eine quantitative Auswertung der untersuchten acht Hirnschnitten soll bei der Bestimmung der schichtspezifischen Verteilung an Kolokalisation zwischen dem nativen tdTomato-Signal und der Vglut1-Sonde helfen (Abb. 3.7). Innerhalb des ausgezählten $1000 \mu \mathrm{m}$ breiten Bereiches des Barrel-Kortex sind von allen 9628,7 \pm 648,5 tdTomato-Zellen lediglich 508,9 \pm 162,1 Zellen pro $\mathrm{mm}^{3}$ Barrel-Kortex mit der Vglut1-Sonde kolokalisiert (Tab. 6.6). Prozentual gesehen entspricht das 5,3 \pm 1,6 \% aller tdTomato-Zellen (Abb. 3.8; Tab. 6.7). Auffällig daran ist, dass die kolokalisierten Zellen fast ausschließlich auf die Schichten Va und Vb begrenzt sind. Dabei findet man den signifikant größten Anteil an Kolokalisation in Schicht Vb mit 414,7 \pm 185,3 Zellen; gefolgt von Schicht Va mit 82,6 \pm 65,8 Zellen (Tab. 6.6., Tab. 6.14). Die Schicht Vb wies es in allen Hirnschnitten kolokalisierte Zellen auf. In Schicht Va wurde lediglich in einem von acht Hirnschnitten keine kolokalisierten Zellen gefunden. 
Betrachtet man den Anteil der mit der Vglut1-Sonde kolokalisierten tdTomato-Zellen gegenüber allen tdTomato-positiven Zellen in den einzelnen Schichten des Barrel-Kortexes, so weisen in Schicht Va 6,1 \pm 4,7 \% und in Schicht Vb 18,2 \pm 6,6 \% der tdTomato-Zellen eine Expression von Vglut1 auf (Abb. 3.8, Tab. 6.7). In den angrenzenden Schichten sind jeweils nur in einem Schnitt 0,2 \pm 0,6 kolokalisierte Zellen in Schicht II/III und in zwei Schnitten 0,6 \pm 1,2 kolokalisierte Zellen in Schicht VI zu finden (Tab. 6.7). Dementsprechend fällt die prozentuale Verteilung in Schicht II/III mit 0,2 \pm 0,6\% und in Schicht VI mit 0,8 $\pm 1,2 \%$ deutlich geringer aus und stellt wahrscheinlich ein methodisches Rauschen dar (Abb. 3.8).

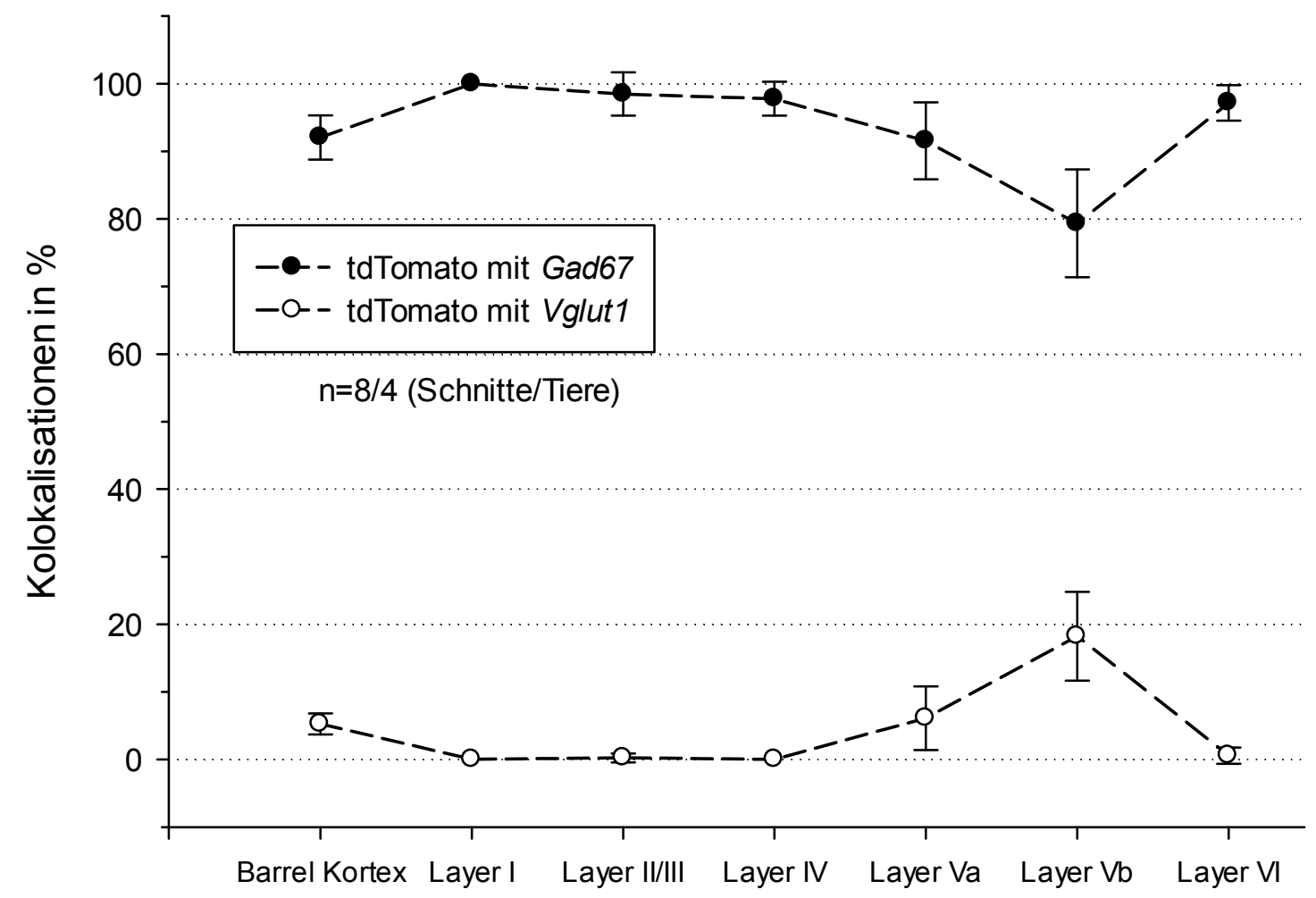

\section{Abbildung 3.8: Kolokalisation zwischen tdTomato mit der Gad1- und Vglut1-Sonde}

Der Graph zeigt den prozentualen Anteil der tdTomato-Zellen, die mit der Gad1- und der Vglut1-Sonde kolokalisiert sind. Alle Angaben sind auf $1 \mathrm{~mm}^{3}$ großen Bereich des Barrel-Kortex und den einzelnen Schichten bezogen.

In der schichtspezifischen Gegenüberstellung der beiden Sonden wird der komplementäre Verlauf der Kolokalisation verdeutlicht (Abb. 3.8). Der Graph der Vglut1-Sonde spiegelt sich in etwa in dem der Gad1-Sonde wider, was insbesondere für die Schichten Va und Vb auffällig ist. Der Anteil der detektierten $18 \%$ an Vglut1-positiven tdTomato-Zellen in der Schicht Vb sowie 
der ca. $7 \%$ in der Schicht Va entsprechen annähernd der $7 \%$ bzw. $18 \%$ der tdTomato-Zellen, die keine Gad1-Sonde aufweisen (Abb. 3.8). Dies lässt die Vermutung zu, dass die nicht kolokalisierten Gad1-markierten tdTomato-Zellen Vglut1-positiv sind.

Zusammenfassend lässt sich bestätigen, dass der größte Anteil der tdTomato-Zellen durch die Gad1-Sonde als inhibitorische Zellen klassifiziert werden können. Nur ein geringer Anteil besitzt keine Gad1-Markierung, überwiegend in den Schichten Va und Vb. Umgekehrt kann die Gegenprüfung mit Vglut1 bestätigen, dass die meisten tdTomato-Zellen nicht diesen exzitatorischen Marker exprimieren. Ebenfalls zeigen die Schichten Va und Vb eine signifikant erhöhte Kolokalisation der tdTomato-Zellen mit der Vglut1-Sonde. Daraus lässt sich ableiten, dass in der PVcre-Maus bis zu $18 \%$ der tdTomato-Zellen in den Schichten Va und Vb exzitatorische Pyramidenzellen darstellen und demzufolge keine GABAergen Interneurone (Abb. 3.8). Um diesen Ansatz weiter zu verfeinern, wurde im nächsten Schritt molekulare Marker für andere GABAerge Interneurone eingesetzt.

\subsection{Kolokalisation von tdTomato-positiven Zellen mit inhibitorischen Markern}

GABAerge Interneurone lassen sich durch molekulare Marker in drei distinkte Subpopulationen einteilen. Dazu zählen die Parvalbumin-, die Somatostatin- und die Serotonin-3a Rezeptorexprimierenden Neurone. Zu Letzteren gehören die VIP-exprimierenden Interneurone. Die Verteilung der distinkten Interneuronpopulationen ist über den sechs Schichten des somatosensorischen Kortex unterschiedlich ausgeprägt.

In dieser Arbeit soll untersucht werden, ob die tdTomato-positiven Zellen der PVcre-Maus neben Parvalbumin (Abs. 3.1) ebenfalls SOM bzw. VIP im Barrel-Kortex exprimieren. Durch die Verwendung spezifischenr Vip- und Sst-Sonden in der FISH wurden die tdTomato-Zellen auf eine Kolokalisation mit dem Sondensignal hin untersucht. Die Auswertung erfolgte für jede Sonde anhand von 2 Hirnschnitten von jeweils 4 verschiedenen Tieren (acht Schnitte pro Sonde). Mit Hilfe der Zellkerndarstellung (DAPI Färbung) konnten die neokortikalen Schichten abgegrenzt werden (siehe Material und Methoden).

In den Aufnahmen frontaler Hirnschnitte, die mit der Vip-Sonde inkubiert wurden, sind im Bereich des Barrel-Kortex der PVcre/tdTomato-Maus unterschiedlich angefärbte Neuronenpopulationen zu erkennen (Abb. 3.9). So lässt sich bereits in der Übersichtsaufnahme eine entgegengesetzte schichtspezifische Verteilung der nativen tdTomato-Zellen und der positiven VIP-Zellen vermuten (Abb. 3.9A). Grün fluoreszierende VIP-Zellen verteilen sich größtenteils in den oberen Schichten des Kortex. Hingegen liegt der hohe Präsenz der roten 
tdTomato-Zellen durch ihre Darstellung der Tönnchen in Schicht IV sowie in Schicht V. Dieser Unterschied wird in der 25fachen Vergrößerung eindeutiger (Abb. 3.9B). Der größte Anteil der VIP-positiven Zellen zeigt sich vermehrt in der Schicht II/III. Zusätzlich verdeutlicht die detailliertere Aufnahme aus Schicht II/III und IV, dass keine Kolokalisation zwischen den Zellgruppen vorliegt (Abb. 3.9C-C'). Das native tdTomato-Signal (Pfeilspitzen) und das grüne VIP-Signal der Sonde (Pfeile) liegen nicht übereinander. Zudem ist ersichtlich, dass das Vorkommen der VIP-Zellen in ihrer Gesamtzahl geringer ausfällt als das der überwiegend tdTomato-positiven PV-Zellen (Abb. 3.9B).
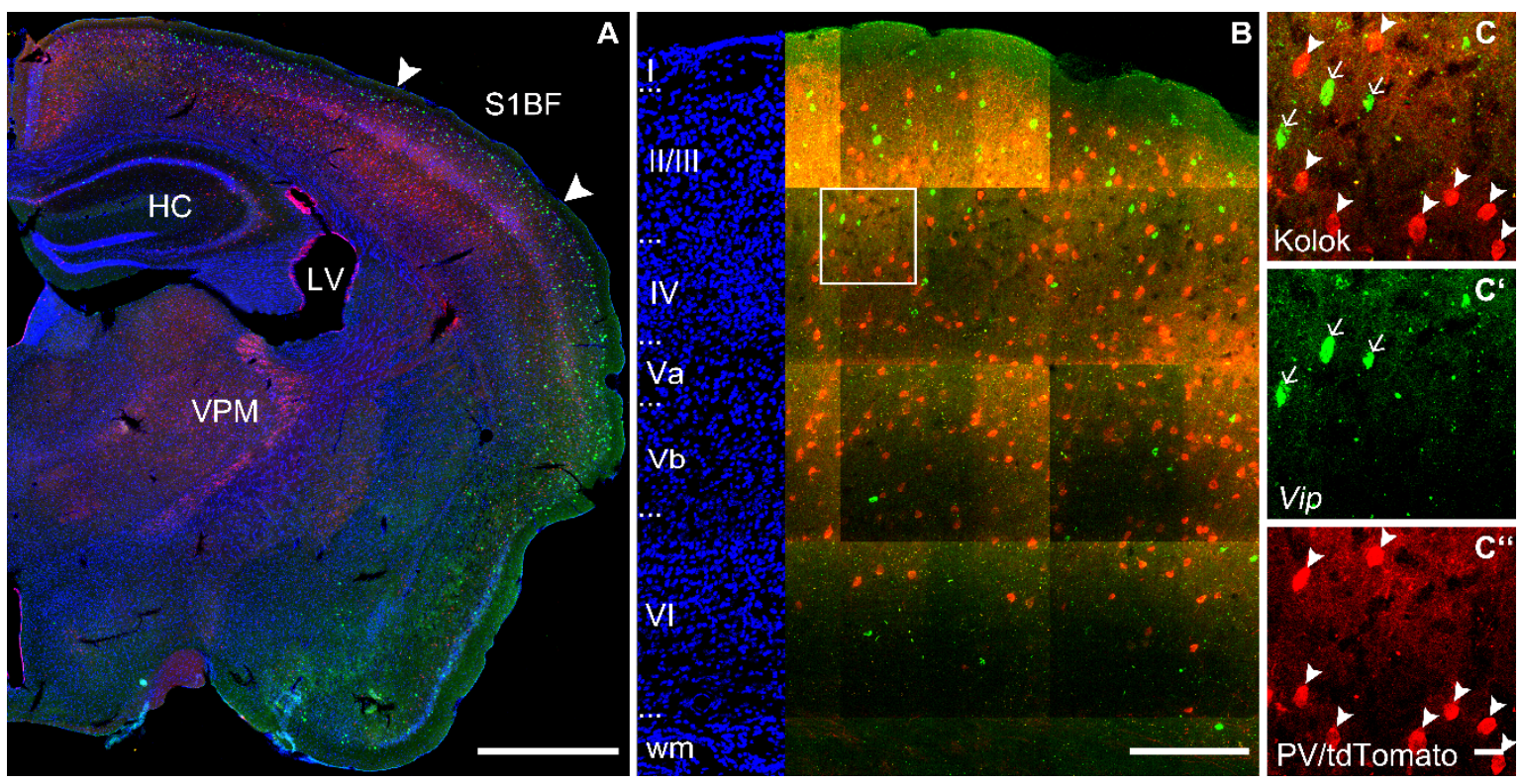

Abbildung 3.9: FISH zur Darstellung VIP-positiver Zellen im Bereich des Barrel-Kortex der PVcre/tdTomato-Maus

Fluoreszenz-in-situ-Hybridisierung mittels $V i p$-Sonde an frontalen Hirnschnitten einer PVcre/tdTomato-Maus, bei der in Rot das transgene tdTomato-Signal, in Grün die VIP-positiven Zellen, in Blau die Zellkerne (DAPI) und in Gelb die Kolokalisation zu erkennen sind. In $\mathbf{A}$ ist eine Einzelebene in der 10fachen Vergrößerung der linken Hemisphäre im Bereich des Barrel-Kortex (Pfeilspitzen; S1BF) dargestellt; Maßstab $1000 \mu \mathrm{m}$. Des Weiteren sind die Strukturen Hippocampus (HC), lateraler Ventrikel (LV) und der Thalamuskern Nucleus ventralis posteromedialis (VPM), zu sehen. (B) In der maximum intensity projection des Barrel-Kortex in der 25fachen Vergrößerung sind die neokortikalen Schichten durch römische Zahlen angegeben; Maßstab $200 \mu \mathrm{m}$. Der markierte Bereich wird in C-C" vergrößert wiedergegeben; Maßstab $20 \mu \mathrm{m}$. $\mathbf{C}$ zeigt das separate Vorkommen des tdTomato und des Vip-Sonde Signals, $\mathbf{C}^{\prime}$ zeigt die mit der Vip-Sonde markierten Zellen (Pfeile) und $\mathbf{C}^{\prime \prime}$ das native tdTomato-Signal. Die Pfeilspitzen markieren die tdTomato-positiven Zellen, wohingegen die Pfeile auf ausschließlich mit der Sonde markierten Zellen verweisen (C-C').

Die quantitative und schichtspezifische Auswertung der acht untersuchten Hirnschnitte bekräftigt die visuelle Nicht-Überlappung der VIP-Zellen mit den nativen tdTomato-Zellen. Innerhalb eines $1 \mathrm{~mm}^{3}$ großen Bereichs des Barrel-Kortex der PV/tdTomato-Maus konnten 
im Mittel 2927,7 \pm 580,0 VIP-Zellen detektiert werden, von denen 2906,9 \pm 587,4 Zellen ausschließlich eine grüne Fluoreszenz aufweisen und lediglich 20,8 \pm 27,0 Zellen mit dem tdTomato-Signal kolokalisiert sind (Abb. 3.10, Tab. 6.8, Tab. 6.9). Betrachtet man die Gesamtzahl der tdTomato-positiven Zellen von 10264,6 \pm 834,5 in diesen Schnitten, so besitzen lediglich 0,2 \pm 0,2 \% der tdTomato-Zellen eine VIP-Markierung (Abb. 3.13, Tab. 6.8, Tab. 6.10). Die sehr wenigen VIP-positiven tdTomato-Zellen sind ausschließlich in der Schicht II/III mit 20,8 \pm 27,0 Zellen zu finden (Tab. 6.9). Zudem kommen in nicht allen acht Hirnschnitten Kolokalisationen vor. In Schicht II/III konnten lediglich in $50 \%$ der ausgewerteten Schnitte, d. h. in vier von acht Schnitten kolokalisierte Zellen beobachten werden. Die VIP-positiven Zellen sind überwiegend in der Schicht II/III zu finden (1770,2 \pm 241,2 Zellen), was einem prozentualen Anteil von 60,5 \pm 8,2 \% entspricht (Tab. 6.8). Generell konnten im Neokortex in allen Schichten VIP-exprimierende Zellen nachgewiesen werden; Schicht IV 520,3 \pm 207,0 (17,8 \pm 7,1 \%); Schicht Va 86,9 \pm 60,6 (3,0 \pm 2,1 \%); Schicht Vb 222,4 \pm 135,4 (7,6 \pm 4,6 \%); Schicht VI 287,1 \pm 103,7 (9,8 \pm 3,5 \%) und Schicht I 47,7 \pm 34,6 $(1,6 \pm 1,2 \%)$. Anhand der durchgeführten Versuche und der Analyse kann geschlussfolgert werden, dass die tdTomato-positiven Zellen der PVcre-Maus weitestgehend kein VIP exprimieren und es sich bei den PV- und VIP-exprimierenden Interneuronen im Barrel-Kortex der Maus um distinkte Subpopulationen handelt. 


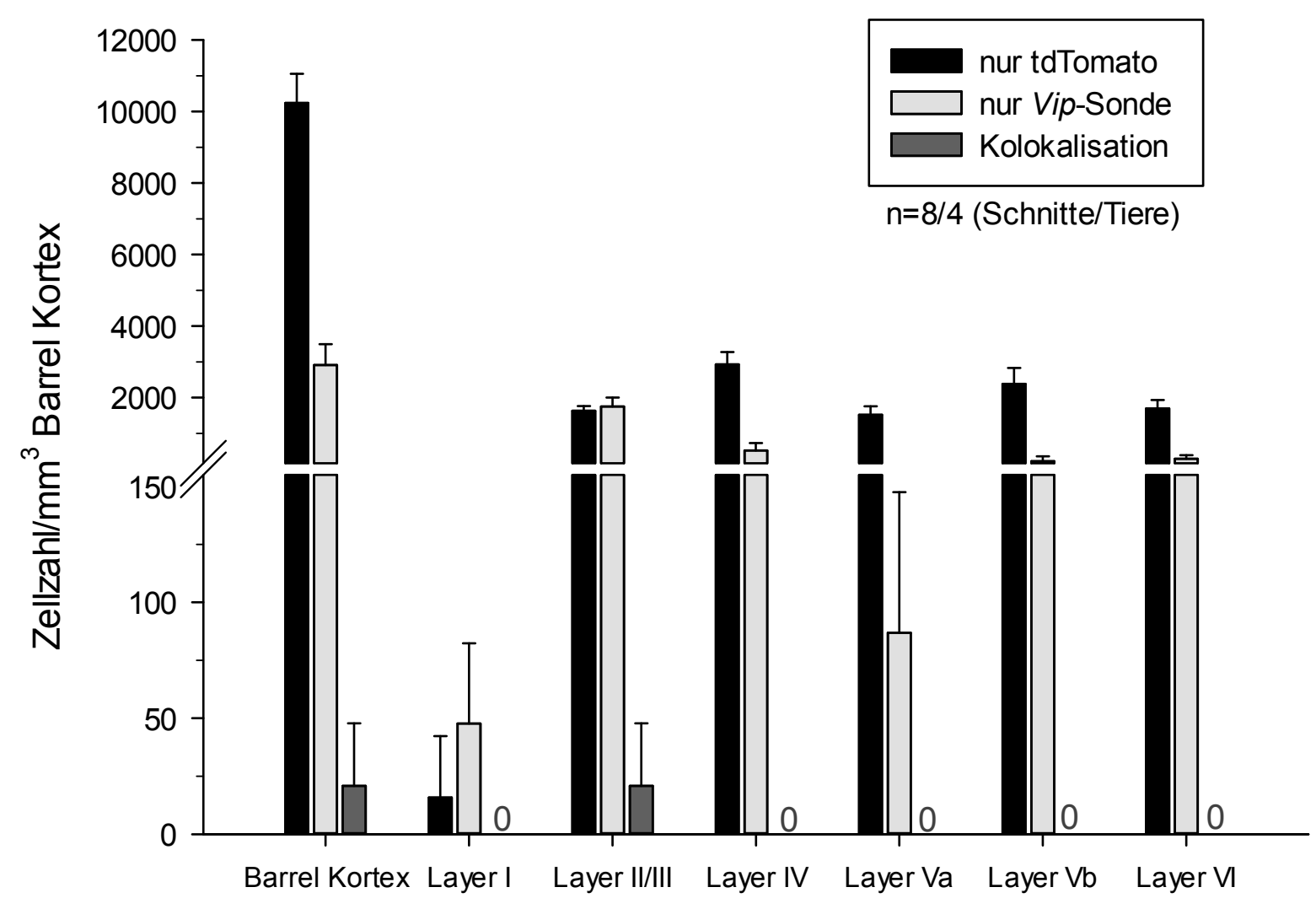

Abbildung 3.10: Kolokalisationen zwischen tdTomato und der Vip-Sonde

Die Balkendiagramme präsentieren innerhalb der einzelnen Schichten auf $1 \mathrm{~mm}^{3}$ Barrel-Kortex die Anzahl der Kolokalisationen zwischen den Zellen der PVcre/tdTomato-Maus und der Vip-Sonde (dunkelgrau), die tdTomato-Zellen ohne Markierung (schwarz) sowie die Zellen, die nur durch die VipSonde angefärbt wurden (hellgrau).

Analog der zuvor beschriebenen schichtspezifischen Kolokalisationsuntersuchung der VipSonde an der PVcre/tdTomato-Maus, wurde die Sst-Sonde eingesetzt. In der Übersichtsaufnahme eines frontalen Hirnschnittes zeigt sich in Grün das vermehrte Vorkommen der SOM-positiven Zellen, die die zweitgrößte inhibitorische Zellpopulation im Neokortex ausmacht (Abb. 3.11). Verglichen mit den nativen rot fluoreszierenden tdTomatoZellen, die durch die Abbildung der Tönnchen in Schicht IV markant herausstechen, lässt sich ein ubiquitäres Verteilungsmuster der grünen SOM-exprimierenden Zellen erkennen. Die 25 fache Vergrößerung aus dem Bereich des Barrel-Kortex veranschaulicht das überwiegend getrennte Vorkommen der SOM-positiven Zellen und der tdTomato-Zellen innerhalb der kortikalen Schichten (Abb 3.11B). Dieser Befund soll durch einen vergrößerten Ausschnitt aus Schicht IV hervorgehoben werden (Abb. 3.11C-C'). Die separate Darstellung beider Signale zeigt deutlich, dass die Zellsomata nicht aufeinanderliegen. Die jeweilige exakte Lage der 
tdTomato-positiven Zellen ist durch Pfeilköpfe und der SOM-positiven Zellen durch Pfeile markiert (Abb. 3.11C'-C'). In der qualitativen Darstellung wird die schichtspezifische Verteilung der SOM-Zellen und ihrer überwiegenden Lage in den Schichten Va und Vb sowie der Schicht IV deutlich (Abb. 3.11B).
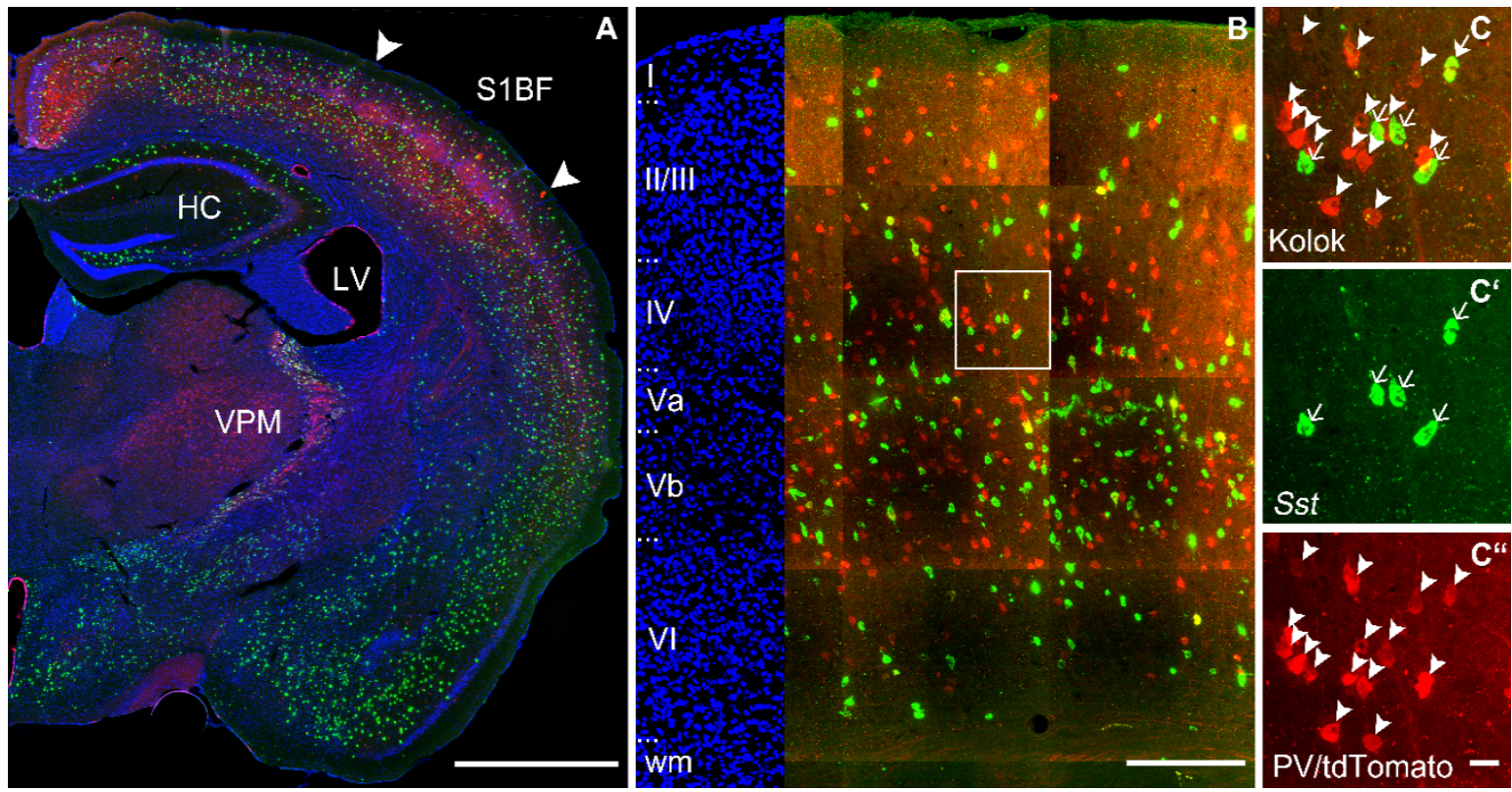

Abbildung 3.11: FISH zur Darstellung SOM-positiver Zellen im Bereich des Barrel-Kortex der PVcre/tdTomato-Maus

Fluoreszenz-in-situ-Hybridisierung mittels Sst-Sonde an frontalen Hirnschnitten einer PVcre/tdTomato-Maus, bei der in Rot das transgene tdTomato-Signal, in Grün die SOM-positiven Zellen, in Blau die Zellkerne (DAPI) und in Gelb die Kolokalisation zu erkennen sind. In $\mathbf{A}$ ist eine Einzelebene in der 10fachen Vergrößerung der linken Hemisphäre im Bereich des Barrel-Kortex (Pfeilspitzen; S1BF) dargestellt; Maßstab $1000 \mu \mathrm{m}$. Des Weiteren sind die Strukturen Hippocampus (HC), lateraler Ventrikel (LV) und der Thalamuskern Nucleus ventralis posteromedialis (VPM), zu sehen. (B) In der maximum intensity projection des Barrel-Kortex in der 25fachen Vergrößerung sind die neokortikalen Schichten durch römische Zahlen angegeben; Maßstab $200 \mu \mathrm{m}$. Der markierte Bereich wird in C-C" vergrößert wiedergegeben; Maßstab $20 \mu \mathrm{m}$. C zeigt das überwiegende separate Vorkommen des

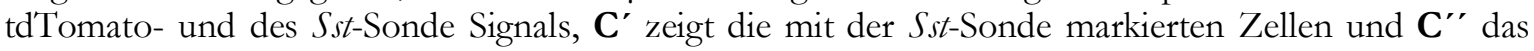
native tdTomato-Signal. Die Pfeilspitzen markieren die tdTomato-positiven Zellen sowie die mit der $S_{s t-}$ Sonde kolokalisierten tdTomato-positiven Zellen, wohingegen die Pfeile auf ausschließlich mit der Sonde markierten Zellen verweisen $\left(\mathbf{C}-\mathbf{C}^{\prime \prime}\right)$.

In der schichtenspezifischen Auswertung aller acht untersuchten Hirnschnitte wird deutlich, dass insbesondere in den infragranulären Schichten SOM-positive tdTomato-Zellen vorkommen. Die Auszählung ergibt 6730,8 \pm 741,6 SOM-exprimierende-Zellen auf einen 1 $\mathrm{mm}^{3}$ Bereich des Barrel-Kortex der PVcre/tdTomato-Maus, von denen 559,5 \pm 245,2 Zellen mit dem nativen tdTomato-Signal kolokalisiert sind (Abb. 3.12, Tab. 6.8). Das entspricht 5,3 \pm

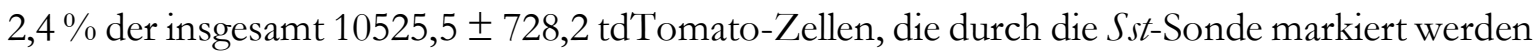


(Abb. 3.13, Tab. 6.8, Tab. 6.10). In der schichtenbezogenen Verteilung konnte das größte Vorkommen an SOM-positiven Zellen in der Schicht Vb mit 32,9 \pm 6,0 \% gefolgt von der Schicht Vb mit 21,3 \pm 9,7 \% registriert werden. Das entspricht in Schicht Vb 2214,2 \pm 402,8 und in Schicht VI 1431,0 \pm 651,8 SOM-positiven Zellen, von denen 142,2 \pm 130,6 bzw. 166,7 \pm 97,2 Zellen eine Kolokalisation mit dem tdTomato-Signal zeigen (Tab. 6.8, Tab. 6.9). Dahinter folgt die Schicht Va mit insgesamt 1033,6 \pm 207,9 und die Schicht IV mit 986,5 \pm 148,9 SOM-positiven Zellen. Hier lassen sich annähernd ähnliche Werte von 70,9 \pm 14,9 bzw. 115,6 \pm 41,8 kolokalisierten Zellen finden. Zuletzt folgt die Schicht II/III mit insgesamt 759,2 \pm 117,5 SOM-positiven Zellen und 57,3 \pm 47,4 Kolokalisationen. In Schicht II/III wurde nur in sechs von acht Hirnschnitten Kolokalisationen gezählt. In der Schicht I konnten nur in 50 $\%$, d. h. in vier von acht Schnitte SOM-positiven Zellen $(32,6 \pm 52,7)$ registriert werden, von denen keine mit tdTomato kolokalisiert sind.

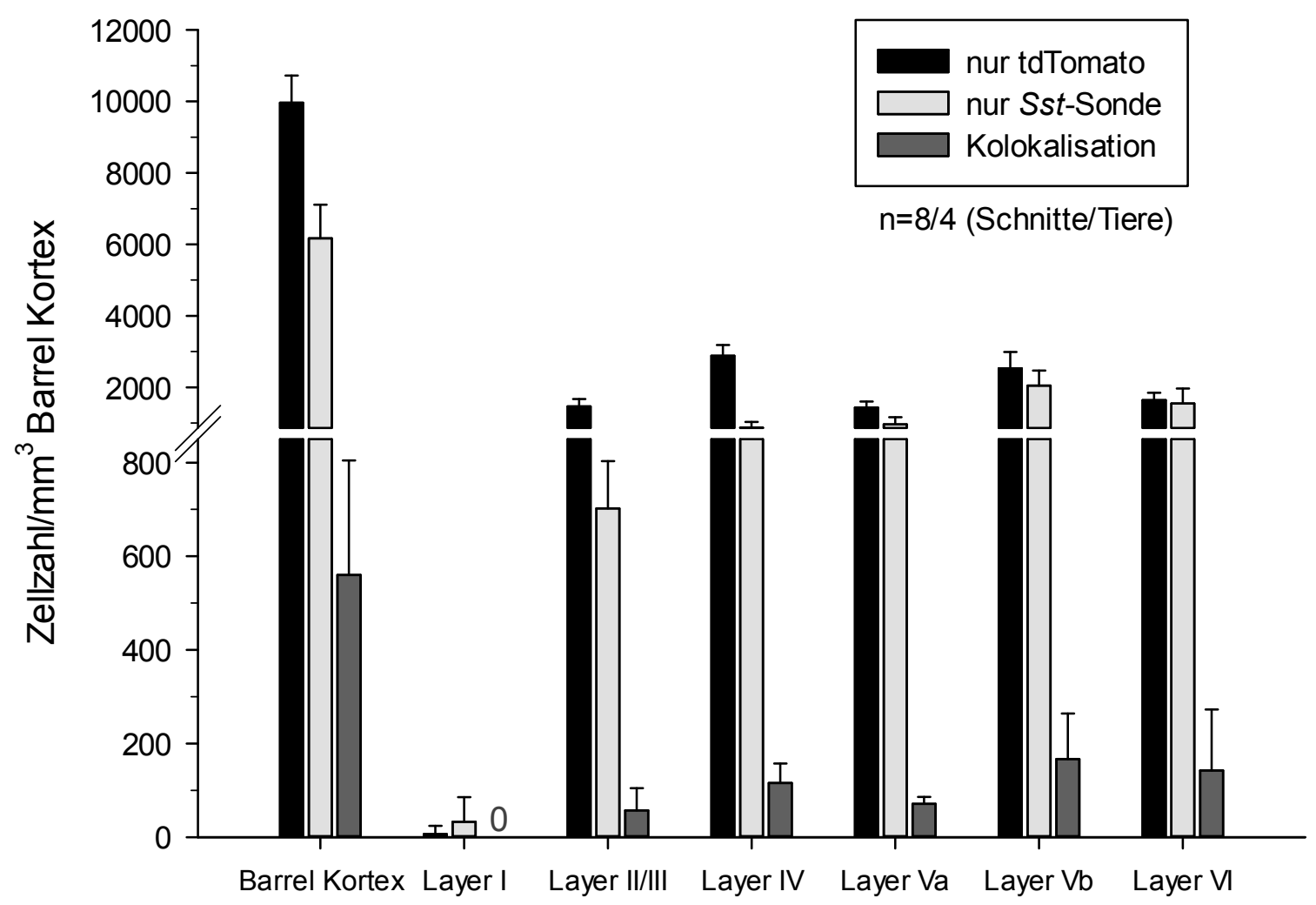

\section{Abbildung 3.12: Kolokalisationen zwischen tdTomato und der Sst-Sonde}

Die Balkendiagramme präsentieren innerhalb der einzelnen Schichten auf $1 \mathrm{~mm}^{3}$ Barrel-Kortex die Anzahl der Kolokalisationen zwischen den Zellen der PVcre/tdTomato-Maus und der Sst-Sonde

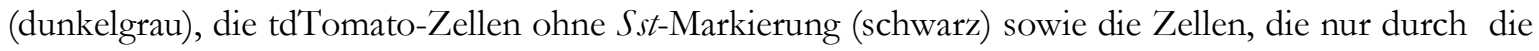
Sst-Sonde markiert wurden (grau). 
Die prozentuale Darstellung der Kolokalisationsquote der tdTomato-positiven Zellen mit den drei untersuchten inhibitorischen Markern (Parvalbumin-Antikörper, Sst- und Vip-Sonde) in der PVcre-Maus zeigt, dass der Großteil von 87,6 \pm 6,7 \% der tdTomato-Zellen im Barrel-Kortex immunpositiv für Parvalbumin ist (Abb. 3.13, Tab. 6.3). Ausgenommen der Schicht I, in der alle tdTomato-Zellen Parvalbumin exprimieren, liegen die Werte der anderen Schichten in einem ähnlichen Bereich von über 87,0 \%.

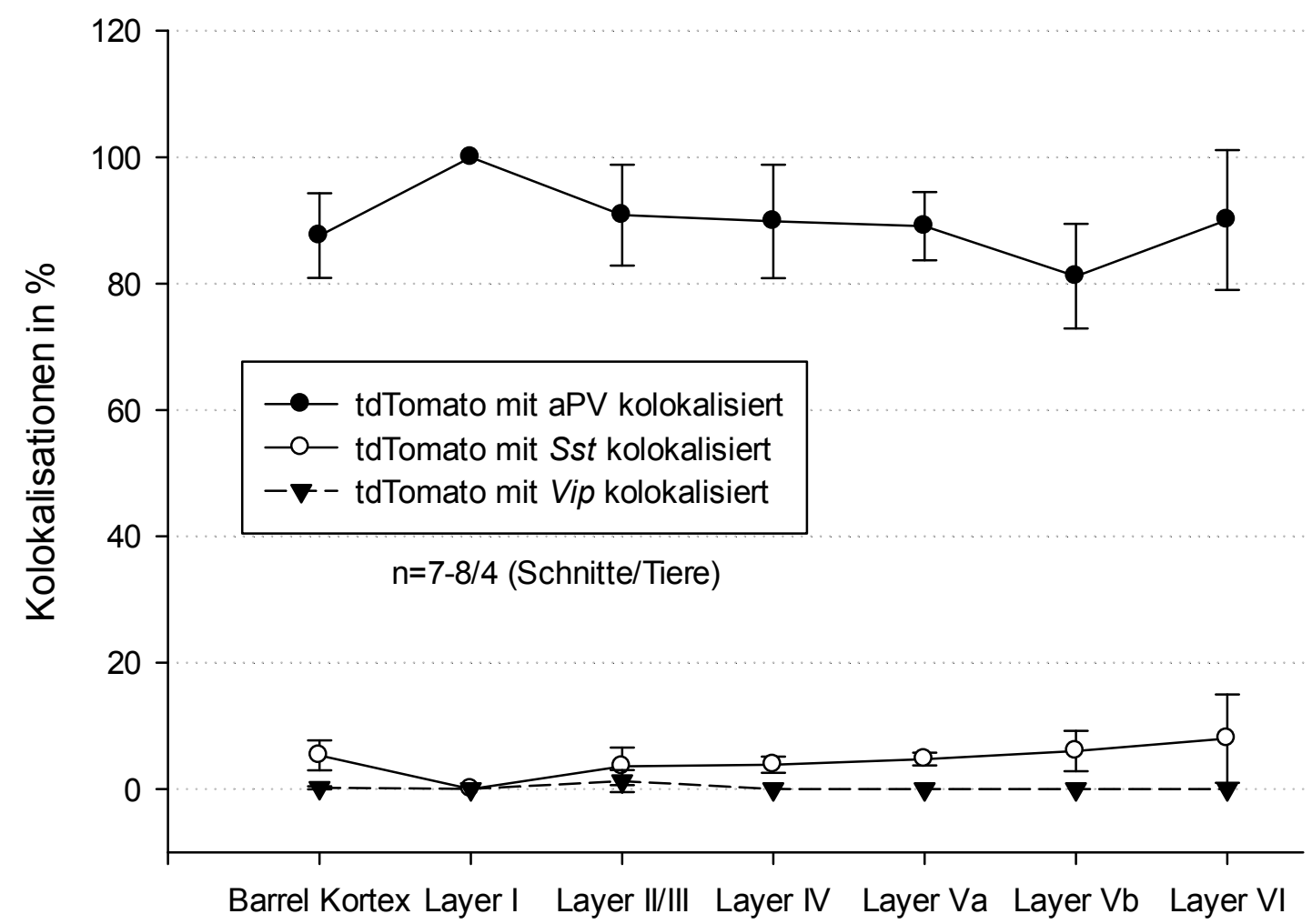

\section{Abbildung 3.13: Vergleich der prozentualen schichtenspezifischen Kolokalisation zwischen den tdTomato-Zellen und dem PV-Antikörper, der Sst-sowie der Vip-Sonde}

Der Graph zeigt den prozentualen Anteil der tdTomato-Zellen, die mit dem PV-Antikörper, der $S_{s t-}$ und Vip-Sonde kolokalisiert sind. Alle Angaben sind auf $1 \mathrm{~mm}^{3}$ großen Bereich des Barrel-Kortex und den einzelnen Schichten bezogen.

Umgekehrt zur PV-Markierung weisen die tdTomato-Zellen nur eine sehr geringe Überlappung mit der Vip-Sonde auf von 0,2 \pm 0,2 \% im gesamten Barrel-Kortex. Sie beträgt in der Schicht II/III 1,3 \pm 1,7 \% (Abb. 3.13, Tab. 6.10). Im Gegensatz zur Vip-Sonde scheinen mehr tdTomato-Zellen Somatostatin zu exprimieren, insbesondere in den infragranulären Schichten (Abb. 3.13). Von den im gesamten Barrel-Kortex 5,3 $\pm 2,4 \%$ an SOM-positiven tdTomatoZellen sind die meisten in der Schicht VI lokalisiert $(8,0 \pm 7,0 \%$ ). Schichtenbezogen nimmt der 
Prozentsatz der SOM-positiven tdTomato-Zellen von der Schicht II/III mit 3,6 \pm 3,0 \% bis zur Schicht VI stetig zu (Abb. 3.13, Tab. 3.6.10). Die sehr hohe Kolokalisationsquote in der Schicht VI ist in der Tatsache begründet, dass in einer von vier Mäusen es in einem ausgezählten Schnitten bis zu $18 \%$ sowie in dem Weiteren bis $36 \%$ der tdTomato-Zellen mit der $S_{s t-S o n d e}$ markiert sind, und in den restlichen sechs Hirnschnitten lediglich 1-12\%.

Schlussfolgernd kann nach der gesamten quantitativen Auswertung festgehalten werden, dass es sich bei den tdTomato-Zellen der PVcre-Maus vorrangig um inhibitorische Parvalbuminexprimierende Interneurone handelt. Wobei insbesondere in den infragranulären Schichten der prozentuale Anteil an exzitatorischen Neuronen (Schicht V) und Somatostatin- exprimierenden Interneuronen (Schicht V-VI) hoch ausfällt und somit die Spezifität der PVcre-Maus stark einschränkt.

\subsection{PV-Neurone tragen perineuronale Netze}

Bisherige Studien haben herausgefunden, dass sich bevorzugt um die Somata schnell-feuernde Neurone perineuronale Netze (PNN) befinden können. Diese PNN sind spezialisierte Komplexe von extrazellulären Matrixmolekülen. Chondroitinsulfat-Proteoglykane (CSPGs), das auch zu der Familie der Lektikane gehört, ist ein wesentlicher Bestandteil von PNN. Zur Visualisierung bedient man sich des Lektins Wisteria floribunda Agglutinin (WFA), dass an die terminalen N-Acetylgalactosaminreste von CSPGs bindet. In dieser Studie sollen mithilfe von

WFA die tdTomato-Zellen hinsichtlich des Vorkommens perineuronaler Netze schichtenspezifisch untersucht werden. Hierfür wurden analog zu den bisherigen Färbungen acht Schnitte von vier verschiedenen PVcre-Mäusen untersucht.

Die Übersichtsaufnahme der WFA-Färbung eines frontalen Hirnschnittes zeigt durch die grüne Fluoreszenz eine hohe Präsenz perineuronaler Netze im Nucleus reticularis (Rt), in der Fasciola cinereum (FC) sowie im Retrosplenialen Kortex (RSC) (Abb. 3.14A). Auch im Barrel-Kortex finden sich besonders viele PNN. Der Nucleus ventralis posteromedialis (VPM) und die Fasern des Tractus opticus stechen wiederum mit ihrer roten tdTomato-Fluoreszenz stark hervor. Die 25fache Vergrößerung zeigt eine weitgehend auf die Schicht IV und Vb konzentrierte WFAFärbung der perineuronalen Netze (Abb. 3.14B). Dabei ist auffällig, dass die PNN nahezu ausschließlich um die tdTomato-positiven Zellen vorkommen. Neben dem dominanten Vorkommen von kolokalisierten tdTomato-Zellen in den Schichten IV und Vb, lassen sich in den Schichten II/III, Va und VI einzelne kolokalisierte tdTomato-Zellen finden. In der höheren Auflösung des markierten Bereichs aus Schicht IV kann anhand der Signalseparierung die 
Umrandung der tdTomato-positiven Somata durch die perineuronalen Netze bestätigt werden (Abb. 3.14C). Die Pfeilköpfe zeigen die tdTomato-Zellen und die Pfeile die PNN, welche in der Überlagerung komplett Verschmelzen (Abb. 3.14C).
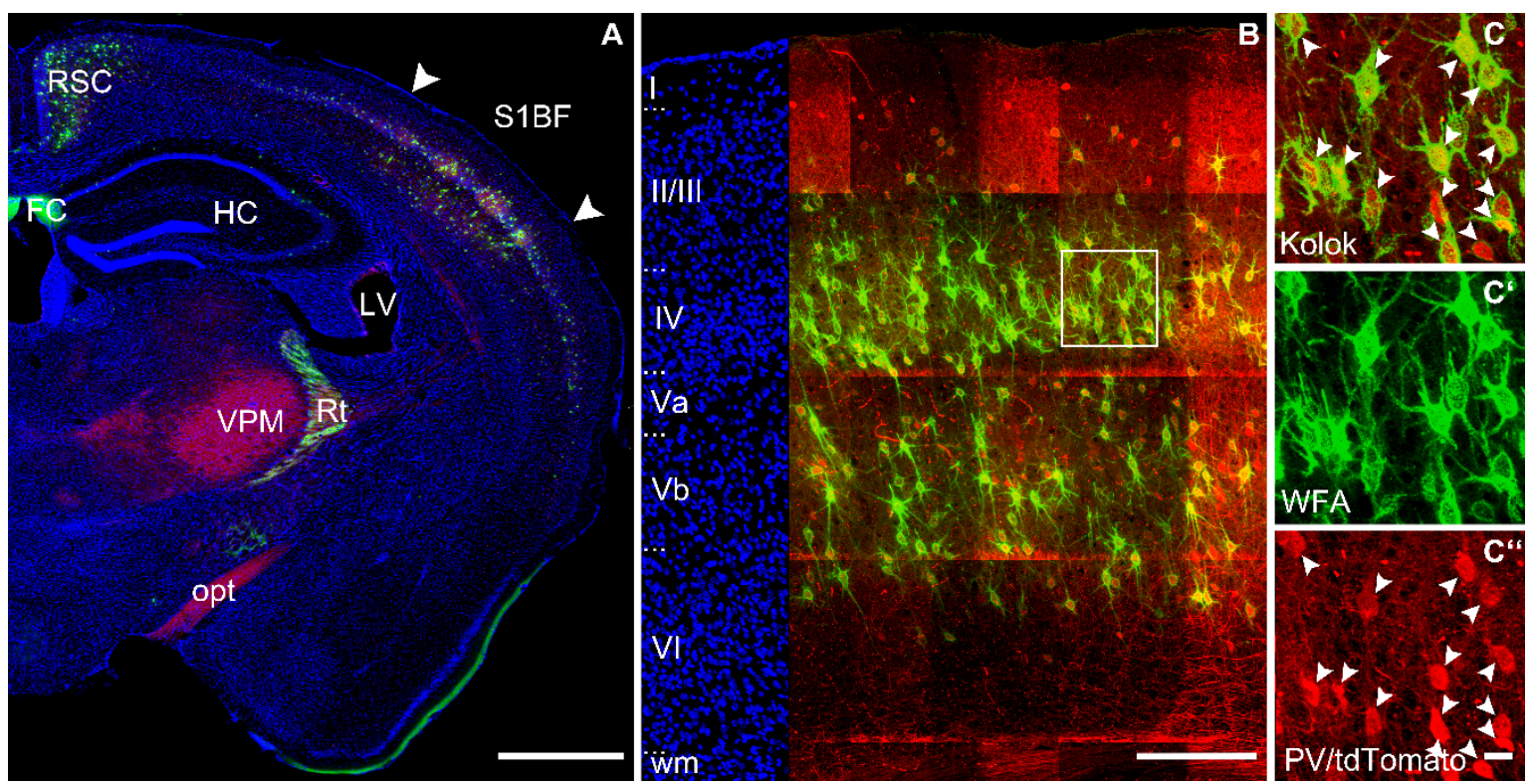

Abbildung 3.14: WFA-Färbung zur Darstellung perineuronaler Netze im Bereich des BarrelKortex der PVcre/tdTomato-Maus

WFA-Färbung an frontalen Hirnschnitten einer PVcre/tdTomato-Maus, bei der in Rot das transgene tdTomato-Signal, in Grün der WFA-positiven Zellen, in Blau die Zellkerne (DAPI) und in Gelb die Kolokalisation zu erkennen sind. In $\mathbf{A}$ ist eine Einzelebene in der 10fachen Vergrößerung der linken Hemisphäre im Bereich des Barrel-Kortex (Pfeilspitzen; S1BF) dargestellt; Maßstab $1000 \mu \mathrm{m}$. Des Weiteren sind die Strukturen Hippocampus (HC), lateraler Ventrikel (LV), Retrosplenialer Cortex (RSC), Tractus opticus (opt), Fasciola cinereum (FC) und die Thalamuskerne, Nucleus ventralis posteromedialis (VPM) und Nucleus reticularis (Rt), zu sehen. (B) In der maximum intensity projection des Barrel-Kortex in der 25fachen Vergrößerung sind die neokortikalen Schichten durch römische Zahlen angegeben; Maßstab $200 \mu \mathrm{m}$. Der markierte Bereich wird in C-C"' vergrößert wiedergegeben; Maßstab $20 \mu \mathrm{m}$. C zeigt die Umrandung der tdTomato-positiven Zellen durch das WFA-Signal in Gelb, $\mathbf{C}^{\prime}$ zeigt die WFAmarkierten Zellen und $\mathbf{C}^{\prime \prime}$ das native tdTomato-Signal. Die Pfeilspitzen markieren die mit der WFAFärbung umrandeten tdTomato-positiven Zellen (C-C').

In der quantitativen Auswertung der acht Hirnschnitte konnten 9748,5 \pm 513,2 tdTomatopositive Zellen pro $1 \mathrm{~mm}^{3}$ Barrel-Kortex detektiert werden. Von diesen tragen 6909,6 \pm 587,3 Zellen ein perineuronales Netz (Abb. 3.15, Tab. 6.11), was 70,9 \pm 5,6 \% der tdTomato-Zellen entspricht (Abb. 3.16, Tab. 6.13, Tab. 6.14). Insgesamt konnten 6964,3 \pm 575,1 WFAmarkierten Zellen in $1 \mathrm{~mm}^{3}$ nachgewiesen werden, wovon 99,2 \pm 0,9 \% der PNNs ausschließlich, um tdTomato-Zellen zu finden sind (Abb. 3.16, Tab. 6.11, Tab. 6.13).

Hinsichtlich der schichtspezifischen Verteilung der insgesamt 70,9 \pm 5,6 \% PNN-positiven tdTomato-Zellen kommen in der Schicht IV mit 36,7 \pm 2,0 \% (2538,0 \pm 136,1 Zellen) der 
Großteil dieser Zellen vor (Tab. 6.11, Tab. 6.12). Die zweitgrößte Population entfällt, wie in der qualitativen Darstellung vermutet, auf die Schicht Vb mit 1424,3 \pm 209,8 bzw. 20,6 \pm 3,0 \%. Dahinter folgen die Schichten Va mit 1066,6 \pm 179,6 Zellen $(15,4 \pm 2,6 \%)$ und die Schicht II/III mit 1059,4 \pm 95,8 Zellen (15,3 \pm 1,4 \%). In der Schicht VI kommen lediglich 808,8 \pm 224,9 Zellen $(11,7 \pm 3,3 \%)$ der PNN-positiven tdTomato-Zellen vor. In Schicht I sind keine PNNs zu beobachten (Tab. 6.11, Tab. 6.12).

Insgesamt konnten lediglich in fünf von acht Hirnschnitten nicht mit tdTomato-Zellen kolokalisierte PNN-positive Zellen (54,8 \pm 58,7 Zellen) beobachtet werden, die sich wie folgt verteilten: 3,5 \pm 9,9 Zellen in Schicht II/III in einem Schnitt; 15,5 \pm 25,5 Zellen in Schicht IV in drei Schnitten; 6,4 \pm 11,8 Zellen in Schicht Va in zwei Schnitten; 19,1 \pm 22,9 Zellen in Schicht $\mathrm{Vb}$ in vier Schnitten und 10,3 \pm 20,6 Zellen in zwei Schnitten (Abb. 3.15).

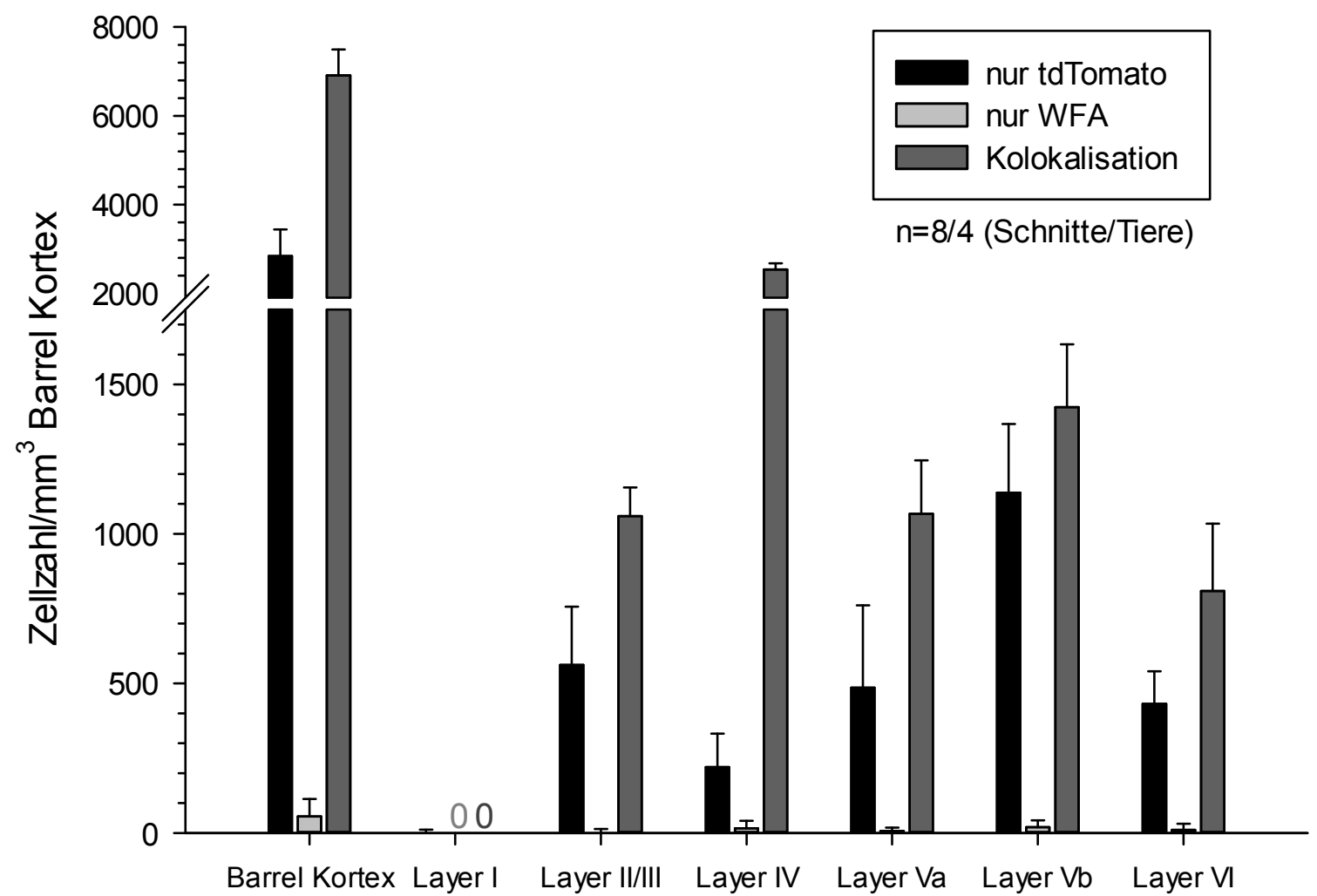

\section{Abbildung 3.15: Kolokalistaionen zwischen tdTomato und WFA}

Die Balkendiagramme präsentieren innerhalb der einzelnen Schichten auf $1 \mathrm{~mm}^{3}$ Barrel-Kortex die Anzahl der Kolokalisationen zwischen den Zellen der PVcre/tdTomato-Maus und WFA (dunkelgrau), die tdTomato-Zellen ohne Markierung (schwarz) sowie die Zellen, die nur durch WFA angefärbt wurden (grau). 
Die PNN liegen somit spezifisch an den tdTomato-Zellen und umschließen in typischer Weise den Zellkörper (Abb. 3.14 C). Insgesamt befinden sich um mehr als $70 \%$ aller tdTomato-Zellen im Barrel-Kortex perineuronale Netze (Abb. 3.16). Betrachtet man die einzelnen Schichten separat, so sind 92,1 \pm 3,9\% der tdTomato-Zellen in der Schicht IV mit WFA markiert. Damit weist die Schicht IV den signifikant höchsten Anteil an PNN tragender tdTomato-Zellen auf (Tab. 6.14). Dagegen weisen nur ca. 64-70 \% der tdTomato-Zellen in den Schichten II/III, Va und VI ein PNN auf. In der Schicht Vb sind es lediglich 55,7 \pm 6,0 \% der Zellen, d. h. nur der die Hälfte der tdTomato-Zellen tragen ein PNN. Dieses Ergebnis spiegelt das signifikante Vorkommen der perineuronalen Netze um die Parvalbumin-exprimierenden Zellen, insbesondere in der Schicht IV wider. Es bleibt die Frage offen, warum ausgerechnet die Schicht IV PV-exprimierenden Interneurone eine derart hohe Ansammlung von perineuronalen Netzen aufweisen, im Gegensatz zu den anderen kortikalen Schichten vorkommenden PV-Zellen.

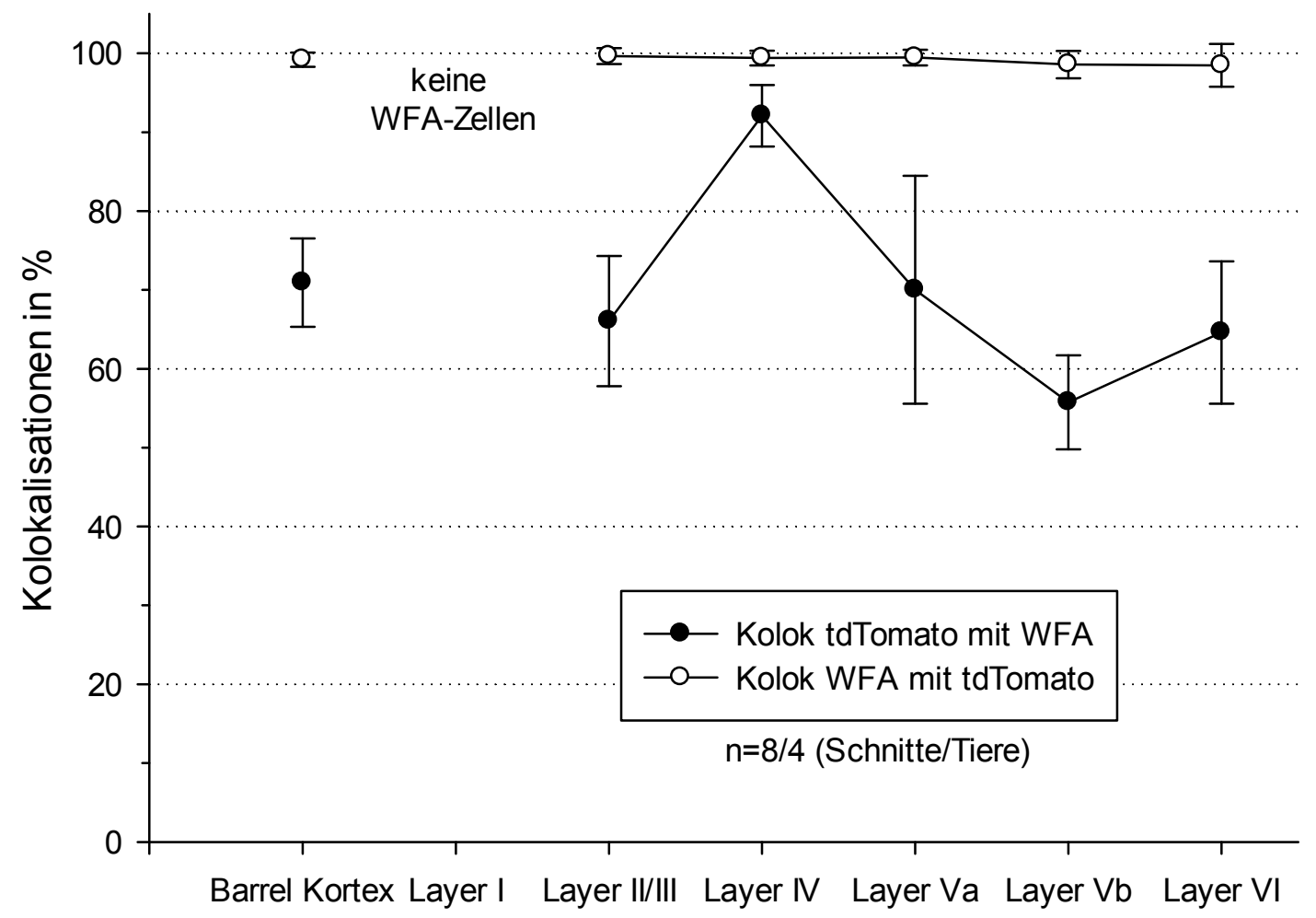

\section{Abbildung 3.16: Kolokalisation zwischen tdTomato und dem Antikörper WFA in \%}

Der Graph zeigt den prozentualen Anteil der tdTomato-Zellen, die mit dem WFA-Marker kolokalisiert sind. Alle Angaben sind auf $1 \mathrm{~mm}^{3}$ großen Bereich des Barrel-Kortex und den einzelnen Schichten bezogen. 


\section{Diskussion}

Die in dieser Arbeit gewonnenen Ergebnisse sollen dazu beitragen, das Grundwissen über die kortikalen Netzwerke und deren inhibitorische Interneurone auszubauen sowie das Verständnis der Funktionsvielfalt von GABAergen Interneuronen zu verbessern.

Der Fokus dieser Studie liegt in der Überprüfung der PVcre/tdTomato-Mauslinie auf Sensitivität sowie Spezifität der nativen tdTomato-Fluoreszenz im Hinblick auf ihre Identität als PV-exprimierende Interneurone. Weiterhin soll der Blick auf die schichtenspezifische Verteilung der tdTomato-Zellen im Bereich des Barrel-Kortex gerichtet werden und deren mögliche Kolokalisation mit den Neuropeptiden Somatostatin (SOM) und dem vasoaktiven intestinalen Peptid (VIP) ausgeschlossen werden. Zusätzlich wurden bei der Charakterisierung der PVcre/tdTomato-Maus die tdTomato-Zellen auf das Tragen perineuronaler Netze hin untersucht. Die Experimente basierten auf den Techniken der Histochemie, Immunhistochemie und Fluoreszenz-in-situ-Hybridisierung.

Aufgrund der Ergebnisse dieser Studie kann man Folgendes zusammenfassen:

Die rot fluoreszierenden tdTomato-Zellen im Barrel-Kortex der untersuchten PVcre/tdTomato-Mauslinie exprimieren überwiegend (87,6 \%) Parvalbumin, dabei befinden sich die meisten tdTomato-Zellen in den Schichten IV (28,7 \%) und Vb (24,7 \%). Zur Gruppe der Gad1-exprimierenden Neurone gehören überwiegend die tdTomato-Zellen (92\%) und sind demzufolge inhibitorische GABAerge Interneurone. In den Schichten Va und Vb konnten jedoch Vglut1-positive tdTomato-Zellen registriert werden, mit jeweils ca. $7 \%$ in Schicht $\mathrm{Va}$ und ca. $18 \%$ in Schicht Vb. Zwischen den tdTomato-Zellen und der Sst-Sonde besteht eine geringe Überlappung von 5,3\%, wohingegen die Vip-Sonde keine Kolokalisation zeigt (0,2 \%). Betrachtet man die tdTomato-positiven Neurone ist die Mehrheit von ihnen umgeben von perineuronalen Netzen (70\%). Verglichen dazu sind $99 \%$ aller perineuralen Netze (PNN) tragenden Zellen durch das tdTomato-Signal markiert. Dabei tragen in der Schicht IV 92,1\% der tdTomato-Zellen PNNs.

Dies bedeutet, dass die tdTomaten-Zellen der PVcre/tdTomato-Maus im Barrel-Kortex fast vollständig inhibitorische Eigenschaften aufweisen und zu den PV-exprimierenden GABAergen Neuronen hinzugezählt werden können. Die stärkste Präferenz der tdTomato-Zellen zeigt sich dabei in Schicht IV und Vb. Zudem können die perineurale Netze der tdTomato-positiven Neuronen als zusätzlichen Charakterisierungsmerkmal angesehen werden. 


\subsection{Methodische Gesichtspunkte}

\subsubsection{Immunhistochemie und Fluoreszenz-in-situ-Hybridisierung}

Die für die Studie verwendete Technik der Immunhistochemie basiert auf dem Nachweis von Makromolekülen mit Hilfe von Antikörpern, die an spezifische Epitope binden können. Diese Bindung erfolgt aufgrund ihrer Affinität und Spezifität für bestimmte Antigene, wie Proteine, Glykoproteine, Lipoproteine oder Polysaccharide (Mulisch und Welsch 2015). Der Nachweis der Bindung des Antikörpers an das entsprechende Antigen kann dabei durch eine direkte oder indirekte Immunmarkierung erfolgen. Die direkte Immunmarkierung wird auch Einschrittmethode genannt und koppelt den mit einem Marker versehenen Antikörper mit dem Epitop des Antigens (Mulisch und Welsch 2015). Diese Substanz kann unter anderem durch Fluoreszenz sichtbar gemacht werden. Die Vorteile dieser Methode zeigen sich in der einfachen und schnellen Durchführung, der fast vollständigen Ausschaltung unspezifischer Signale, dem Wegfall störender Hintergrundmarkierungen und der möglichen Mehrfachmarkierung (Mulisch und Welsch 2015). Im Gegensatz dazu benötigt man für die indirekte Immunmarkierung einen Sekundärantikörper, der durch die Bindung an den primären Antikörper die Aufgabe des Markers übernimmt. Dazu erfolgt die Herstellung des Sekundärantikörpers in einer anderen Tierspezies. Der große Vorteil der indirekten Methode liegt in der Signalverstärkung durch die zahlreichen Bindungsmöglichkeiten an den Erstantikörper (Mulisch und Welsch 2015). Durch die Verwendung von polyklonalen Antikörpern lässt sich das Signal-Rausch-Verhältnis durch die Möglichkeit an verschiedene Epitope der Zielzelle zu binden weiter verbessern. Hierin besteht allerdings auch die Fehleranfälligkeit dieser Methode, wenn es zu Kreuzreaktionen des sekundären Antikörpers kommt. Des Weiteren ist diese Methode auch besonders fehleranfällig für äußere Einflüsse, wie z. B. Temperatur, pH-Wert etc. In der durchgeführten Studie wurde die indirekte Immunmarkierung ausschließlich für das Protein Parvalbumin eingesetzt. Das genutzte Protokoll ist seit Jahren für diesen Antikörper etabliert (Prönneke et al. 2015). Der mRNA Nachweis der Neuropeptide Somatostatin und VIP, sowie der Enzyme Gad67 und Vglut1 erfolgte in einem zweiten Teil der Versuche mittels der Fluoreszenz-in-situHybridisierung.

Die In-situ-Hybridisierung ist eine Methode zur Lokalisation spezifischer DNA- oder RNASequenzen in einem fixierten Gewebsabschnitt (Mulisch und Welsch 2015). Dazu werden möglichst komplementäre Sonden, einsträngige Nucleinsäurefragmente, hergestellt, an die chemisch ein spezieller Marker gebunden ist. Durch ihn kann die spätere Detektion und 
Lokalisation der komplementären Zielsequenz stattfinden. Voraussetzung für einen erfolgreichen Einsatz der Sonden ist die Zugänglichkeit an die Zielmoleküle, wofür mehrere Arbeitsschritte notwendig sind. Zuerst werden die vorbehandelten Präparate, die komplementären Sonden, unter definierten Bedingungen denaturiert, sodass einwandfreie Hybridisierung stattfinden kann. Bei der Hybridisierung erfolgt die Paarung der komplementären Basen des Nukleinsäurestrangs, in diesem Fall die Paarung der zuvor hergestellten Sonde an RNA-Sequenzen mit dem jeweiligen Strang der zu untersuchenden mRNA der Zielzelle. Überschüssiges Material wird durch reichliche Waschvorgänge entfernt. An den Molekülen der Sonden wird Biotin und Avidin durch Bindung an Fluorophore indirekt markiert und kann so nachgewiesen werden. Schlussendlich kann die mikroskopische Darstellung der gebundenen Sequenzen unter dem jeweils spezifisch gefilterten fluoreszierenden Licht erfolgen. Die gesamten Arbeitsschritte der In-situ-Hybridisierung sind sehr empfindlich für äußere Einflüsse, insbesondere für RNAasen. Das FISH-Protokoll zum Nachweis der Sst- und Vip-mRNA sowie, der Gad1- (Gad67-) und Vglut1-mRNA ist analog zu bisher durchgeführten Versuchsreihen (Prönneke et al. 2015).

\subsubsection{Vergleich von Epifluoreszenz mit Apo'Tome und Konfokalmikroskopie}

In dieser Studie wurden alle dreidimensionalen Aufnahmen der zu analysierenden Hirnschnitte der PVcre/tdTomato-Maus mit einem Epifluoreszenzmikroskop mit strukturierter Beleuchtung, dem ApoTome, angefertigt. Die fluoreszenzmarkierten Präparate werden durch gefiltertes Licht einer Quecksilberdampflampe in einem spezifischen Wellenlängenbereich angeregt und das emittierte Licht über Filter auf eine Grauskalen-Kamera geleitet. Mit Hilfe der strukturierten Beleuchtung konnte das Streulicht in den 3D-Aufnahmen deutlich reduziert und dadurch ein höherer Kontrast und vor allem eine bessere Z-Auflösung erzielt werden, d.h. eine Reduktion von Streulicht aus anderen optischen Ebenen.

Im Allgemeinen kann die Fluoreszenzmikroskopie für immunhistochemische Färbungen und Fluoreszenz-in-situ-Hybridisierung verwendet werden. Bei Kolokalisationsstudien wie in dieser Arbeit, ist die Verwendung von im Spektrum weit entfernter Fluorophore und spezifischer Filter von entscheidender Bedeutung. Durch den Einsatz des ApoTomes in den 3D-Scans konnte eine nachweisbare Trennung der Signale erreicht werden (Prönneke et al. 2015). Zudem ist in einigen Experimenten beschrieben worden, dass es bei Proben mit einer zunehmenden Dicke von $>27 \mu \mathrm{m}$ zu Überstrahlungen aus anderen Ebenen und somit zu Ungenauigkeiten kommen kann (Scheuer 2015; Weigel et al. 2009). Um jedoch die Verlässlichkeit der 
Epifluoreszenzaufnahmen zu bestätigen, wurde von jedem Marker zusätzlich eine Aufnahme in 20facher Vergrößerung mit einem konfokalen Laser-Scanning-Mikroskop erstellt (Abb. 4.1). Das Präparat wurde mittels eines fokussierten Laserstrahls abgerastert und konnte die erzeugte Fluoreszenz mit optimaler XYZ-Auflösung aufnehmen. Für die Anregung der Fluoreszenzen in der festgelegten Wellenlänge ist immer nur ein Laser aktiv, der das Einwirken anderer Wellenlängen verhindert und mögliches „Durchbluten“ (engl. bleed through) ausschalten kann (sequentielle Aufnahme). Zudem bestrahlt der Laser nur einen kleinen Ausschnitt des Präparats, da eine kleine Lochblende (pin hole), die Aufnahme vor dem Ausbleichen schützt. Dies erhöht den Kontrast des gesamten aufgenommen Bildes. Ein weiterer großer Vorteil ist die Möglichkeit der wiederholten Aufnahme, die bei der Verwendung der Epifluoreszenzmikroskopie durch das Ausbleichen der Fluorophore nicht besteht.

Der entscheidende Vorteil des in dieser Studie verwendeten software-gesteuerten AxioImager mit ApoTome ist, dass nach der Aufnahme ein vollständiger zusammenhängender 3DBildstapel in allen aufgenommenen Kanälen vorliegt. In diesem kann während der Analyse virtuell mikroskopiert und quantitativ analysiert werden. Prinzipiell ist dies bei den neuesten konfokalen Mikroskopen auch der Fall, jedoch stand für diese Arbeit ein solches nicht zur Verfügung. Des weiterem ist beim Zusammensetzen der einzelnen Kacheln der Algorithmus der Software ein kritischer Punkt. Die in dieser Arbeit verwendete Software „Neurolucida“ ist einer der Marktführer auf dem Gebiet der Epifluoreszenzmikroskopie. Als Alternative wäre ein konfokales Leica SP2 in Frage gekommen, bei dem die einzelnen Kacheln per Hand manuell zusammengefügt hätten werden müssen. Insbesondere durch die Tatsache, dass es sich bei dieser Arbeit um eine Kolokalisationsstudie handelt, wurde die Priorität auf eine automatische und fehlerärmere Zusammenlagerung gelegt. Um die getätigten Aufnahmen mit dem ApoTome zu verifizieren, wurden für jede Färbung Einzelkacheln am Leica SP2 durchgeführt (Abb. 4.1). Es konnte durch die konfokalen Aufnahmen eindeutig gezeigt werden, dass es sich bei den beschriebenen Kolokalisation in dieser Studie zwischen dem PV-Antikörper, dem WFA-Signal und der Gad1-Sonde mit den PVcre/tdTomato-Zellen um technisch saubere Befunde handelt und sie nicht durch ein „Durchbluten“ der Farbkanäle in der Epifluoreszenzmikroskopie zustande gekommen sind (Abb. 4.1). 

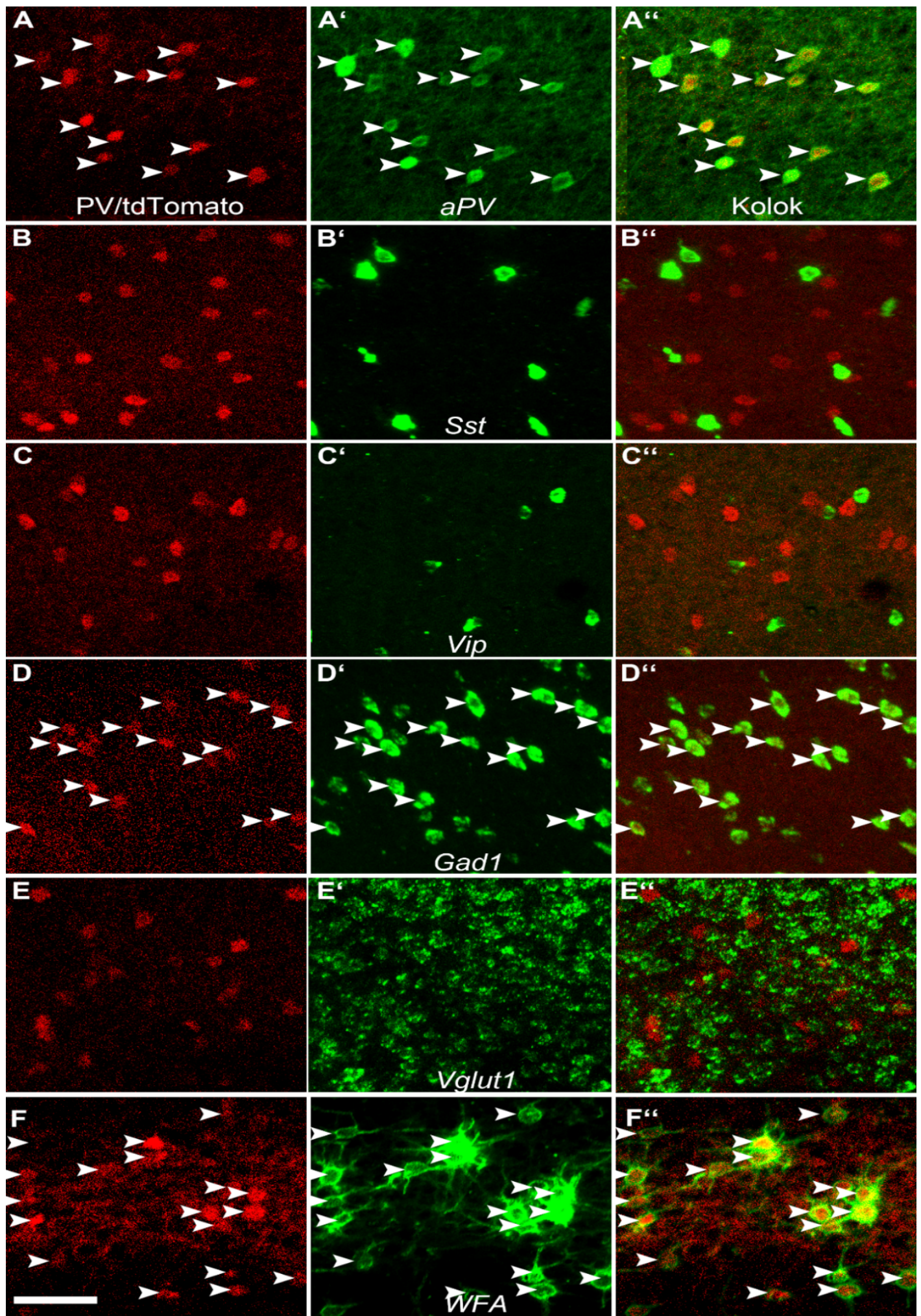

Abbildung 4.1: Konfokale Aufnahme der IHC und FISH aus Schicht IV

Die IHC des PV(Swant) (A-A') und des Antikörpers WFA (F-F") sowie die FISH der Sst- (B-B'), Vip- (C-C' $)$, Gad1- (D-D') und Vglut1-Sonde (E-E"') an frontalen Hirnschnitten der PVcre/tdTomato-Maus zeigt in Rot aus Schicht IV das transgene tdTomato-Signal (A-F), in Grün das Antikörper/Sonden-Signal $\left(\mathbf{A}^{\prime}-\mathbf{F}^{\prime}\right)$, deren Übereinanderlagerung und Kolokalisationen $\left(\mathbf{A}^{\prime \prime}-\mathbf{F}^{\prime \prime}\right)$ in Gelb. 


\subsubsection{Auszählung und manuelles Einzeichnen der Schichten}

Die Basis der schichtspezifischen Charakterisierung besteht in der Abgrenzung einzelner Schichten im primären somatosensorischen Kortex der PVcre/tdTomato-Maus. Die Schichtenabgrenzung erfolgte manuell anhand der Zellkernfärbung mit DAPI (blau), in der sich gut die Zelldichte erfassen lässt. Außen kann die Pia mater eingezeichnet werden. Die Schicht I lässt sich durch die geringere Zelldichte von der Schicht II/III gut abgrenzen. Die in der Schicht II/III gleichmäßig verteilten Zellen werden als eine einheitliche Schicht gewertet, da eine Separierung voneinander nicht möglich ist. Von ihr sondert sich die prägnante Schicht IV durch ihre dicht gepackten Zellen und der im primär somatosensorischen Kortex der Maus damit verbundenen Barrel-Struktur ab (Ren et al. 1992). Die Schicht V kann in Va und Vb unterteilt werden. Bekannt ist, dass Va ein Drittel der gesamten Schicht V ausmacht und von Vb durch seine erhöhte Zelldichte abzugrenzen ist. Zuletzt kann die Schicht VI anhand ihrer kleineren, gleichmäßig verteilten Zellen und ihrer höheren Zelldichte von Vb abgegrenzt werden (Schubert et al. 2006).

Insgesamt kann das manuelle Einzeichnen der Schichten als kritisch betrachtet werden und zum Teil zu verfälschten Ergebnissen führen. Durch den Vergleich der einzelnen Schichtgrößen der Schnitte untereinander und den vorgegebenen bekannten kortikalen Schichtdicken (DeFelipe et al. 2002), kann man jedoch abschätzen, ob das eigene Einzeichnen in der vorgegeben Norm liegt. In der Horizontalen wurde für die ausgezählten Schnitte eine einheitliche Breite von 1000 $\mu \mathrm{m}$ eingestellt. Eine schichtenunabhängige Analyse der Verteilung der PVcre/tdTomatopositiven Zellen und deren Kolokalisationen anhand einer Bin-size Analyse wurde innerhalb dieser Studie nicht durchgeführt. Bei der Bin-size Analyse wird der ausgewählte Bereich des Kortex von der Pia mater zur weißen Substanz, für jeden Schnitt auf $1000 \mu \mathrm{m}$ normiert und anschließend in 20 Bins (Bereiche gleicher Größe) von $50 \mu \mathrm{m}$ unterteilt. In der Analyse werden die Zellsomata aufgrund ihrer Distanz zur Pia mater in die jeweiligen Bins eingeteilt. Danach kann das Programm Neuroexplorer die gesamte Zelldichte für jeden Bin berechnen und eine schichtenunabhängige Auswertung erfolgen. In dieser Studie wird diese Methode allerdings nicht verwendet, da vergleichbare Versuche zeigen, dass eine manuelle schichtspezifische Auswertung anhand der beschriebenen Kriterien reproduzierbare und interpretierbare Ergebnisse erzielen (Scheuer 2015).

Weiterhin muss in der Auszählung bei Zellen, die auf einer eingezeichneten Schichtgrenze bzw. auf der Grenze des Auszählareales entschieden werden, zu welchem Gebiet sie gezählt werden. Hierfür wurde das $50 \%$ Kriterium angewendet. Zellen wurden in den Bereich zugeordnet, der 
mindestens $50 \%$ der Somafläche beeinhaltete. Dementsprechend wurde die Liniendicke vereinheitlicht und sehr dünn eingestellt. Bei der Bestimmung, ob es sich wirklich um den Zellkörper handelt, wurde der Farbkanal mit der Zellkernfärbung DAPI abgeglichen. Die Morphologie der Zelle wurde nicht berücksichtigt. Bei der Kolokalisationsuntersuchung der Antikörper bzw. Sonden mit dem nativen Rotsignal wurde immer eine Ja-Nein-Entscheidung durchgeführt. Die Schnitte wurden für den Experimentator blind ausgewertet, d. h. die Art der Färbung war nicht bekannt. In Einzelfällen wurden Schnitte durch unterschiedliche Personen ausgezählt, um die Reproduzierbarkeit der Ergebnisse zu überprüfen.

\subsection{Diskussion der Ergebnisse}

Inhibitorische GABAerge Interneurone können anhand ihrer Expression von neurochemischen Markern in Subtypen klassifizieren werden. Neben den SOM-expimierenden und den 5HT3a-Rezeptor exprimierenden Interneuronen stellen die Parvalbumin (PV)exprimierenden Interneurone mit ca. 40 \% die größte Gruppe dar (Rudy et al. 2011). Sie werden auch als Klasse der schnell feuernden, fast spiking Interneurone bezeichnet (Cauli et al. 1997). Morphologisch lassen sich die PV-exprimierenden Neurone in große Korbzellen, (LBCs, large basket cells), kleine Korbzellen (SBCs, small basket cells) und Nestkorbzellen (NBCs, nested basket cells) sowie die Gruppe der Axo-axonische bzw. Chandelierzellen (ChCs, chandelier cells) unterteilen (Markram et al. 2004; Tremblay et al. 2016). Eine Koexpression der neurochemischen Marker PV, Somatostatin und vasoaktive intestinale Polypeptid (VIP) im Bereich des somatosensorischen Kortex konnte einerseits ausgeschlossen (Kubota et al. 1994; Scheuer 2015) und andererseits gefunden werden (Lee et al. 2010). Diese Studie soll diesen kontroversen Befunden nachgehen und zudem das vermehrte Vorkommen von perineuronalen Netzen (PNN) an PV-exprimierenden Zellen im Gehirn belegen. Des Weiteren wurde die schichtenspezifische Verteilung der PV-exprimierenden Neurone genauer untersucht.

\subsubsection{PV-exprimierende Interneurone der PVcre-Maus}

In den Studien zur Untersuchung PV-exprimierender Interneurone bzw. schnell-feuernder Neurone wird überwiegend die transgene Parvalbumin-tdTomato-Maus verwendet, deren Ausgangspunkt die PVcre-Maus darstellt. Daher wurden in dieser Studie acht PVcre/tdTomatoMäuse für deren neurochemische Charakterisierung eingesetzt. 
$\mathrm{Zu}$ Beginn dieser wurde der Frage nachgegangen: Inwieweit die nativen rot fluoreszierenden Zellen im Bereich des Barrel-Kortex der PVcre/tdTomato-Maus das Calzium-bindende Protein Parvalbumin exprimieren. An sieben Hirnschnitten (4 Tiere) weisen 87,6 \% der tdTomatoZellen eine Kolokalisation mit dem PV-Antikörper auf und 95,6 \% der aPV-positiven Zellen sind tdTomato-positiv. In der schichtenspezifischen Analyse zeigen sich keine Unterschiede zwischen den einzelnen kortikalen Schichten, demzufolge sind die restlichen 12,4 \% der nichtPVcre/tdTomato-Zellen im gesamten Barrel-Kortex zu finden. Um welche Subtypen handelt es sich bei diesen Neuronen?

Um diese Frage zu beantworten, wurden die tdTomato-positiven Zellen hinsichtlich möglicher Kolokalisation mit den Interneuronsubpopulationenmarkern Somatostatin und vasoaktives intestinale Polypeptid überprüft. Hierfür wurden mittels Fluoreszenz-in-situ-Hybridisierung Technik jeweils acht Schnitte von vier Tieren mit der Vip- und Sst-Sonde behandelt. Bei der $V$ ip-Sonde konnten lediglich 20,8 \pm 27,0 kolokalisierte tdTomato-Zellen (Tab. 6.9) innerhalb des Barrel-Kortex beobachtet werden, das entspricht $\approx 0,2 \%$ der tdTomato-Zellen (Tab. 6.10.). Insgesamt verteilen sich diese Zellen lediglich auf Schicht II/III. Da grundsätzlich fast $60 \%$ aller VIP-exprimierenden Neurone in der Schicht II/III vorkommen (Prönneke et al. 2015), kann dies die erhöhte Wahrscheinlichkeit der Kolokalisation von tdTomato-Zellen bzw. Parvalbumin-exprimierenden Zellen mit der Vip-Sonde erklären. Letztlich kann festgehalten werden, dass die nicht-PVcre/tdTomato-positiven Zellen keine VIP-exprimierenden Interneurone darstellen.

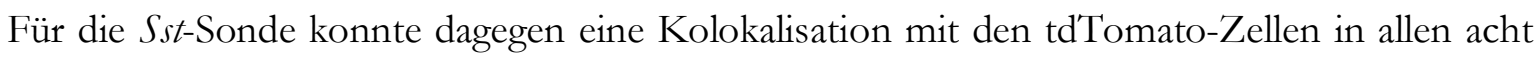
Hirnschnitten mit insgesamt 559,5 $\pm 245,2$ Zellen $/ \mathrm{mm}^{3}$ festgestellt werden. Das entspricht 5,3 $\pm 2,4 \%$ der tdTomato-Zellen. Die SOM-positiven tdTomato-Zellen sind vorrangig auf die infragranulären Schichten verteilt ( $\approx 60 \%$ ), bei der die Schicht Vb mit 166,7 \pm 97,2 SOMkolokalisierten tdTomato-Zellen hervorsticht. Die relativ hohe Überlappung des tdTomatoSignals mit der Sst-Sonde insbesondere in den Schichten Va und Vb kann aufgrund der präferentiellen Lage von SOM-positiven Neuronen mit über $47 \%$ in diesen Schichten begründet werden (Fischer 2018). Des Weiteren zeigen in der SOM-cre Maus knapp $40 \%$ der Schicht V SOM-positiven Neurone ein annäherndes schnell feuerndes Aktionspotentialmuster infolge überschwelliger Strominjektionen (Nigro et al. 2018). Ein Feuermuster welches ansonsten für die schnell-feuernden PV-Zellen charakteristisch ist. Dieser Befund konnte bereits 2013 von $\mathrm{Hu}$ und Kollegen in Hirnschnitten von somatosensorischem Kortex gefunden werden (Hu et al. 2013). Sie zeigten, dass 6-10 \% der SOM-positiven Neuronen Parvalbumin 
exprimieren und ein schnell-feuerndes Feuermuster aufweisen. Die Überlappung der PV und SOM Expression könnte auf die kurzzeitige SOM Expression in der embryonalen Entwicklung in einer kleinen Subpopulation von PV-Interneuronen zurückzuführen sein (Yavorska und Wehr 2016).

Hinsichtlich der Fragestellung was die tdTomato-Zellen ohne PV-Antikörperfärbung exprimieren, besteht die Möglichkeit, dass es sich bei ca. $30 \%$ der nicht PV-kolokalisierten Zellen und insbesondere bei den tdTomato-Zellen in den Schichten IV und V um Somatostatinpositive Zellen handelt. Um diese Frage weiter aufzuklären, besteht die Möglichkeit der Testung weiterer PVcre/tdTomato-Mäuse mit dem Antikörper gegen Somatostatin. Ob jedoch die IHC eine andere und eindeutigere Antwort liefern kann, darf bezweifelt werden. In vorangegangenen Charakterisierungsstudien des Labors lieferten die beiden Messmethoden IHC und FISH in ihren Ergebnissen größtenteils gleiche Verteilungsmuster und Kolokalisationen; für die VIP-cre Maus (Scheuer 2015). Zusammenfassend bestätigt die insgesamt geringe Kolokalisation (SOM bei PV/tdTomato) unter den Subpopulationen an GABAergen Interneuronen die zuvor aufgestellte Aussage, dass die drei molekularen Marker Parvalbumin, Somatostatin und VIP distinkte Subpopulationen von inhibitorischen Neuronen markieren (Markram et al. 2004; Xu et al. 2010; Rudy et al. 2011; Tremblay et al. 2016). Allerdings markieren diese drei genannten Marker nur ca. $80 \%$ aller GABAerger Interneurone (Xu et al. 2010). Die VIP-exprimierenden Neurone gehören zur heterogenen Gruppe des ionotropen Serotoninrezeptors 3a (5HT3aR) und machen dort nur ca. 40 \% aus (Rudy et al. 2011). Aus diesem Grund könnten die restlichen nicht mit PV-kolokalisierten Neurone zum molekularen Subtyp der 5HT3a-Rezeptor exprimierenden nicht VIP-positiven Zellen gehören. Zu dieser Gruppe zählen auch die neurogliaformen Zellen (NGFC). Diese Zellen unterscheiden sich aufgrund ihrer spezifischen axonalen Morphologie und differenzieren sich als eine separate Zellgruppe (Staiger et al. 2015). Um diese Annahme verfestigen zu können, müssten weitere Färbungen an der PVcre/tdTomato-Maus durchführen werden. Jedoch sind bislang wenige spezifische Marker weder für Non-VIP-Zellen noch für neurogliaforme Zellen verwendbar, die eine verlässliche Identifikation der beiden Interneuronentypen vornehmen könnten.

Eine letzte und bisher ausgeschlossene Möglichkeit besteht, dass es sich bei den nicht mit PVkolokalisierten tdTomato-Zellen um gar keine inhibitorischen, sondern um exzitatorische Zellen handelt. Dafür wurden jeweils acht Schnitte (vier Tiere) in der FISH mit dem exzitatorischen Marker Vglut und dem inhibitorischen Marker Gad1 untersucht. Die VglutSonde wird als Marker für exzitatorische Neurone angesehen, weil der Transporter Glutamat in 
synaptische Vesikel akkumuliert. Die Gad1-Sonde gilt als Marker für GABAerge Interneurone, da das Enzym bei der Katalyse Glutamat zu GABA umwandelt. Im Bereich des Barrel-Kortex weisen 92,1\% (Tab. 6.7) der tdTomato-Zellen eine Kolokalisation mit Gad1 auf. Daher kann den PVcre/tdTomato-Zellen eine überwiegende inhibitorische Eigenschaft zugeschrieben werden, so wie es bereits in der Literatur angegeben ist (Lee et al. 2010; Kubota et al. 1994). Die nicht mit der Gad1-Sonde kolokalisierten tdTomato-Zellen zeigen dabei eine Präferenz für die kortikalen Schichten $\mathrm{Va}$ und besonders für Schicht $\mathrm{Vb}$ (Abb. 3.5). Betrachtet man im Umkehrschluss die Ergebnisse für die Vglut-Sonde, so zeigt diese in den Schichten $\mathrm{Va}$ und $\mathrm{Vb}$ eine deutliche Kolokalisation (Schicht Va 6,1 \pm 4,71\% und Schicht Vb 18,22 \pm 6,57\%) mit den tdTomato-Zellen der PVcre-Maus (Abb. 3.7, Tab. 6.7). Diese beiden Ergebnisse gehen Hand in Hand und legen den Rückschluss nahe, dass es sich bei den nicht Gad1-kolokalisierten tdTomato-Zellen insbesondere in der Schicht $\mathrm{V}$ um exzitatorische ( $V g l u t$-positive) Zellen handelt. In einer Studie im PV1-Kern des Hypothalamus konnten Vglut2 positive Parvalbuminexprimierende Neurone beschrieben werden (Girard et al. 2011). Es ist derzeit noch unklar, ob PV-Zellen ähnliche Funktionen in verschiedenen sensorischen Regionen durchführen können. Angenommen wird jedoch, dass sie gezielt eine Verstärkung in kortikalen Netzwerken projizieren, indem sie lokale exzitatorische Inputs zusammenfügen und diese an andere PVZellen als auch an Pyramidenzellen weitergeben (Yavorska und Wehr 2016). In welche Richtung sich das Verhalten durch die Annahme der möglichen exzitatorischen Eigenschaft von PVexprimierenden Interneuronen ändert, müsste durch weitere Studien analysiert werden. Bisher gibt es zusätzlich noch ähnliche Ansätze erregender Eigenschaften von Interneuronen, die besagen, dass beispielsweise Axo-axonische-Zellen im Neokortex ein depolarisierendes Umkehrpotential besitzen und somit exzitatorische statt inhibitorische Reaktionen hervorrufen können (Szabadics et al. 2006). Eine andere und zutreffendere Erklärung ist, dass es sich bei den nicht Gad1-positiven rot fluoreszierenden tdTomato-Zellen in den Schichten $\mathrm{Va}$ und $\mathrm{Vb}$ um exzitatorische Pyramidenzellen handelt. Wie könnte es zur Markierung dieser Zellen kommen? Bei der embryonalen Herkunft erregender und hemmender Neuronen gibt es charakteristische Unterschiede. Exzitatorische Neurone entstehen aus neuronalen Vorläuferzellen in der Ventrikulärenzone des dorsalen Telenzephalons, während die inhibitorischen Neurone aus dem ventralen Telenzephalon stammen (Sakakibara und Hatanaka 2015). Kortikale GABAerge Interneurone wandern aus der medialen und caudalen ganglionäre Eminenz (MGE und CGE) und über den präoptischen Bereich (POA) Richtung dorsalem Telenzephalon (Lodato et al. 2011). Die Parvalbumin- und die Somatostatin-exprimierenden Interneuronen stammen primär aus der medialen Ganglien-Eminenz (MGE) des subkortikalen 
Telenzephalons (Xu et al. 2004). Sie wandern während der embryonalen Entwicklung wie alle GABAergen Zellen aus der ganglionalen Eminenz tangential zum Neokortex. In einigen Studien wurde gezeigt, dass GABAerge und Glutamaterge Neuronen bei gleichzeitiger Entstehung trotz ihrer tangentialen bzw. radialen Migration innerhalb ihrer neokortikalen Entwicklung voneinander abhängig sind (Tan und Shi 2013). Dies könnte dazu führen, dass die beiden Zelltypen sich untereinander beeinflussen und Ähnlichkeiten in ihren Eigenschaften aufweisen. So könnte in der PVcre-Maus, in der die Cre-Rekombinase eingebracht wurde, diese auch in einigen wenigen Pyramidenzellen exprimiert werden. Dadurch könnten in der PVcre/tdTomato-Maus ebenfalls Pyramidenzellen das rot fluoreszierende tdTomato-Signal tragen.

Nicht außer Acht lassen, sollte man die verminderte Bindung von Sonden an die jeweilige mRNA im Vergleich zu einem Antikörper an ein bestimmtes Protein. Diese Beobachtung konnte bereits in anderen Charakterisierungsstudien von transgenen Mäusen im Labor der Arbeitsgruppe gezeigt werden (Scheuer 2015; Fischer 2018). Auch spricht das schichtenspezifische Auftreten der Kolokalisation bzw. Nicht-Kolokalisation gegen diese Möglichkeit. Eine zur FISH korrespondierende Antikörperfärbung zum Nachweis der inhibitorischen und exzitatorischen Eigenschaften der tdTomato-Zellen konnte nicht durchgeführt werden. Einerseits ist derzeit kein geeigneter Antikörper zur Markierung inhibitorischer Zellen verfügbar, der ein zufriedenstellendes Ergebnis erzielt (z. B. anti-GABA Färbung). Andererseits wurde auf die Verwendung eines Antikörpers gegenüber exzitatorischer Neurone verzichtet, da diese vermehrt die synaptischen Boutons, also die Axon-terminale im Kortex markieren, und keine Kolokalisationsstudien mit einem zytosolischen vorkommenden Fluorophor ermöglicht. Kurz erwähnt werden sollte die Möglichkeit, dass inhibitorische Zellen die nicht Gad1-positiv sind, die Isoform2 des Enzyms (GAD2) exprimieren. GAD2 wird ebenfalls zur Identifizierung GABAerger Interneurone eingesetzt (Besser et al. 2015). Im Rahmen dieser Studie war eine Markierung mit der GAD2-Sonde nicht möglich. Ungeachtet dieser Problematik ist die starke Kongruenz zwischen der Gad1-Sonde und der Vglut-Sonde offensichtlich, welche klar für eine Markierung exzitatorischer Zellen in der Schicht V des Bartel-Kortex spricht.

Zusammenfassend kann für die PVcre-Maus festgehalten werden, dass lediglich knapp 88 \% der tdTomato-positiven Zellen den Marker Parvalbumin exprimieren und demnach zur Klasse der PV-exprimierenden Interneurone gezählt werden können. Bei den restlichen tdTomatoZellen handelt es sich insbesondere in den kortikalen Schichten $\mathrm{Va}$ und $\mathrm{Vb}$ um exzitatorische 
Pyramidenzellen. Des Weiteren könnten Somatostatin-exprimierende Interneurone unter den tdTomato-positiven Zellen sein, die sich überwiegend auf die Schichten IV und V verteilen. Letztlich kann nicht ausgeschlossen werden, dass insbesondere in den supragranulären Schichten ebenso nicht-VIP 5HT3a-Rezeptor exprimierende Interneurone mit dem tdTomatoSignal in der PVcre-Maus markiert sind. Aus diesem Grund kann die PVcre-Maus nicht als spezifisches transgenes Tiermodell für Parvalbumin-exprimierende Interneurone angesehen werden. Sie ist für klassische elektrophysiologische Untersuchungen, z. B. patch-clamp Abletungen, geeignet, bei der eine weitere Charakterisierung erfolgen kann, z. B. Aktionspotentialfeuermuster. Hingegen ist bei optogenetischen und Tracing-Studien, die die Schicht $\mathrm{V}$ beinhalten darauf $\mathrm{zu}$ achten, die erhobenen Ergebnisse hinsichtlich einer Kontamination durch exzitatorische Pyramidenzellen zu überprüfen. Diese „Verunreinigung“ der Linie kann zu Fehlinterpretationen und letztlich zu falschen Schlussfolgerungen führen. Insbesondere bei Konnektivitätsstudien im Gehirn bei denen die Tracer über gentechnisch veränderte Viren (Cre-abhängige AAV oder nicht-pathogene Tollwutviren) eingebracht werden, ist die PVcre-Maus nicht verwendbar.

\subsubsection{Verteilung der tdTomato-Zellen in der PVcre-Maus}

Unabhängig von der Spezifität als Marker für PV-exprimierende Interneurone wurde die Zellverteilung der nativen rot fluoreszierenden tdTomato-Zellen innerhalb der Schichten des Barrel-Kortex untersucht. Hierfür wurden 47 Schnitte von acht Tieren aus den vorangegangenen immunhistochemischen Färbung und der Fluoreszenz-in-situ-Hybridisierung herangezogen. In der schichtenspezifischen Auswertung zeigte sich eine starke Präferenz der tdTomato-Zellen auf die Schichten IV (28,7 \%) und Vb (24,7 \%) (Tab. 6.4). Damit sind ca. 53 $\%$ der tdTomato-Zellen in der Haupteingangs- bzw. Ausgangsschicht des Barrel-Kortex lokalisiert. Dies lässt auf ein gerichtetes schichtenspezifisches Aufgabenprofil der im weitestgehend PV-exprimierenden Interneurone schließen. Die Schicht IV wird in derzeitigen Modellvorstellungen immer noch als zentraler Ausgangspunkt kortikaler Informationsverarbeitung angesehen, von der eine Vielfalt an sequentiell oder parallel organisierten synaptischen Netzwerken beginnen (Petersen 2007; Schubert et al. 2007; Staiger et al. 2009). Bereits vorangegangene Studien der Arbeitsgruppe konnten Hinweise auf ein vermehrtes Vorkommen der Parvalbumin-exprimierenden Zellen in den Schichten IV und Vb finden (Scheuer 2015). Die Rückschlüsse waren Seitenbefunde anhand Antikörperfärbungen gegen das Calzium-bindende Protein Parvalbumin bzw. FISH gegen die PV-mRNA. Ebenfalls 
eine Studie an Ratten zeigte, dass die meisten Parvalbumin-exprimierende Interneuronen in der Schicht IV lokalisiert sind (Staiger et al. 2009). Zusätzlich besitzen PV-exprimierenden Interneuronen eine Aufgabe im inhibitorischen Mikroschaltkreis, d. h. sie fungieren nicht nur als reine feedforward-Inhibitoren der exzitatoischen Zellen im Kortex, sondern projizieren auf ein weiteres GABAerges Interneuron und lösen dadurch ein Ausbleiben der Inhibition auf ein Prinzipalneuron aus, auch Disinhibition genannt (Staiger et al. 2015). In neueren Arbeiten wurde ihre Rolle als Disinhibitoren für ihre eigene Subpopulation und auf SOM-exprimierende Neurone, insbesondere Martinotti-Zellen gezeigt (Pfeffer et al. 2013; Walker et al. 2016). Ihre dritte Aufgabe ist außerdem die Feedback-Inhibition, also rückwärtsgerichtete Hemmung, indem sie ihre Stimulation auf die gleiche Hauptzelle (reziproke Hemmung) oder eine ähnlichen Typs der Hauptzellpopulation (laterale Hemmung) überträgt (Feldmeyer et al. 2018). Ebenfalls erhalten die PV-exprimierenden Interneurone, insbesondere in den Schichten IV und Vb direkte thalamische Eingänge (Feldmeyer et al. 2018). Auf welchem dieser genannten Wege PVexprimierende Interneurone die kortikale Informationsverarbeitung in der Schicht IV und $\mathrm{Vb}$ beeinflussen, werden zukünftige Studien aufzeigen.

In der Schicht I $(0,1 \%)$ konnten nur in neun von 47 Hirnschnitten tdTomato-positive Zellen gefunden werden. Dieses geringe Vorkommen von vermutlich PV-exprimierenden Interneuronen konnte ebenfalls für den primär visuellen Kortex in anderen Studien gezeigt werden (Pfeffer et al. 2013). Die restlichen ca. $46 \%$ der tdTomato-Zellen sind mit 16,2\% auf die Schicht II/III, mit 15,1 \% auf die Schicht VI und mit 15,1 \% auf Schicht Va verteilt. Hier zeigt sich eine gleichmäßige Verteilung an tdTomato-Zellen. Grundlegend können die PVexprimierenden Interneuronen unterschiedliche Aufgabenprofile besitzen, die evtl. auch von Schicht zu Schicht varieren könnten. So ist vorstellbar, dass PV-exprimierende Interneurone in der Schicht IV überwiegend an einer Feedforward-Inhibition von thalamokortikalen Eingängen beteiligt sind und Schicht II/III PV-Zellen dagegen eine überwiegend disinhibitorische Wirkung besitzen. Das in dieser Studie aufgezeigte Verteilungsmuster der PVcre/tdTomato-Zellen sowie deren Kolokalisationen mit inhibitorischen Zellmarkern und insbesondere die Unspezifität der PVcre-Maus in der Schicht Vb für PV-exprimierene Interneurone werden als Grundlage anderer Forschungsarbeiten dienen, und darüber die Funktion von PV-exprimierenden GABAergen Neuronen im generellen und für die einzelnen kortikalen Schichten untersuchen. 


\subsubsection{PVcre/tdTomato-Zellen und perineuronale Netze}

Die PV-exprimierende Zellen werden hauptsächlich als schnell-feuernde Interneurone angesehen (Cauli et al. 1997). Für die Aufrechterhaltung der hohen Frequenz von Aktionspotentialen und der damit verbundenen synaptischen Neurotransmitterausschüttung wird eine hohe Stoffwechselaktivität dieser Zellen angenommen. Perineuronale Netze (PNN) sollen, insbesondere zum Schutz der Zellen vor oxidativem Stress, an diesen Mitochondrienreichen Zellen vorkommen (Cabungcal et al. 2013).

Um dieser Aussage nachzugehen wurden die PNN in der extrazellulären Matrix des BarrelKortex der PVcre/tdTomato-Maus mittels des Marker Wisteria floribunda Agglutinin (WFA) histochemisch angefärbt. In acht ausgezählten Hirnschnitten sind knapp 71 \% der tdTomatoZellen mit WFA kolokalisiert (Abb. 3.16, Tab. 6.13). In der schichtenspezifischen Analyse wurde eine hohe Heterogenität zwischen den Schichten deutlich und zeigte für die Schicht IV eine Kolokalisationsquote von 92,1 \% (Abb. 3.16). Aus der Tatsache begründet, dass nicht alle tdTomato-Zellen für Parvalbumin positiv sind und daher nicht zur Gruppe der PVexprimierenden Interneurone gezählt werden können (Abs. 4.2.1), wurde in der Analyse die Gegenprobe durchgeführt. Dabei konnte gezeigt werden, dass 99,2 \% der WFA-markierten Zellen das native tdTomato-Signal tragen (Tab. 6.13). Damit ist fast jede WFA-positive Zelle mit tdTomato markiert und legt die Vermutung nahe, dass viele PV-exprimierende Interneurone PNN in ihrer extrazellulären Matrix besitzen. Diese Aussage korreliert mit Studien an Ratten, in denen perineuronale Netze besonders mit den GABAergen inhibitorischen Neuronen assoziiert sind, die Parvalbumin enthalten (Kosaka und Heizmann 1989; Yamada und Jinno 2013). Die Aufgaben der Perineuronalen Netze sind bislang noch nicht eindeutig geklärt. Es besteht die Annahme, dass sie als Schutz vor verschiedenen neurodegenerativen Krankheiten dienen (Karetko und Skangiel-Kramska 2009). Des Weiteren werden sie als wichtiger Regulator der ZNS-Plastizität beschrieben, sowohl in der postnatalen Entwicklung wie auch im Erwachsenenalter (Wang und Fawcett 2012). Sobald man die PNNs in ihren Mechanismen grundlegend verstanden hat, gäbe es vielleicht eine Möglichkeit auf die Regulation neuronaler Plastizität Einfluss zu nehmen. Ein anderer Aspekt ist die weitreichende Konsequenz, die bei fehlerhafter Entwicklung der Perineuronalen Netze entstehen kann. Nicht nur Alzheimer, Epilepsie, Multiple Sklerose und Schlaganfall konnten mit PNN in Verbindung gebracht werden, sondern auch die Schizophrenie. Es bestehen Studien, die besagen, dass speziell die Schizophrenie mit Defiziten in PV-Neuronen assoziiert ist (Bitanihirwe und Woo 2014). Bei der Untersuchung der Schizophrenie wurden die PV-exprimierenden Neuronen als Hauptneuronenpopulation des Thalamuskern mit PNN analysiert und es wurde festgestellt, 
dass eine Veränderung in Form der Abnahme von PV-Neuronen mit PNN als Folge von oxidativem Stress im frühen Krankheitsverlauf vorliegt (Steullet et al. 2018). Ein weiteres Beispiel der Auswirkungen von fehlenden PNN im Kortex stellt die Epilepsie dar. Ein Abbau der PNN um schnell-feuernden PV-Zellen kann bei Hirntumorpatienten zu epileptischen Anfällen führen, da dieser Zustand zu einer niedrigeren Membrankapazität und somit zu schnelleren Aktionspotentialen kommen kann (Tewari et al. 2018).

Um verschiedene therapeutische Ansätze finden zu können, muss der Zusammenhang und die anzunehmende gegenseitige Abhängigkeit zwischen PNN und PV-exprimierenden Interneuronen in Bezug auf Zellentwicklung sowie daraus resultierende Krankheiten verstanden werden.

Jedoch scheint es auch PV-Interneurone zu geben, die keine PNN besitzen, insbesondere in den Schichten II/III, Va und VI, wo lediglich 64-70 \% der tdTomato-Zellen eine Markierung mit WFA zeigen und die Schicht Vb mit lediglich der Hälfte an Zellen im Vergleich zu Schicht IV (Tab. 6.13.). Es stellt sich die Frage, welcher Unterschied zwischen PNN-umhüllten PVNeuronen und PV-Neuronen ohne PNN besteht. Es wird angenommen, dass im somatosensorischen Kortex die Bildung der Perineuronalen Netze mit der kritischen Periodenschließung übereinstimmt (Pizzorusso et al. 2002). Jüngste Forschungen versuchen die Mechanismen zu verstehen, die die Einschränkung der ZNS-Plastizität durch perineuronale Netze erklärt (Wang und Fawcett 2012). Dieses Wissen wirft die Frage auf, ob die PV-Neurone ohne PNN ihre Plastizität in gleichem Maße beibehalten würden und inwieweit ihre Anzahl aktivitätsabhängige Auswirkungen auf neuronale Verbindungen im Gehirn hat.

\subsection{Relevanz der Studie}

Das Ziel der vorliegenden Studie ist die Charakterisierung der PVcre/tdTomato-Mauslinie hinsichtlich der Ko-Expression des tdTomato-Signals mit spezifischen Markern für GABAerge Interneurone. Es sollte vor allem geprüft werden, ob die nativen rot fluoreszierenden Zellen in der transgenen PVcre/tdTomato-Maus spezifisch für PV-exprimierende Interneurone sind und als eine verlässliche Basis für weiterführende Experimente angesehen werden können.

Die Ergebnisse dieser Studie machen überraschend deutlich, dass es sich bei der PVcreMauslinie um keine spezifische für PV-exprimierende Interneurone handelt. Zwar exprimieren 87,6 \% der tdTomato-Zellen Parvalbumin, jedoch gibt es in allen kortikalen Schichten auch nicht-PV-positive Zellen. Insbesondere in den tiefen kortikalen Schichten Va und Vb weist ein signifikanter Anteil dieser Zellen den exzitatorischen Marker Vglut1 auf. Daher befinden sich in 
diesen Schichten PV-Interneurone, die ebenfalls als exzitatorische Pyramidenzellen angesehen werden können. Dieses Wissen ist besonders bei der Verwendung der Maus in Cre-abhängigen Versuchen, z. B. Injektion von optogenetischen viralen Vektoren und in Tracing-Studien, von großer Bedeutung. Speziell für tracing Studien, in denen die Kontrolle der Starterzellen essenziell ist, um Aussagen über die Konnektivität einer Zellpopulation oder einer Region zu machen, ist von der PVcre abzuraten. Bisher durchgeführte Tracing-Studien sollten aufgrund der hier gezeigten Ergebnisse einer Prüfung unterzogen werden, um die allgemeine Gültigkeit der getroffenen Aussagen zu überprüfen. Natürlich sind nicht alle Ergebnisse von Studien mit der PVcre-Maus als „falsch“ zu bewerten, jedoch sollten sie kritisch beleuchtet werden. Optogenetische Studien, die sich mit der Konnektivität von PV-exprimierenden Interneuronen befassen und ausschließlich inhibitorische Ströme an den Zielzellen beobachteten sind, sollten ihre Aussagekraft beibehalten (Walker et al. 2016).

Zur Untersuchung von PV-exprimierenden Interneuronen unter in vitro Bedingungen scheint die PVcre-Maus dennoch geeignet zu sein. Die geringe Kolokalisation mit Somatostatin (5,3\%) in den Schichten II-VI, die vernachlässigbare $(0,2 \%)$ Kolokalisation mit VIP und die mögliche geringe tdTomato-Markierung von nicht VIP-positiven 5HT3a-Rezeptor exprimierenden Interneuronen, insbesondere in Schicht II/III, sprechen freilich gegen eine unkritische Verwendung. Jedoch kann in in vitro Ableitungen das charakteristische schnell-feuernde Aktionspotentialmuster als ein eindeutig zuzuordnendes Merkmal von PV-exprimierenden Zellen genutzt werden. Mit dieser Kenntnis sollten Studien mit der PVcre-Maus stets mit einer elektrophysiologischen Charakterisierung der abgeleiteten tdTomato-positiven Zellen beginnen. Anhand dieser können Daten von nicht PV-exprimierenden schnell-feuernden Interneuronen ausgeschlossen werden und präzisere Befunde erzielt und eindeutigere Rückschlüsse getroffen werden.

In einem weiteren Aspekt der Charakterisierung der PVcre/tdTomato-Mauslinie konnte das schichtspezifische Verteilungsmuster der nativen rot fluoreszierenden tdTomato-Zellen erhoben werden. Dabei zeigte sich ein bevorzugtes Vorkommen der tdTomato-Zellen in den Schichten IV und V mit mehr als 68 \%. Die Schicht IV enthält die meisten (28,7\%) tdTomatoZellen und wird als die Haupteingangsschicht der sensiblen Information von der Peripherie über den Thalamus angesehen (Petersen 2007). Des Weiteren bekommen PV-Interneurone selbst einen direkten thalamischen Eingang und sind maßgeblich in das feed-forward Inhibitionsnetzwerk eingebunden (Gabernet et al. 2005; Cruikshank et al. 2007; Kimura et al. 2010; Walker et al. 2016; Cruikshank et al. 2010; Staiger et al. 1996). Demzufolge wird den PV- 
Neuronen der Schicht IV eine entscheidende Rolle bei der Prozessierung somatosensorischer Information zugesprochen (Feldmeyer et al. 2013). Neben den thalamischen Projektionen in die Schicht IV über den lemniskalen Pfad existiert eine weitere starke Projektion vom Thalamus in den Kortex, der paralemniskale Pfad. Ausgehend vom posterioren medialen Kern des Thalamus (POM) terminieren die Axone überwiegend in den Schichten I und Va des somatosensorischen Kortex (Petersen 2007). Ebenfalls in dieser Eingangsschicht des BarrelKortex lassen sich mit gut $20 \%$ viele tdTomato-positive Zellen nachweisen. Obwohl in der infragranulären Schicht V ebenfalls exzitatorische Pyramidenzellen mit tdTomato markiert sind, ist von einer großen Population von PV-exprimierenden Interneuronen auszugehen. Daher könnte man sich analog zu Schicht IV ein Verschaltungsschema der PV-Interneurone nach dem feed-forward Inhibitionsprinzip vorstellen. Obwohl der paralemniskalen Projektion keine große Beteiligung an der somatosensorischen Verarbeitung zugesprochen wird (Lavallée et al. 2005), scheint sie eine wichtige Rolle bei der aktiven Erkundung und sensomorischer Koordination zu spielen (Petersen 2007). In wieweit PV-Interneurone in der Schicht Va in das kortikale Netzwerk eingebunden sind, ist nur ansatzweise untersucht und bedarf weiterer Konnektivitätsstudien.

Neben der hohen Anzahl an PV-Interneuronen in der kortikalen Haupteingangsschicht IV kommen in der Hauptausgangsschicht des Kortex, Schicht Vb, mit 24,7 \% ebenfalls eine große Population von tdTomato-Zellen vor. Nach Abzug der tdTomato-markierten exzitatorischen Pyramidenzellen (ca. 18 \%) sollten auch in der Schicht Vb im Vergleich zu den Schichten II/III und VI mehr PV-Interneurone vorkommen. Die Mehrzahl der PV-exprimierenden Interneurone werden zur Subgruppe der Korbzellen gezählt (Markram et al. 2004; Tremblay et al. 2016). Charakteristisch für diesen morphologischen Subtyp ist die Ausbildung von korbähnlichen Synapsen um die Somata bzw. proximale Dendriten ihrer Zielzellen. Damit sind sie in der Lage die Generierung von Aktionspotentialen nahe ihrer Entstehung zu hemmen und damit die Informationsweiterleitung $\mathrm{zu}$ blockieren. PV-Interneurone sind einerseits schichtenunabhängig mit PV-Interneuronen verbunden und andererseits projizieren Schicht V PV-Interneurone auf Schicht V Pyramidenzellen (Pfeffer et al. 2013; Jiang et al. 2015).

Letztere Konnektivität spricht für die Möglichkeit, dass PV-Interneurone der Schicht Vb in der Lage wären den kortikalen Output über Pyramidenzellen zu modulieren und zu kontrollieren. 


\section{$5 \quad$ Zusammenfassung}

Die Neurone des primären somatosensorischen Kortex der Maus können in inhibitorische und exzitatorische Populationen eingeteilt werden. Die Gruppe der inhibitorischen Interneurone, die ca. 15-20 \% der Neurone umfassen, setzen den Neurotransmitter GABA frei und werden als GABAerge Neuronen bezeichnet. Sie können aufgrund ihrer elektrophysiologischen, morphologischen und molekularen Eigenschaften weiter in Subpopulationen unterteilt werden. Im Hinblick auf die Expression von Parvalbumin (PV), Somatostation (SOM) und des ionotropen Serotonin Rezeptors 5HT3a lassen sich drei molekular distinkte Subpopulationen von GABAergen Neuronen abgrenzen. Die 5HT3aR-Population, wird zudem in die Subgruppe vasoaktivem intestinalem Peptid VIP- (vasoaktives intestinales Polypeptid) (40\%) und Nicht-VIPexprimierende Zellen (60\%) unterteilt.

In der vorliegenden Studie wurde die durch die Cre/loxP-Technik generierte transgene PVcre/tdTomato-Maus verwendet, in der PV-exprimierende GABAerge Neurone das rot fluoreszierende tdTomato-Protein tragen. In dieser Arbeit sollte die PVcre-Mauslinie mittels Immunhistochemie und Fluoreszenz-in-situ-Hybridisierung im somatosensorischen Kortex (Barrel-Kortex) charakterisiert werden. Die tdTomato-positiven Zellen wurden hinsichtlich der Expression spezifischer Interneuronmarker wie Parvalbumin (PV), Somatostatin (SOM) und vasoaktiven intestinalen Polypeptid (VIP) untersucht. Zusätzlich werden die rot fluoreszierenden Zellen auf ihre inhibitorische Eigenschaft (Marker Gad1) geprüft sowie die Gegenprobe mit dem exzitatorischen Marker Vglut1 durchgeführt. Des Weiteren sollte untersucht werden, inwieweit perineuronale Netze (Marker WFA) an den PV-exprimierenden Neuronen vorkommen. Das Ziel dieser Studie ist es Aussagen über die schichtenspezifische Zellverteilung der tdTomato-positiven Zellen sowie die Spezifität des tdTomato-Signals für PVexprimierenden GABAerge Neurone im Barrel-Kortex zu treffen. In der schichtenspezifischen Analyse der PVcre/tdTomato-Mauslinie im Barrel-Kortex zeigte sich eine starke Präferenz der tdTomato-Zellen auf die Schichten IV (28,7 \%) und Vb (24,7\%). Dabei konnte lediglich für 92,1 \% der Zellen eine Kolokalisation mit dem inhibitorischen Marker Gad1 festgestellt werden. Insbesondere in den kortikalen Schichten $\mathrm{Va}$ und $\mathrm{Vb}$ wiesen ca. $7 \%(\mathrm{Va})$ und $18 \%(\mathrm{Vb})$ der tdTomato-Zellen eine Ko-Expression mit Vglut1 auf und besitzen demnach exzitatorische Eigenschaften. In der Auswertung der Marker für GABAerge Neurone exprimieren 87,6 \% der tdTomato-Zellen Parvalbumin und können somit als PV-exprimierende GABAerge Neurone angesehen werden. Eine geringe Anzahl der tdTomato-Zellen exprimiert das Neuropeptid Somatostatin (5,3\%), insbesondere in den Schichten Vb und VI. Dagegen konnte eine Kolokalisation mit der Vip-Sonde nahezu ausgeschlossen werden (0,2 \%). In der 
PVcre/tdTomato-Maus trugen 70,9\% der tdTomato-Zellen im Barrel-Kortex perineuronale Netze, dabei war deren Überlappung mit 92,1 \% in der Schicht IV am höchsten. Interessanterweise sind 99,2\% aller PNN tragender Zellen durch das tdTomato-Signal markiert. Zusammenfassend konnte in dieser Arbeit nachgewiesen werden, dass die PVcre-Maus kein spezifisches Mausmodell für PV-exprimierende GABAerge Neurone darstellt. Insbesondere die hohe Anzahl an markierten exzitatorischen Neuronen in der Schicht V sollte bei den Nutzern der Linie Beachtung finden. Es wird angeraten weitere Carakterisierungsmethoden anzuwenden, um verlässliche und reproduzierbare Ergebnisse für diese Mauslinie zu erhalten. 


\section{Anhang}

Tabelle 6.1: Anzahl der tdTomato-Zellen und der aPV-positiven Zellen in den einzelnen Schichten des Barrel-Kortex

\begin{tabular}{|c|c|c|c|c|c|}
\hline Region & alle tdTomato & nur tdTomato & alle aPV & aPV & Kolok \\
\hline Barrel-Kortex & $8943,5 \pm 383,6$ & $1097,0 \pm 561,8$ & $8194,9 \pm 648,5$ & $348,4 \pm 279,2$ & $7846,6 \pm 816,0$ \\
\hline Layer I & $3,1 \pm 8,2$ & $0,0 \pm 0,0$ & $3,1 \pm 8,2$ & $0,0 \pm 0,0$ & $3,1 \pm 7,6$ \\
\hline Layer II/III & $1514,7 \pm 163,8$ & $145,9 \pm 138,1$ & $1424,5 \pm 104,1$ & $55,7 \pm 62,9$ & $1368,8 \pm 107,8$ \\
\hline Layer IV & $2617,4 \pm 297,9$ & $259,6 \pm 213,1$ & $2445,5 \pm 394,4$ & $87,7 \pm 76,3$ & $2357,7 \pm 364,9$ \\
\hline Layer Va & $1383,0 \pm 169,5$ & $152,1 \pm 76,4$ & $1255,6 \pm 177,4$ & $24,6 \pm 40,1$ & $1230,9 \pm 139,3$ \\
\hline Layer Vb & $2297,8 \pm 235,2$ & $423,0 \pm 187,3$ & $1971,8 \pm 347,2$ & $97,0 \pm 103,8$ & $1874,8 \pm 336,2$ \\
\hline Layer V (a+b) & $3680,8 \pm 245,8$ & $575,1 \pm 252,7$ & $3227,4 \pm 354,7$ & $121,6 \pm 101,1$ & $3105,7 \pm 366,5$ \\
\hline Layer VI & $1124,2 \pm 278,5$ & $109,6 \pm 114,9$ & $1097,9 \pm 259,0$ & $83,3 \pm 79,4$ & $1014,6 \pm 276,8$ \\
\hline
\end{tabular}

Tabellarische Darstellung der Anzahl der kolokalisierten tdTomato-Zellen, nicht-kolokalisierten nur das native tdTomato-Signal oder nur das PV-Antikörper Signal tragende Zellen innerhalb der einzelnen Schichten für $1 \mathrm{~mm}^{3}$ Barrel-Kortex. Die Werte sind in Mittelwerte \pm Standardabweichung angegeben.

Tabelle 6.2: Prozentuale schichtspezifische Verteilung der Zellen, die nur tdTomato-positiv sind oder nur den Antikörper PV tragen sowie die kolokalisierten Zellen

\begin{tabular}{|c|c|c|c|}
\hline Region & nur tdTomato & nur aPV & Kolok \\
\hline Barrel-Kortex & $100,0 \pm 6,3$ & $100,0 \pm 80,2$ & $100,0 \pm 10,4$ \\
\hline Layer I & $0,0 \pm 0,0$ & $0,0 \pm 0,0$ & $0,0 \pm 0,1$ \\
\hline Layer II /III & $13,3 \pm 1,5$ & $16,0 \pm 18,0$ & $17,4 \pm 1,4$ \\
\hline Layer IV & $23,7 \pm 2,4$ & $25,2 \pm 21,9$ & $30,0 \pm 4,7$ \\
\hline Layer Va & $13,9 \pm 0,9$ & $7,1 \pm 11,5$ & $15,7 \pm 1,8$ \\
\hline Layer Vb & $38,6 \pm 2,1$ & $27,9 \pm 29,8$ & $23,9 \pm 4,3$ \\
\hline Layer V (a+b) & $52,4 \pm 2,8$ & $34,9 \pm 29,0$ & $39,6 \pm 4,7$ \\
\hline Layer VI & $10,0 \pm 1,3$ & $23,9 \pm 22,8$ & $12,9 \pm 3,5$ \\
\hline
\end{tabular}

Tabellarische Darstellung der prozentualen Verteilung von nur tdTomato-Zellen, nur aPV-positiver und deren Kolokalisation innerhalb der einzelnen Schichten für $1 \mathrm{~mm}^{3}$ Barrel-Kortex. Die Werte sind in Mittelwerte \pm Standardabweichung angegeben. 
Tabelle 6.3: Schichtspezifische Verteilung der kolokalisierten tdTomato-Zellen mit aPV

\begin{tabular}{|c|c|c|}
\hline & tdTomato mit aPV kolokalisiert & aPV mit tdTomato kolokalisiert \\
\hline Region & Kolokalisation & Kolokalisation \\
\hline Barrel-Kortex & $87,6 \pm 6,7$ & $95,6 \pm 3,7$ \\
\hline Layer I & $100,0 \pm 0,0$ & $100,0 \pm 0,0$ \\
\hline Layer II /III & $90,8 \pm 8,0$ & $96,1 \pm 4,4$ \\
\hline Layer IV & $89,8 \pm 9,0$ & $96,4 \pm 3,0$ \\
\hline Layer Va & $89,09 \pm 5,4$ & $98,2 \pm 2,6$ \\
\hline Layer Vb & $81,2 \pm 8,3$ & $95,1 \pm 5,3$ \\
\hline Layer V $\mathbf{( a + b )}$ & $84,2 \pm 7,0$ & $96,1 \pm 7,0$ \\
\hline Layer VI & $90,1 \pm 11,1$ & $91,7 \pm 8,0$ \\
\hline
\end{tabular}

Tabellarische Darstellung der mit dem aPV kolokalisierten tdTomato-Zellen bzw. den aPV-positiven Zellen mit dem tdTomato-Signal. Die prozentualen Werte sind jeweils für $1 \mathrm{~mm}^{3}$ Barrel-Kortex und für die einzelnen Schichten in Mittelwerte \pm Standardabweichung angegeben.

Tabelle 6.4: Prozentuale schichtspezifische Verteilung und die gesamte Anzahl an tdTomatoZellen

\begin{tabular}{|c|c|c|}
\hline Region & Alle tdTomato-Zellen in \% & alle tdTomato \\
\hline Barrel-Kortex & - & $9989,5 \pm 849,6$ \\
\hline Layer I & 0,1 & $5,6 \pm 12,5$ \\
\hline Layer II /II & 16,2 & $1617,3 \pm 207,7$ \\
\hline Layer IV & 28,7 & $2857,6 \pm 279,6$ \\
\hline Layer Va & 15,1 & $1499,8 \pm 220,8$ \\
\hline Layer Vb & 24,7 & $2468,8 \pm 403,7$ \\
\hline Layer V (a+b) & 39,7 & $3968,6 \pm 471,2$ \\
\hline Layer VI & 15,1 & $1522,8 \pm 349,4$ \\
\hline
\end{tabular}

Tabellarische Darstellung der prozentualen Verteilung der tdTomato-Zellen sowie die gesamte Verteilung der tdTomato-Zellen von allen 47 Schnitten innerhalb der einzelnen Schichten für $1 \mathrm{~mm}^{3}$ Barrel-Kortex. Die Werte sind in Mittelwerte \pm Standardabweichung angegeben. 
Tabelle 6.5: Anzahl der tdTomato-Zellen und der Gad1-positiven Zellen in den einzelnen Schichten des Barrel-Kortex

\begin{tabular}{|c|c|c|c|}
\hline Region & alle tdTomato & tdTomato & Kolok mit Gad1 \\
\hline Barrel-Kortex & $10540,7 \pm 822,4$ & $839,3 \pm 342,4$ & $9701,4 \pm 807,2$ \\
\hline Layer I & $17,9 \pm 20,9$ & $0,0 \pm 0,0$ & $8,8 \pm 17,3$ \\
\hline Layer II /III & $1605,6 \pm 277,6$ & $22,8 \pm 43,7$ & $1591,9 \pm 287,1$ \\
\hline Layer IV & $2933 \pm 199,1$ & $62,1 \pm 68,7$ & $2870,9 \pm 246,7$ \\
\hline Layer Va & $1655,4 \pm 182,7$ & $139,7 \pm 96,9$ & $2062,2 \pm 163,1$ \\
\hline Layer Vb & $2627,5 \pm 369,8$ & $565,4 \pm 265,8$ & $3577,9 \pm 218,6$ \\
\hline Layer V (a+b) & $4282,9 \pm 382,8$ & $705 \pm 327,1$ & $1660,8 \pm 324,3$ \\
\hline Layer VI & $1710,1 \pm 336,0$ & $49,3 \pm 53,2$ & 2 \\
\hline
\end{tabular}

Tabellarische Darstellung der Anzahl der tdTomato-Zellen sowie die Anzahl der tdTomato-Zellen ohne und mit Gad1-Sonden-markierung innerhalb der einzelnen Schichten für $1 \mathrm{~mm}^{3}$ Barrel-Kortex. Die Werte sind in Mittelwerte \pm Standardabweichung angegeben.

Tabelle 6.6: Anzahl der tdTomato-Zellen und der Vglut1-positiven Zellen in den einzelnen Schichten des Barrel-Kortex

\begin{tabular}{|c|c|c|c|}
\hline Region & alle tdTomato & tdTomato & Kolok mit Vglut1 \\
\hline Barrel-Kortex & $9628,7 \pm 648,5$ & $9119,8 \pm 592,9$ & $508,9 \pm 162,1$ \\
\hline Layer I & $26,4 \pm 19,8$ & $26,4 \pm 19,8$ & $0,0 \pm 0,0$ \\
\hline Layer II/III & $1715,3 \pm 123,3$ & $1711,7 \pm 128,5$ & $3,6 \pm 10,3$ \\
\hline Layer IV & $2811,9 \pm 286$ & $2811,9 \pm 286$ & $0,0 \pm 0,0$ \\
\hline Layer Va & $1379,5 \pm 197,2$ & $1296,9 \pm 206,5$ & $82,6 \pm 65,8$ \\
\hline Layer Vb & $2211,3 \pm 340,1$ & $1796,6 \pm 233,9$ & $414,7 \pm 185,3$ \\
\hline Layer V (a+b) & $3590,8 \pm 352,7$ & $3093,5 \pm 324,8$ & $497,3 \pm 166,2$ \\
\hline Layer VI & $1478,3 \pm 255,7$ & $1470,4 \pm 257,3$ & $7,9 \pm 15,5$ \\
\hline
\end{tabular}

Tabellarische Darstellung der Anzahl der tdTomato-Zellen sowie die Anzahl der tdTomato-Zellen ohne und mit Vglut1-Sonden-markierung innerhalb der einzelnen Schichten für $1 \mathrm{~mm}^{3}$ Barrel-Kortex. Die Werte sind in Mittelwerte \pm Standardabweichung angegeben. 
Tabelle 6.7: Schichtspezifische Verteilung der kolokalisierten tdTomato-Zellen mit Gad1- und Vglut1-Sonde

\begin{tabular}{|c|c|c|c|c|}
\hline & tdTomato und Gad1-Sonde & $\begin{array}{c}\text { tdTomato und } \\
\text { Vglut1-Sonde }\end{array}$ & $\begin{array}{c}\text { Nur tdTomato- } \\
\text { Zellen } \\
\text { Verteilung in \% }\end{array}$ & $\begin{array}{c}\text { Vglut1 } \\
\text { Verteilung in } \\
\%\end{array}$ \\
\hline Region & Kolokalisation & Kolokalisation & Kolokalisation & Kolokalisation \\
\hline Barrel-Kortex & $92,1 \pm 3,3$ & $5,3 \pm 1,6$ & $100,0 \pm 40,8$ & $100,0 \pm 31,8$ \\
\hline Layer I & $100,0 \pm 0,0$ & $0,0 \pm 0,0$ & $0,0 \pm 0,0$ & $0,0 \pm 0,0$ \\
\hline Layer II/III & $98,5 \pm 3,2$ & $0,2 \pm 0,6$ & $2,7 \pm 5,2$ & $0,7 \pm 2,0$ \\
\hline Layer IV & $97,8 \pm 2,5$ & $0 \pm 0$ & $7,4 \pm 8,2$ & $0,0 \pm 0,0$ \\
\hline Layer Va & $91,6 \pm 5,7$ & $6,1 \pm 4,7$ & $16,6 \pm 11,5$ & $16,2 \pm 12,9$ \\
\hline Layer Vb & $79,3 \pm 7,9$ & $18,2 \pm 6,6$ & $67,4 \pm 31,7$ & $81,5 \pm 36,4$ \\
\hline Layer V (a+b) & $84,0 \pm 6,8$ & $13,8 \pm 4,3$ & $84,0 \pm 39,0$ & $97,7 \pm 32,7$ \\
\hline Layer VI & $97,2 \pm 2,6$ & $0,6 \pm 1,2$ & $5,9 \pm 6,3$ & $1,6 \pm 3,1$ \\
\hline
\end{tabular}

Tabellarische Darstellung der mit der Gad1- oder Vglut1-Sonde kolokalisierten tdTomato-Zellen (erste und zweite Spalte) bzw. das Vorkommen der tdTomato-Zellen aus der Gad1-Auswertung ohne Markierung (dritte Spalte) und der Vglut1-markierten Zellen (vierte Spalte). Die prozentualen Werte sind jeweils für $1 \mathrm{~mm}^{3}$ Barrel-Kortex und für die einzelnen Schichten in Mittelwerte \pm Standardabweichung angegeben. 
Tabelle 6.8: Anzahl der tdTomato-Zellen und der VIP- sowie SOM-positiven Zellen in den einzelnen Schichten des Barrel-Kortex

\begin{tabular}{|c|c|c|c|c|c|c|}
\hline Region & $\begin{array}{c}\text { alle tdTomato- } \\
\text { Zellen der } \\
\text { VIP-Schnitte }\end{array}$ & $\begin{array}{c}\text { VIP-Zellen } \\
\text { der Vip- } \\
\text { Sonde }\end{array}$ & $\begin{array}{c}\text { VIP } \\
\text { Verteilung } \\
\text { in \% }\end{array}$ & $\begin{array}{c}\text { alle } \\
\text { tdTomato- } \\
\text { Zellen der } \\
\text { SOM-Schnitte }\end{array}$ & $\begin{array}{c}\text { SOM-Zellen } \\
\text { der Sst- } \\
\text { Sonde }\end{array}$ & $\begin{array}{c}\text { SOM } \\
\text { Verteilung } \\
\text { in \% }\end{array}$ \\
\hline $\begin{array}{c}\text { Barrel- } \\
\text { Kortex }\end{array}$ & $10264,6 \pm 834,5$ & $2927,7 \pm 580,0$ & $100,0 \pm 19,8$ & $10525,5 \pm 728,2$ & $6730,8 \pm 741,6$ & $100,0 \pm 11,0$ \\
\hline Layer I & $15,8 \pm 26,5$ & $47,7 \pm 34,6$ & $1,6 \pm 1,2$ & $6,4 \pm 18,0$ & $32,6 \pm 52,7$ & $0,5 \pm 0,8$ \\
\hline $\begin{array}{c}\text { Layer } \\
\text { II/III }\end{array}$ & $1651,8 \pm 133,8$ & $1770,2 \pm 241,2$ & $60,5 \pm 8,2$ & $1519,4 \pm 233,8$ & $759,2 \pm 117,5$ & $11,3 \pm 1,7$ \\
\hline \begin{tabular}{c} 
Layer IV \\
\hline Layer Va
\end{tabular} & $1518,9 \pm 238,0$ & $86,9 \pm 60,6$ & $3,0 \pm 2,1$ & $1503,5 \pm 174,0$ & $1033,6 \pm 207,9$ & $15,4 \pm 3,1$ \\
\hline $\begin{array}{c}\text { Layer } \\
\text { Vb }\end{array}$ & $2384,3 \pm 444,0$ & $222,4 \pm 135,4$ & $7,6 \pm 4,6$ & $2700,4 \pm 509,3$ & $2214,2 \pm 402,8$ & $32,9 \pm 6,0$ \\
\hline $\begin{array}{c}\text { Layer VI } \\
1690,6 \pm 242,7\end{array}$ & $287,1 \pm 103,7$ & $9,8 \pm 3,5$ & $1785,4 \pm 164,5$ & $1431,0 \pm 651,8$ & $21,3 \pm 9,7$ \\
\hline
\end{tabular}

Tabellarische Darstellung der Anzahl der tdTomato-Zellen sowie die Anzahl der tdTomato-Zellen mit $V i p$-bzw. Sst-Sonden-markierung innerhalb der einzelnen Schichten für $1 \mathrm{~mm}^{3}$ Barrel-Kortex. Die erste, zweite, vierte und fünfte Spalte (von links) geben die Mittelwerte \pm Standardabweichung an, die dritte und sechste Spalte ihr prozentuales Vorkommen. 
Tabelle 6.9: Anzahl an kolokalisierten tdTomato-Zellen mit der Vip- und Sst-Sonde

\begin{tabular}{|c|c|c|c|c|}
\hline Region & nur VIP & Kolok & nur SOM & Kolok \\
\hline Barrel-Kortex & $2906,9 \pm 587,4$ & $20,8 \pm 27,0$ & $6171,2 \pm 938,3$ & $559,5 \pm 245,2$ \\
\hline Layer I & $47,7 \pm 34,6$ & $0,0 \pm 0,0$ & $32,6 \pm 52,7$ & $0,0 \pm 0,0$ \\
\hline Layer II/III & $1749,4 \pm 252,6$ & $20,8 \pm 27,0$ & $701,9 \pm 101,6$ & $57,3 \pm 47,4$ \\
\hline Layer IV & $520,3 \pm 207,0$ & $0,0 \pm 0,0$ & $870,9 \pm 160,9$ & $115,6 \pm 41,8$ \\
\hline Layer Va & $86,9 \pm 60,6$ & $0,0 \pm 0,0$ & $962,7 \pm 199,0$ & $70,9 \pm 14,9$ \\
\hline Layer Vb & $222,4 \pm 135,4$ & $0,0 \pm 0,0$ & $2047,5 \pm 420,0$ & $166,7 \pm 97,2$ \\
\hline Layer V (a+b) & $309,3 \pm 157,6$ & $0,0 \pm 0,0$ & $3010,2 \pm 545,8$ & $237,6 \pm 99,2$ \\
\hline Layer VI & $287,1 \pm 103,7$ & $0,0 \pm 0,0$ & $1549,6 \pm 418,3$ & $142,2 \pm 130,6$ \\
\hline
\end{tabular}

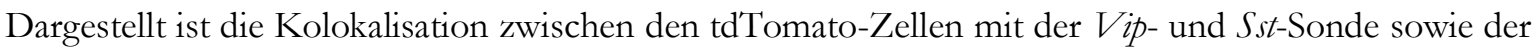
Anteil an Zellen ohne Markierung innerhalb der einzelnen Schichten für $1 \mathrm{~mm}^{3}$ Barrel-Kortex. Die Werte sind in Mittelwerte \pm Standardabweichung angegeben. 
Tabelle 6.10: Schichtspezifische Verteilung der kolokalisierten tdTomato-Zellen mit der Vipoder Sst-Sonde

\begin{tabular}{|c|c|c|c|c|}
\hline & $\begin{array}{c}\text { tdTomato und } \\
\text { Vip-Sonde }\end{array}$ & $\begin{array}{c}\text { Vip-Sonde und } \\
\text { tdTomato }\end{array}$ & $\begin{array}{c}\text { tdTomato und } \\
\text { Sst } \text {-Sonde }\end{array}$ & $\begin{array}{c}\text { Sst-Sonde und } \\
\text { tdTomato }\end{array}$ \\
\hline Region & Kolokalisation & Kolokalisation & Kolokalisation & Kolokalisation \\
\hline Barrel-Kortex & $0,2 \pm 0,2$ & $0,8 \pm 1,2$ & $5,3 \pm 2,4$ & $8,7 \pm 4,6$ \\
\hline Layer I & $0,0 \pm 0,0$ & $0,0 \pm 0,0$ & $0,0 \pm 0,0$ & $0,0 \pm 0,0$ \\
\hline Layer II/III & $1,3 \pm 1,7$ & $1,3 \pm 1,8$ & $3,6 \pm 3,0$ & $7,3 \pm 5,5$ \\
\hline Layer IV & $0,0 \pm 0,0$ & $0,0 \pm 0,0$ & $3,8 \pm 1,3$ & $12,0 \pm 5,0$ \\
\hline Layer Va & $0,0 \pm 0,0$ & $0,0 \pm 0,0$ & $4,8 \pm 1,0$ & $7,0 \pm 1,4$ \\
\hline Layer Vb & $0,0 \pm 0,0$ & $0,0 \pm 0,0$ & $6,0 \pm 3,2$ & $7,7 \pm 4,5$ \\
\hline Layer VI & $0,0 \pm 0,0$ & $0,0 \pm 0,0$ & $8,0 \pm 7,0$ & $9,0 \pm 9,0$ \\
\hline
\end{tabular}

Tabellarische Darstellung der mit der Vip- oder Sst-Sonde kolokalisierten tdTomato-Zellen bzw. der VIP- oder SOM-positiven Zellen mit dem tdTomato-Signal. Die prozentualen Werte sind jeweils für 1 $\mathrm{mm}^{3}$ Barrel-Kortex und für die einzelnen Schichten in Mittelwerte \pm Standardabweichung angegeben. Die erste Spalte (von links) zeigt wie viel Prozent der tdTomato-Zellen, die mit der Vip-Sonde markiert sind. Die zweite Spalte stellt umgekehrt die markierten Zellen mit Vip-Sonde, die zusätzlich die tdTomato-Fluoreszenz zeigen, dar. Analog sind in der Spalte drei und vier die Daten für die Sst-Sonde angegeben.

Tabelle 6.11: Anzahl der Zellen in der WFA-Färbung in den einzelnen Schichten des BarrelKortex

\begin{tabular}{|c|c|c|c|c|c|}
\hline Region & alle tdTomato & tdTomato & alle WFA & WFA & Kolok \\
\hline Barrel-Kortex & $9748,5 \pm 513,2$ & $2839,0 \pm 603,4$ & $6964,3 \pm 575,1$ & $54,8 \pm 58,7$ & $6909,6 \pm 587,3$ \\
\hline Layer I & $2,9 \pm 8,3$ & $2,9 \pm 8,3$ & $0,0 \pm 0,0$ & $0,0 \pm 0,0$ & $0,0 \pm 0,0$ \\
\hline Layer II/III & $1621,4 \pm 216,0$ & $562,0 \pm 194,1$ & $1062,9 \pm 91,7$ & $3,5 \pm 9,9$ & $1059,4 \pm 95,8$ \\
\hline Layer IV & $2757,9 \pm 130,0$ & $219,9 \pm 112,1$ & $2553,5 \pm 146,3$ & $15,5 \pm 25,5$ & $2538,0 \pm 136,1$ \\
\hline Layer Va & $1552,3 \pm 270,1$ & $485,7 \pm 275,4$ & $1072,9 \pm 185,7$ & $6,4 \pm 11,8$ & $1066,6 \pm 179,6$ \\
\hline Layer Vb & $2561,4 \pm 321,3$ & $1137,1 \pm 230,8$ & $1443,4 \pm 200,3$ & $19,1 \pm 22,9$ & $1424,3 \pm 209,8$ \\
\hline Layer V (a+b) & $4113,6 \pm 402,3$ & $1622,8 \pm 365,1$ & $2516,3 \pm 343,3$ & $25,5 \pm 24,1$ & $2490,8 \pm 343,4$ \\
\hline Layer VI & $1240,2 \pm 226,6$ & $431,4 \pm 109,2$ & $819,1 \pm 228,6$ & $10,3 \pm 20,6$ & $808,8 \pm 224,9$ \\
\hline
\end{tabular}

Tabellarische Darstellung der Anzahl der kolokalisierten und nicht-kolokalisierten tdTomato-Zellen mit dem WFA-Signal, sowie der WFA-positiven Zellen innerhalb der einzelnen Schichten für $1 \mathrm{~mm}^{3}$ BarrelKortex. Die Werte sind in Mittelwerte \pm Standardabweichung angegeben. 
Tabelle 6.12: Prozentuale schichtspezifische Verteilung der Zellen, die nur tdTomato-positiv oder nur WFA-positiv sind sowie die kolokalisierten Zellen

\begin{tabular}{|c|c|c|c|}
\hline Region & nur tdTomato & nur WFA & Kolok \\
\hline Barrel-Kortex & $100,0 \pm 21,3$ & $100,0 \pm 107,2$ & $100,0 \pm 8,5$ \\
\hline Layer I & $0,1 \pm 0,3$ & $0,0 \pm 0,0$ & $0,0 \pm 0,0$ \\
\hline Layer II/III & $19,8 \pm 6,8$ & $6,4 \pm 18,1$ & $15,3 \pm 1,4$ \\
\hline Layer IV & $7,7 \pm 3,9$ & $28,3 \pm 46,6$ & $36,7 \pm 2,0$ \\
\hline Layer Va & $17,1 \pm 9,7$ & $11,6 \pm 21,6$ & $15,4 \pm 2,6$ \\
\hline Layer Vb & $40,1 \pm 8,1$ & $34,9 \pm 41,8$ & $20,6 \pm 3,0$ \\
\hline Layer V (a+b) & $57,2 \pm 12,9$ & $46,5 \pm 44,1$ & $36,0 \pm 5,0$ \\
\hline Layer VI & $15,2 \pm 3,8$ & $18,8 \pm 37,7$ & $11,7 \pm 3,3$ \\
\hline
\end{tabular}

Tabellarische Darstellung der prozentualen Verteilung von nur tdTomato-Zellen, nur WFA-positiver und deren Kolokalisation innerhalb der einzelnen Schichten für $1 \mathrm{~mm}^{3}$ Barrel-Kortex. Die Werte sind in Mittelwerte \pm Standardabweichung angegeben.

Tabelle 6.13: Schichtspezifische Verteilung der kolokalisierten tdTomato-Zellen mit dem WFASignal

\begin{tabular}{|c|c|c|}
\hline Region & tdTomato mit WFA kolokalisiert & WFA mit tdTomato kolokalisiert \\
\hline Barrel-Kortex & $70,9 \pm 5,6$ & $99,2 \pm 0,9$ \\
\hline Layer I & $0,0 \pm 0,0$ & keine WFA-Markierung \\
\hline Layer II /III & $66,1 \pm 8,3$ & $99,64 \pm 1,01$ \\
\hline Layer IV & $92,1 \pm 3,9$ & $99,4 \pm 0,9$ \\
\hline Layer Va & $70,0 \pm 14,4$ & $99,5 \pm 1,0$ \\
\hline Layer Vb & $55,7 \pm 6,0$ & $98,6 \pm 1,7$ \\
\hline Layer V (a+b) & $60,7 \pm 7,1$ & $99,0 \pm 7,1$ \\
\hline Layer VI & $64,6 \pm 9,0$ & $98,5 \pm 2,7$ \\
\hline
\end{tabular}

Tabellarische Darstellung der mit dem WFA-Signal kolokalisierten tdTomato-Zellen bzw. der WFApositiven Zellen mit dem tdTomato-Signal. Die prozentualen Werte sind jeweils für $1 \mathrm{~mm}^{3}$ BarrelKortex und für die einzelnen Schichten in Mittelwerte \pm Standardabweichung angegeben. Die erste Spalte (von links) zeigt wie viel Prozent der tdTomato-Zellen, auch für WFA-positiv sind. Die zweite Spalte stellt umgekehrt die markierten Zellen mit dem WFA-Signal, die zusätzlich die tdTomatoFluoreszenz zeigen, dar. 
Anhang

Tabelle 6.14: Ergebnisse des Mann-Whitney-Ranksummentests

\begin{tabular}{|c|c|c|c|c|c|}
\hline \multicolumn{7}{|c|}{ Statistik IHC PV-markierten tdTomato-Schnitte } \\
\hline & LayerII/III & LayerIV & LayerVa & LayerVb & LayerVI \\
\hline LayerII/III & -- & $(\mathrm{P}=<0,001)$ & $(\mathrm{P}=0,038)$ & $(\mathrm{P}=<0,001)$ & $(\mathrm{P}=0,038)$ \\
\hline LayerIV & $(\mathrm{P}=<0,001)$ & -- & $(\mathrm{P}=<0,001)$ & $(\mathrm{P}=0,053)$ & $(\mathrm{P}=<0,001)$ \\
\hline LayerVa & $(\mathrm{P}=0,038)$ & $(\mathrm{P}=<0,001)$ & -- & $(\mathrm{P}=<0,001)$ & $(\mathrm{P}=0,128)$ \\
\hline LayerVb & $(\mathrm{P}=<0,001)$ & $(\mathrm{P}=0,053)$ & $(\mathrm{P}=<0,001)$ & -- & $(\mathrm{P}=<0,001)$ \\
\hline LayerVI & $(\mathrm{P}=0,038)$ & $(\mathrm{P}=<0,001)$ & $(\mathrm{P}=0,128)$ & $(\mathrm{P}=<0,001)$ & -- \\
\hline
\end{tabular}

\begin{tabular}{|c|c|c|c|c|c|}
\hline \multicolumn{7}{|c|}{ Statistik der relativen Werte IHC PV-markierten tdTomato-Schnitte } \\
\hline & LayerII/III & LayerIV & LayerVa & LayerVb & LayerVI \\
\hline LayerII/III & -- & $(\mathrm{P}=1,000)$ & $(\mathrm{P}=0,318)$ & $(\mathrm{P}=0,038)$ & $(\mathrm{P}=1,000)$ \\
\hline LayerIV & $(\mathrm{P}=1,000)$ & -- & $(\mathrm{P}=0,535)$ & $(\mathrm{P}=0,073)$ & $(\mathrm{P}=0,535)$ \\
\hline LayerVa & $(\mathrm{P}=0,318)$ & $(\mathrm{P}=0,535)$ & -- & $(\mathrm{P}=0,073)$ & $(\mathrm{P}=0,456)$ \\
\hline LayerVb & $(\mathrm{P}=0,038)$ & $(\mathrm{P}=0,073)$ & $(\mathrm{P}=0,073)$ & -- & $(\mathrm{P}=0,165)$ \\
\hline LayerVI & $(\mathrm{P}=1,000)$ & $(\mathrm{P}=0,535)$ & $(\mathrm{P}=0,456)$ & $(\mathrm{P}=0,165)$ & -- \\
\hline
\end{tabular}

\begin{tabular}{|c|c|c|c|c|c|}
\hline \multicolumn{7}{|c|}{ Statistik FISH Gad1-markierten tdTomato-Zellen } \\
\hline & LayerII/III & LayerIV & LayerVa & LayerVb & LayerVI \\
\hline LayerII/III & -- & $(\mathrm{P}=<0,001)$ & $(\mathrm{P}=0,574)$ & $(\mathrm{P}=0,005)$ & $(\mathrm{P}=0,328)$ \\
\hline LayerIV & $(\mathrm{P}=<0,001)$ & -- & $(\mathrm{P}=<0,001)$ & $(\mathrm{P}=<0,001)$ & $(\mathrm{P}=<0,001)$ \\
\hline LayerVa & $(\mathrm{P}=0,574)$ & $(\mathrm{P}=<0,001)$ & -- & $(\mathrm{P}=<0,001)$ & $(\mathrm{P}=0,130)$ \\
\hline LayerVb & $(\mathrm{P}=0,005)$ & $(\mathrm{P}=<0,001)$ & $(\mathrm{P}=<0,001)$ & -- & $(\mathrm{P}=0,003)$ \\
\hline LayerVI & $(\mathrm{P}=0,328)$ & $(\mathrm{P}=<0,001)$ & $(\mathrm{P}=0,130)$ & $(\mathrm{P}=0,003)$ & -- \\
\hline
\end{tabular}

\begin{tabular}{|c|c|c|c|c|c|}
\hline \multicolumn{7}{|c|}{ Statistik der relativen Werte FISH Gad1-markierten tdTomato-Zellen } \\
\hline & LayerII/III & LayerIV & LayerVa & LayerVb & LayerVI \\
\hline LayerII/III & -- & $(\mathrm{P}=0,382)$ & $(\mathrm{P}=0,003)$ & $(\mathrm{P}=<0,001)$ & $(\mathrm{P}=0,083)$ \\
\hline LayerIV & $(\mathrm{P}=0,382)$ & -- & $(\mathrm{P}=0,021)$ & $(\mathrm{P}=<0,001)$ & $(\mathrm{P}=0,645)$ \\
\hline LayerVa & $(\mathrm{P}=0,003)$ & $(\mathrm{P}=0,021)$ & -- & $(\mathrm{P}=0,003)$ & $(\mathrm{P}=0,050)$ \\
\hline LayerVb & $(\mathrm{P}=<0,001)$ & $(\mathrm{P}=<0,001)$ & $(\mathrm{P}=0,003)$ & -- & $(\mathrm{P}=<0,001)$ \\
\hline LayerVI & $(\mathrm{P}=0,083)$ & $(\mathrm{P}=0,645)$ & $(\mathrm{P}=0,050)$ & $(\mathrm{P}=<0,001)$ & -- \\
\hline
\end{tabular}

\begin{tabular}{|c|c|c|c|c|c|}
\hline \multicolumn{7}{|c|}{ Statistik FISH Vlut1-markierten tdTomato-Zellen } \\
\hline & LayerII/III & LayerIV & LayerVa & LayerVb & LayerVI \\
\hline LayerII/III & -- & $(\mathrm{P}=0,721)$ & $(\mathrm{P}=0,005)$ & $(\mathrm{P}=<0,001)$ & $(\mathrm{P}=0,721)$ \\
\hline LayerIV & $(\mathrm{P}=0,721)$ & -- & $(\mathrm{P}=0,002)$ & $(\mathrm{P}=<0,001)$ & $(\mathrm{P}=0,442)$ \\
\hline LayerVa & $(\mathrm{P}=0,005)$ & $(\mathrm{P}=0,002)$ & -- & $(\mathrm{P}=0,001)$ & $(\mathrm{P}=0,010)$ \\
\hline LayerVb & $(\mathrm{P}=<0,001)$ & $(\mathrm{P}=<0,001)$ & $(\mathrm{P}=0,001)$ & -- & $(\mathrm{P}=0,001)$ \\
\hline LayerVI & $(\mathrm{P}=0,721)$ & $(\mathrm{P}=0,442)$ & $(\mathrm{P}=0,010)$ & $(\mathrm{P}=0,001)$ & -- \\
\hline
\end{tabular}




\begin{tabular}{|c|c|c|c|c|c|}
\hline \multicolumn{7}{|c|}{ Statistik der relativen Werte FISH Vlut1-markierten tdTomato-Zellen } \\
\hline & LayerII/III & LayerIV & LayerVa & LayerVb & LayerVI \\
\hline LayerII/III & -- & $(\mathrm{P}=0,721)$ & $(\mathrm{P}=0,005)$ & $(\mathrm{P}=<0,001)$ & $(\mathrm{P}=0,721)$ \\
\hline LayerIV & $(\mathrm{P}=0,721)$ & -- & $(\mathrm{P}=0,002)$ & $(\mathrm{P}=<0,001)$ & $(\mathrm{P}=0,442)$ \\
\hline LayerVa & $(\mathrm{P}=0,005)$ & $(\mathrm{P}=0,002)$ & -- & $(\mathrm{P}=0,002)$ & $(\mathrm{P}=0,007)$ \\
\hline LayerVb & $(\mathrm{P}=<0,001)$ & $(\mathrm{P}=<0,001)$ & $(\mathrm{P}=0,002)$ & -- & $(\mathrm{P}=<0,001)$ \\
\hline LayerVI & $(\mathrm{P}=0,721)$ & $(\mathrm{P}=0,442)$ & $(\mathrm{P}=0,007)$ & $(\mathrm{P}=<0,001)$ & -- \\
\hline
\end{tabular}

\begin{tabular}{|c|c|c|c|c|c|}
\hline \multicolumn{7}{|c|}{ Statistik FISH VIP-markierten tdTomato-Zellen } \\
\hline & LayerII/III & LayerIV & LayerVa & LayerVb & LayerVI \\
\hline LayerII/III & -- & $(\mathrm{P}=0,105)$ & $(\mathrm{P}=0,105)$ & $(\mathrm{P}=0,105)$ & $(\mathrm{P}=0,105)$ \\
\hline LayerIV & $(\mathrm{P}=0,105)$ & -- & $(\mathrm{P}=1,000)$ & $(\mathrm{P}=1,000)$ & $(\mathrm{P}=1,000)$ \\
\hline LayerVa & $(\mathrm{P}=0,105)$ & $(\mathrm{P}=1,000)$ & -- & $(\mathrm{P}=1,000)$ & $(\mathrm{P}=1,000)$ \\
\hline LayerVb & $(\mathrm{P}=0,105)$ & $(\mathrm{P}=1,000)$ & $(\mathrm{P}=1,000)$ & -- & $(\mathrm{P}=1,000)$ \\
\hline LayerVI & $(\mathrm{P}=0,105)$ & $(\mathrm{P}=1,000)$ & $(\mathrm{P}=1,000)$ & $(\mathrm{P}=1,000)$ & -- \\
\hline
\end{tabular}

\begin{tabular}{|c|c|c|c|c|c|}
\hline \multicolumn{7}{|c|}{ Statistik der relativen Werte FISH VIP-markierten Zellen } \\
\hline & LayerII/III & LayerIV & LayerVa & LayerVb & LayerVI \\
\hline LayerII/III & -- & $(\mathrm{P}=0,105)$ & $(\mathrm{P}=0,105)$ & $(\mathrm{P}=0,105)$ & $(\mathrm{P}=0,105)$ \\
\hline LayerIV & $(\mathrm{P}=0,105)$ & -- & $(\mathrm{P}=1,000)$ & $(\mathrm{P}=1,000)$ & $(\mathrm{P}=1,000)$ \\
\hline LayerVa & $(\mathrm{P}=0,105)$ & $(\mathrm{P}=1,000)$ & -- & $(\mathrm{P}=1,000)$ & $(\mathrm{P}=1,000)$ \\
\hline LayerVb & $(\mathrm{P}=0,105)$ & $(\mathrm{P}=1,000)$ & $(\mathrm{P}=1,000)$ & -- & $(\mathrm{P}=1,000)$ \\
\hline LayerVI & $(\mathrm{P}=0,105)$ & $(\mathrm{P}=1,000)$ & $(\mathrm{P}=1,000)$ & $(\mathrm{P}=1,000)$ & -- \\
\hline
\end{tabular}

\begin{tabular}{|c|c|c|c|c|c|}
\hline \multicolumn{7}{|c|}{ Statistik FISH SOM-markierten tdTomato-Zellen } \\
\hline & LayerII/III & LayerIV & LayerVa & LayerVb & LayerVI \\
\hline LayerII/III & -- & $(\mathrm{P}=0,021)$ & $(\mathrm{P}=0,505)$ & $(\mathrm{P}=0,015)$ & $(\mathrm{P}=0,105)$ \\
\hline LayerIV & $(\mathrm{P}=0,021)$ & -- & $(\mathrm{P}=0,021)$ & $(\mathrm{P}=0,328)$ & $(\mathrm{P}=0,798)$ \\
\hline LayerVa & $(\mathrm{P}=0,505)$ & $(\mathrm{P}=0,021)$ & -- & $(\mathrm{P}=0,065)$ & $(\mathrm{P}=0,195)$ \\
\hline LayerVb & $(\mathrm{P}=0,015)$ & $(\mathrm{P}=0,328)$ & $(\mathrm{P}=0,065)$ & -- & $(\mathrm{P}=0,505)$ \\
\hline LayerVI & $(\mathrm{P}=0,105)$ & $(\mathrm{P}=0,798)$ & $(\mathrm{P}=0,195)$ & $(\mathrm{P}=0,505)$ & -- \\
\hline
\end{tabular}

\begin{tabular}{|c|c|c|c|c|c|}
\hline \multicolumn{7}{|c|}{ Statistik der relativen Werte FISH SOM-markierten tdTomato-Zellen } \\
\hline & LayerII/III & LayerIV & LayerVa & LayerVb & LayerVI \\
\hline LayerII/III & -- & $(\mathrm{P}=0,798)$ & $(\mathrm{P}=0,279)$ & $(\mathrm{P}=0,130)$ & $(\mathrm{P}=0,105)$ \\
\hline LayerIV & $(\mathrm{P}=0,798)$ & -- & $(\mathrm{P}=0,279)$ & $(\mathrm{P}=0,083)$ & $(\mathrm{P}=0,234)$ \\
\hline LayerVa & $(\mathrm{P}=0,279)$ & $(\mathrm{P}=0,279)$ & -- & $(\mathrm{P}=0,279)$ & $(\mathrm{P}=0,645)$ \\
\hline LayerVb & $(\mathrm{P}=0,130)$ & $(\mathrm{P}=0,083)$ & $(\mathrm{P}=0,279)$ & -- & $(\mathrm{P}=0,878)$ \\
\hline LayerVI & $(\mathrm{P}=0,105)$ & $(\mathrm{P}=0,234)$ & $(\mathrm{P}=0,645)$ & $(\mathrm{P}=0,878)$ & -- \\
\hline
\end{tabular}


Statistik IHC WFA-markierten tdTomato-Zellen

\begin{tabular}{|c|c|c|c|c|c|}
\hline & LayerII/III & LayerIV & LayerVa & LayerVb & LayerVI \\
\hline LayerII/III & -- & $(\mathrm{P}=<0,001)$ & $(\mathrm{P}=1,000)$ & $(\mathrm{P}=<0,001)$ & $(\mathrm{P}=0,021)$ \\
\hline LayerIV & $(\mathrm{P}=<0,001)$ & -- & $(\mathrm{P}=<0,001)$ & $(\mathrm{P}=<0,001)$ & $(\mathrm{P}=<0,001)$ \\
\hline LayerVa & $(\mathrm{P}=1,000)$ & $(\mathrm{P}=<0,001)$ & -- & $(\mathrm{P}=0,002)$ & $(\mathrm{P}=0,028)$ \\
\hline LayerVb & $(\mathrm{P}=<0,001)$ & $(\mathrm{P}=<0,001)$ & $(\mathrm{P}=0,002)$ & -- & $(\mathrm{P}=<0,001)$ \\
\hline LayerVI & $(\mathrm{P}=0,021)$ & $(\mathrm{P}=<0,001)$ & $(\mathrm{P}=0,028)$ & $(\mathrm{P}=<0,001)$ & -- \\
\hline
\end{tabular}

\begin{tabular}{|c|c|c|c|c|c|}
\hline \multicolumn{7}{|c|}{ Statistik der relativen Werte IHC WFA-markierten tdTomato-Zellen } \\
\hline & LayerII/III & LayerIV & LayerVa & LayerVb & LayerVI \\
\hline LayerII/III & -- & $(\mathrm{P}=<0,001)$ & $(\mathrm{P}=0,645)$ & $(\mathrm{P}=0,021)$ & $(\mathrm{P}=0,574)$ \\
\hline LayerIV & $(\mathrm{P}=<0,001)$ & -- & $(\mathrm{P}=0,010)$ & $(\mathrm{P}=<0,001)$ & $(\mathrm{P}=<0,001)$ \\
\hline LayerVa & $(\mathrm{P}=0,645)$ & $(\mathrm{P}=0,010)$ & -- & $(\mathrm{P}=0,038)$ & $(\mathrm{P}=0,574)$ \\
\hline LayerVb & $(\mathrm{P}=0,021)$ & $(\mathrm{P}=<0,001)$ & $(\mathrm{P}=0,038)$ & -- & $(\mathrm{P}=0,028)$ \\
\hline LayerVI & $(\mathrm{P}=0,574)$ & $(\mathrm{P}=<0,001)$ & $(\mathrm{P}=0,574)$ & $(\mathrm{P}=0,028)$ & -- \\
\hline
\end{tabular}

\begin{tabular}{|c|c|c|c|c|c|}
\hline \multicolumn{7}{|c|}{ Statistik aller PV-markierten tdTomato-Zellen } \\
\hline & LayerII/III & LayerIV & LayerVa & LayerVb & LayerVI \\
\hline LayerII/III & -- & $(\mathrm{P}=<0,001)$ & $(\mathrm{P}=0,012)$ & $(\mathrm{P}=<0,001)$ & $(\mathrm{P}=0,366)$ \\
\hline LayerIV & $(\mathrm{P}=<0,001)$ & -- & $(\mathrm{P}=<0,001)$ & $(\mathrm{P}=<0,001)$ & $(\mathrm{P}=<0,001)$ \\
\hline LayerVa & $(\mathrm{P}=0,012)$ & $(\mathrm{P}=<0,001)$ & -- & $(\mathrm{P}=<0,001)$ & $(\mathrm{P}=0,427)$ \\
\hline LayerVb & $(\mathrm{P}=<0,001)$ & $(\mathrm{P}=<0,001)$ & $(\mathrm{P}=<0,001)$ & -- & $(\mathrm{P}=<0,001)$ \\
\hline LayerVI & $(\mathrm{P}=0,366)$ & $(\mathrm{P}=<0,001)$ & $(\mathrm{P}=0,427)$ & $(\mathrm{P}=<0,001)$ & -- \\
\hline
\end{tabular}

\begin{tabular}{|c|c|c|c|c|c|}
\hline \multicolumn{7}{|c|}{ Statistik der relativen Werte aller PV-markierten tdTomato-Zellen } \\
\hline & LayerII/III & LayerIV & LayerVa & LayerVb & LayerVI \\
\hline LayerII/III & -- & $(\mathrm{P}=<0,001)$ & $(\mathrm{P}=0,008)$ & $(\mathrm{P}=<0,001)$ & $(\mathrm{P}=0,093)$ \\
\hline LayerIV & $(\mathrm{P}=<0,001)$ & -- & $(\mathrm{P}=<0,001)$ & $(\mathrm{P}=<0,001)$ & $(\mathrm{P}=<0,001)$ \\
\hline LayerVa & $(\mathrm{P}=0,008)$ & $(\mathrm{P}=<0,001)$ & -- & $(\mathrm{P}=<0,001)$ & $(\mathrm{P}=0,463)$ \\
\hline LayerVb & $(\mathrm{P}=<0,001)$ & $(\mathrm{P}=<0,001)$ & $(\mathrm{P}=<0,001)$ & -- & $(\mathrm{P}=<0,001)$ \\
\hline LayerVI & $(\mathrm{P}=0,093)$ & $(\mathrm{P}=<0,001)$ & $(\mathrm{P}=0,463)$ & $(\mathrm{P}=<0,001)$ & -- \\
\hline
\end{tabular}

Dargestellt sind die tabellarischen Ergebnisse des Mann-Whitney-Ranksummentest für die absoluten Werte (links) und die relativen Werte (rechts). Verglichen wurden dabei die Anzahl der kolokalisierten tdTomato-Zellen (absolut) bzw. die prozentuale Kolokalisationsquote (relativ) mit dem angegebenen Marker zwischen den einzelnen Schichten. Des Weiteren enthalten die letzten beiden Tabellen die Ergebnisse des Mann-Whitney-Ranksummentest für die Verteilung der tdTomato-positiven Zellen in der PVcre-Maus aller 47 untersuchten Hirnschnitte. Signifikante Werte $(p<0,05)$ sind rot dargestellt. Die Schicht I wurde aufgrund der geringen Zellzahl nicht aufgeführt. 
Tabelle 6.15: Verwendete Sonden der FISH

\begin{tabular}{|c|c|c|c|c|c|}
\hline $\begin{array}{c}\mathrm{Ge} \\
\mathrm{n}\end{array}$ & $\begin{array}{l}\text { Lä } \\
\text { nge }\end{array}$ & Forward primer & Reverse primer & $\begin{array}{l}\text { Nested primer } \\
\quad \text { (forward) }\end{array}$ & $\begin{array}{l}\text { Nested primer } \\
\text { (reverse) }\end{array}$ \\
\hline $\begin{array}{l}G a \\
d 1\end{array}$ & $\begin{array}{l}320 \\
\text { BP }\end{array}$ & $\begin{array}{c}\text { GGCACGACTGTT } \\
\text { TACGGAGC }\end{array}$ & $\begin{array}{c}\text { GCCTTGTCCCCG } \\
\text { GTGTCATA }\end{array}$ & - & - \\
\hline Sst & $\begin{array}{l}514 \\
\text { BP }\end{array}$ & $\begin{array}{c}\text { ACGCTACCGAAG } \\
\text { CCGTC }\end{array}$ & $\begin{array}{c}\text { GGGGCCAGGAG } \\
\text { TTAAGGA }\end{array}$ & - & - \\
\hline $\begin{array}{l}V g l \\
u t 1\end{array}$ & $\begin{array}{l}296 \\
\text { BP }\end{array}$ & $\begin{array}{c}\text { CAGAGCCGGAG } \\
\text { GAGATGA }\end{array}$ & $\begin{array}{c}\text { TTCССTCAGAAAC } \\
\text { GCTGG }\end{array}$ & $\begin{array}{c}\text { GCTGGCAGTGA } \\
\text { CGAAAGTGA }\end{array}$ & $\begin{array}{c}\text { TGAGAGGGAAA } \\
\text { GAGGGCTGG }\end{array}$ \\
\hline Vip & $\begin{array}{l}367 \\
\text { BP }\end{array}$ & $\begin{array}{c}\text { СCTGGCATTCCT } \\
\text { GATACTCTTC }\end{array}$ & $\begin{array}{c}\text { ATTCTCTGATTTC } \\
\text { AGCTCTGCC }\end{array}$ & $\begin{array}{c}\text { CTGTTCTCTCAG } \\
\text { TCGCTGGC }\end{array}$ & $\begin{array}{c}\text { GCTTTCTGAGGC } \\
\text { GGGTGTAG }\end{array}$ \\
\hline
\end{tabular}

In der Tabelle befinden sich die für die FISH eingesetzten Sonden mit Längenangabe und der entsprechenden Primer-Sequenzen. Die Sequenzen der Sonden Sst, Vglut1 und Vip finden Übereinstimmung mit den Sequenzen des Allen Institute for Brain Science. 


\section{$7 \quad$ Literaturverzeichnis}

Aihara Y, Mashima H, Onda H, Hisano S, Kasuya H, Hori T, Yamada S, Tomura H, Yamada Y, Inoue I, et al. (2000): Molecular cloning of a novel brain-type $\mathrm{Na}(+)$-dependent inorganic phosphate cotransporter. J Neurochem $\underline{74}, 2622-2625$

Bayraktar T, Welker E, Freund TF, Zilles K, Staiger JF (2000): Neurons immunoreactive for vasoactive intestinal polypeptide in the rat primary somatosensory cortex: morphology and spatial relationship to barrelrelated columns. J Comp Neurol $\underline{420}$, 291-304

Bellocchio EE, Reimer RJ, Fremeau RT, Edwards RH (2000): Uptake of glutamate into synaptic vesicles by an inorganic phosphate transporter. Science 289, 957-960

Besser S, Sicker M, Marx G, Winkler U, Eulenburg V, Hülsmann S, Hirrlinger J (2015): A Transgenic Mouse Line Expressing the Red Fluorescent Protein tdTomato in GABAergic Neurons. PLOS ONE $\underline{10}$, $\mathrm{e} 0129934$

Bitanihirwe BKY, Woo T-UW (2014): Perineuronal nets and schizophrenia: the importance of neuronal coatings. Neurosci Biobehav Rev $\underline{45}, 85-99$

Blatow M, Rozov A, Katona I, Hormuzdi SG, Meyer AH, Whittington MA, Caputi A, Monyer H (2003): A novel network of multipolar bursting interneurons generates theta frequency oscillations in neocortex. Neuron $\underline{38}, 805-817$

Brodmann K: Brodmann's localisation in the cerebral cortex. Übersetzt von Gary LJ. 1. Auflage; Springer US, New York 2006

Buzsáki G, Draguhn A (2004): Neuronal oscillations in cortical networks. Science $\underline{304,1926-1929}$

Cabungcal J-H, Steullet P, Morishita H, Kraftsik R, Cuenod M, Hensch TK, Do KQ (2013): Perineuronal nets protect fast-spiking interneurons against oxidative stress. Proc Natl Acad Sci U S A 110, 9130-9135

Cardin JA, Carlén M, Meletis K, Knoblich U, Zhang F, Deisseroth K, Tsai L-H, Moore CI (2009): Driving fast-spiking cells induces gamma rhythm and controls sensory responses. Nature $\underline{459}$, 663-667

Cauli B, Audinat E, Lambolez B, Angulo MC, Ropert N, Tsuzuki K, Hestrin S, Rossier J (1997): Molecular and physiological diversity of cortical nonpyramidal cells. J Neurosci 17, 3894-3906

Chevaleyre V, Piskorowski R (2014): Modulating excitation through plasticity at inhibitory synapses. Front Cell Neurosci doi: 10.3389/fncel.2014.00093

Cruikshank SJ, Lewis TJ, Connors BW (2007): Synaptic basis for intense thalamocortical activation of feedforward inhibitory cells in neocortex. Nat Neurosci 10, 462-468

Cruikshank SJ, Urabe H, Nurmikko AV, Connors BW (2010): Pathway-specific feedforward circuits between thalamus and neocortex revealed by selective optical stimulation of axons. Neuron $\underline{65}, 230-245$

DeFelipe J, Alonso-Nanclares L, Arellano JI (2002): Microstructure of the neocortex: comparative aspects. J Neurocytol 31, 299-316

Dori I, Dinopoulos A, Cavanagh ME, Parnavelas JG (1992): Proportion of glutamate- and aspartateimmunoreactive neurons in the efferent pathways of the rat visual cortex varies according to the target. J Comp Neurol 319, 191-204

Feldmeyer D (2012): Excitatory neuronal connectivity in the barrel cortex. Front Neuroanat $\underline{6}, 24$

Feldmeyer D, Brecht M, Helmchen F, Petersen CCH, Poulet JFA, Staiger JF, Luhmann HJ, Schwarz C (2013): Barrel cortex function. Prog Neurobiol 103, 3-27

Feldmeyer D, Qi G, Emmenegger V, Staiger JF (2018): Inhibitory interneurons and their circuit motifs in the many layers of the barrel cortex. Neuroscience $\underline{368}, 132-151$ 
Férézou I, Cauli B, Hill EL, Rossier J, Hamel E, Lambolez B (2002): 5-HT3 Receptors Mediate Serotonergic Fast Synaptic Excitation of Neocortical Vasoactive Intestinal Peptide/Cholecystokinin Interneurons. J Neurosci 22, 7389-7397

Fischer T: Schichtenspezifische Charakterisierung Somatostatin-exprimierender Interneurone in der GIN- und SOMcre/tdTomato-Maus mittels neurochemischer Marker im primären somatosensorischen Barrel-Kortex. Med. Diss. Göttingen 2018

Forssmann WG, Heym C: Grundriß der Neuroanatomie; 2. Auflage; Springer-Verlag Berlin Heidelberg 2013

Fremeau RT, Troyer MD, Pahner I, Nygaard GO, Tran CH, Reimer RJ, Bellocchio EE, Fortin D, Storm-Mathisen J, Edwards RH (2001): The expression of vesicular glutamate transporters defines two classes of excitatory synapse. Neuron $\underline{31}, 247-260$

Gabernet L, Jadhav SP, Feldman DE, Carandini M, Scanziani M (2005): Somatosensory integration controlled by dynamic thalamocortical feed-forward inhibition. Neuron $\underline{48}, 315-327$

Girard F, Meszar Z, Marti C, Davis FP, Celio M (2011): Gene expression analysis in the parvalbuminimmunoreactive PV1 nucleus of the mouse lateral hypothalamus. Eur J Neurosci 34, 1934-1943

Guy J, Staiger JF (2017): The Functioning of a Cortex without Layers. Front Neuroanat doi: 10.3389 / fnana.2017.00054

Halabisky B, Shen F, Huguenard JR, Prince DA (2006): Electrophysiological classification of somatostatinpositive interneurons in mouse sensorimotor cortex. J Neurophysiol $\underline{96}$, 834-845

Herzog E, Gilchrist J, Gras C, Muzerelle A, Ravassard P, Giros B, Gaspar P, E1 Mestikawy S (2004): Localization of VGLUT3, the vesicular glutamate transporter type 3, in the rat brain. Neuroscience 123 , 9831002

Hu H, Cavendish JZ, Agmon A (2013): Not all that glitters is gold: off-target recombination in the somatostatin-IRES-Cre mouse line labels a subset of fast-spiking interneurons. Front Neural Circuits doi: 10.3389 /fncir.2013.00195

Jensen O, Kaiser J, Lachaux J-P (2007): Human gamma-frequency oscillations associated with attention and memory. Trends Neurosci $\underline{30}, 317-324$

Jiang X, Shen S, Cadwell CR, Berens P, Sinz F, Ecker AS, Patel S, Tolias AS (2015): Principles of connectivity among morphologically defined cell types in adult neocortex. Science $\underline{350}$, aac 9462

Karetko M, Skangiel-Kramska J (2009): Diverse functions of perineuronal nets. Acta Neurobiol Exp (Wars) $\underline{69}, 564-577$

Karnani MM, Jackson J, Ayzenshtat I, Hamzehei Sichani A, Manoocheri K, Kim S, Yuste R (2016): Opening Holes in the Blanket of Inhibition: Localized Lateral Disinhibition by VIP Interneurons. J Neurosci $\underline{36}$, $3471-3480$

Kaufman DL, Houser CR, Tobin AJ (1991): 'Two forms of the gamma-aminobutyric acid synthetic enzyme glutamate decarboxylase have distinct intraneuronal distributions and cofactor interactions. J Neurochem $\underline{56}$, $720-723$

Kawaguchi Y, Kubota Y (1997): GABAergic cell subtypes and their synaptic connections in rat frontal cortex. Cereb Cortex $\underline{7}, 476-486$

Kehrl J, Althaus JC, Showalter HD, Rudzinski DM, Sutton MA, Ueda T (2017): Vesicular Glutamate Transporter Inhibitors: Structurally Modified Brilliant Yellow Analogs. Neurochem Res 42, 1823-1832

Kimura F, Itami C, Ikezoe K, Tamura H, Fujita I, Yanagawa Y, Obata K, Ohshima M (2010): Fast activation of feedforward inhibitory neurons from thalamic input and its relevance to the regulation of spike sequences in the barrel cortex. J Physiol (Lond) $\underline{588,2769-2787}$ 
Kosaka T, Heizmann CW (1989): Selective staining of a population of parvalbumin-containing GABAergic neurons in the rat cerebral cortex by lectins with specific affinity for terminal $\mathrm{N}$-acetylgalactosamine. Brain Res $\underline{483}, 158-163$

Kubota Y, Hattori R, Yui Y (1994): Three distinct subpopulations of GABAergic neurons in rat frontal agranular cortex. Brain Res $\underline{649}, 159-173$

Kwok JCF, Dick G, Wang D, Fawcett JW (2011): Extracellular matrix and perineuronal nets in CNS repair. Dev Neurobiol $\underline{71}, 1073-1089$

Lavallée P, Urbain N, Dufresne C, Bokor H, Acsády L, Deschênes M (2005): Feedforward Inhibitory Control of Sensory Information in Higher-Order Thalamic Nuclei. J Neurosci 25, 7489-7498

Lee S, Hjerling-Leffler J, Zagha E, Fishell G, Rudy B (2010): The largest group of superficial neocortical GABAergic interneurons expresses ionotropic serotonin receptors. J Neurosci $\underline{30}$, 16796-16808

Lodato S, Rouaux C, Quast KB, Jantrachotechatchawan C, Studer M, Hensch TK, Arlotta P (2011): Excitatory projection neuron subtypes control the distribution of local inhibitory interneurons in the cerebral cortex. Neuron $\underline{69}, 763-779$

Ma Y, Hu H, Berrebi AS, Mathers PH, Agmon A (2006): Distinct subtypes of somatostatin-containing neocortical interneurons revealed in transgenic mice. J Neurosci 26, 5069-5082

Markram H, Toledo-Rodriguez M, Wang Y, Gupta A, Silberberg G, Wu C (2004): Interneurons of the neocortical inhibitory system. Nat Rev Neurosci $\underline{5}, 793-807$

Morawski M, Brückner MK, Riederer P, Brückner G, Arendt T (2004): Perineuronal nets potentially protect against oxidative stress. Exp Neurol 188, 309-315

Mulisch M, Welsch U: Romeis - Mikroskopische Technik. 19. Auflage; Springer Spektrum Berlin Heidelberg 2015

Ni B, Rosteck PR, Nadi NS, Paul SM (1994): Cloning and expression of a cDNA encoding a brain-specific $\mathrm{Na}(+)$-dependent inorganic phosphate cotransporter. Proc Natl Acad Sci USA $\underline{\text { 1, }}$ 5607-5611

Nigro MJ, Hashikawa-Yamasaki Y, Rudy B (2018): Diversity and Connectivity of Layer 5 SomatostatinExpressing Interneurons in the Mouse Barrel Cortex. J Neurosci 르, 1622-1633

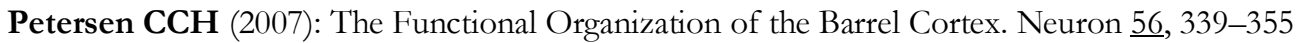

Pfeffer CK, Xue M, He M, Huang ZJ, Scanziani M (2013): Inhibition of inhibition in visual cortex: the logic of connections between molecularly distinct interneurons. Nat Neurosci $\underline{16}, 1068-1076$

Pizzorusso T, Medini P, Berardi N, Chierzi S, Fawcett JW, Maffei L (2002): Reactivation of ocular dominance plasticity in the adult visual cortex. Science 298, 1248-1251

Prieto JJ, Peterson BA, Winer JA (1994): Morphology and spatial distribution of GABAergic neurons in cat primary auditory cortex (AI). J Comp Neurol $\underline{344}, 349-382$

Prönneke A, Scheuer B, Wagener RJ, Möck M, Witte M, Staiger JF (2015): Characterizing VIP Neurons in the Barrel Cortex of VIPcre/tdTomato Mice Reveals Layer-Specific Differences. Cereb Cortex 25, 4854-4868

Ren JQ, Aika Y, Heizmann CW, Kosaka T (1992): Quantitative analysis of neurons and glial cells in the rat somatosensory cortex, with special reference to GABAergic neurons and parvalbumin-containing neurons. Exp Brain Res $\underline{92}, 1-14$

Riddle DR, Purves D (1995): Individual variation and lateral asymmetry of the rat primary somatosensory cortex. J Neurosci 15 , 4184-4195 
Rudy B, Fishell G, Lee S, Hjerling-Leffler J (2011): Three groups of interneurons account for nearly $100 \%$ of neocortical GABAergic neurons. Dev Neurobiol 71, 45-61

Sakakibara A, Hatanaka Y (2015): Neuronal polarization in the developing cerebral cortex. Front Neurosci doi: $10.3389 /$ fnins.2015.00116

Scheuer B: Schichtenspezifische Charakterisierung der VIPcre/tdTomato-Mauslinie mittels neurochemischer Marker. Med. Diss. Göttingen 2015

Schierloh A, Eder M, Zieglgänsberger W, Dodt H-U (2003): Sensory deprivation changes the pattern of synaptic connectivity in rat barrel cortex. Neuroreport $\underline{14}, 1787-1791$

Schubert D, Kötter R, Luhmann HJ, Staiger JF (2006): Morphology, electrophysiology and functional input connectivity of pyramidal neurons characterizes a genuine layer va in the primary somatosensory cortex. Cereb Cortex $\underline{16}, 223-236$

Schubert D, Kötter R, Staiger JF (2007): Mapping functional connectivity in barrel-related columns reveals layer- and cell type-specific microcircuits. Brain Struct Funct 212, 107-119

Simons DJ (1978): Response properties of vibrissa units in rat SI somatosensory neocortex. J Neurophysiol $\underline{41}$, 798-820

Sohal VS, Zhang F, Yizhar O, Deisseroth K (2009): Parvalbumin neurons and gamma rhythms enhance cortical circuit performance. Nature $\underline{459}, 698-702$

Staiger JF, Zilles K, Freund TF (1996): Innervation of VIP-immunoreactive neurons by the ventroposteromedial thalamic nucleus in the barrel cortex of the rat. J Comp Neurol 367, 194-204

Staiger JF, Freund TF, Zilles K (1997): Interneurons immunoreactive for vasoactive intestinal polypeptide (VIP) are extensively innervated by parvalbumin-containing boutons in rat primary somatosensory cortex. Eur J Neurosci $\underline{9}, 2259-2268$

Staiger JF, Masanneck C, Schleicher A, Zuschratter W (2004): Calbindin-containing interneurons are a target for VIP-immunoreactive synapses in rat primary somatosensory cortex. J Comp Neurol 468, 179-189

Staiger JF, Zuschratter W, Luhmann HJ, Schubert D (2009): Local circuits targeting parvalbumin-containing interneurons in layer IV of rat barrel cortex. Brain Struct Funct 214, 1-13

Staiger JF, Möck M, Proenneke A, Witte M (2015): What types of neocortical GABAergic neurons do really exist? e-Neuroforum $\underline{6}, 49-56$

Steullet P, Cabungcal J-H, Bukhari SA, Ardelt MI, Pantazopoulos H, Hamati F, Salt TE, Cuenod M, Do KQ, Berretta S (2018): The thalamic reticular nucleus in schizophrenia and bipolar disorder: role of parvalbumin-expressing neuron networks and oxidative stress. Mol Psychiatry 23, 2057-2065

Szabadics J, Varga C, Molnár G, Oláh S, Barzó P, Tamás G (2006): Excitatory effect of GABAergic axoaxonic cells in cortical microcircuits. Science $311,233-235$

Takamori S, Riedel D, Jahn R (2000): Immunoisolation of GABA-Specific Synaptic Vesicles Defines a Functionally Distinct Subset of Synaptic Vesicles. J Neurosci 20, 4904-4911

Tan X, Shi S-H (2013): Neocortical neurogenesis and neuronal migration. Wiley Interdiscip Rev Dev Biol 2 , 443-459

Tewari BP, Chaunsali L, Campbell SL, Patel DC, Goode AE, Sontheimer H (2018): Perineuronal nets decrease membrane capacitance of peritumoral fast spiking interneurons in a model of epilepsy. Nature Commun $\underline{9}, 4724$

Thompson CM, Davis E, Carrigan CN, Cox HD, Bridges RJ, Gerdes JM (2005): Inhibitor of the glutamate vesicular transporter (VGLUT). Curr Med Chem 12, 2041-2056 
Tremblay R, Lee S, Rudy B (2016): GABAergic Interneurons in the Neocortex: From Cellular Properties to Circuits. Neuron 11, 260-292

van Brederode JF, Helliesen MK, Hendrickson AE (1991): Distribution of the calcium-binding proteins parvalbumin and calbindin-D28k in the sensorimotor cortex of the rat. Neuroscience $44,157-171$

Walker F, Möck M, Feyerabend M, Guy J, Wagener RJ, Schubert D, Staiger JF, Witte M (2016): Parvalbumin- and vasoactive intestinal polypeptide-expressing neocortical interneurons impose differential inhibition on Martinotti cells. Nature Commun 7,13664

Wang D, Fawcett J (2012): The perineuronal net and the control of CNS plasticity. Cell Tissue Res $\underline{349}$, 147160

Weigel A, Schild D, Zeug A (2009): Resolution in the ApoTome and the confocal laser scanning microscope: comparison. J Biomed Opt $\underline{14}, 014022$

Wonders CP, Anderson SA (2006): The origin and specification of cortical interneurons. Nat Rev Neurosci $\underline{7}$, 687-696

Woodruff A, Xu Q, Anderson SA, Yuste R (2009): Depolarizing effect of neocortical chandelier neurons. Front Neural Circuits $\underline{3}, 15$

Woolsey TA, Van der Loos H (1970): The structural organization of layer IV in the somatosensory region (SI) of mouse cerebral cortex. The description of a cortical field composed of discrete cytoarchitectonic units. Brain Res $\underline{17}, 205-242$

Xu Q, Cobos I, Cruz EDL, Rubenstein JL, Anderson SA (2004): Origins of Cortical Interneuron Subtypes. J Neurosci 24, 2612-2622

Xu X, Roby KD, Callaway EM (2010): Immunochemical characterization of inhibitory mouse cortical neurons: three chemically distinct classes of inhibitory cells. J Comp Neurol $\underline{518}$, 389-404

Yamada J, Jinno S (2013): Spatio-temporal differences in perineuronal net expression in the mouse hippocampus, with reference to parvalbumin. Neuroscience 253, 368-379

Yavorska I, Wehr M (2016): Somatostatin-Expressing Inhibitory Interneurons in Cortical Circuits. Front Neural Circuits doi: 10.3389/fncir.2016.00076

Young NM, Williams RE (1985): Assignment of lectins specific for D-galactose or N-acetyl-D-galactosamine to two groups, based on their circular dichroism. Can J Biochem Cell Biol 63, 268-271

Zander J-F, Münster-Wandowski A, Brunk I, Pahner I, Gómez-Lira G, Heinemann U, Gutiérrez R, Laube G, Ahnert-Hilger G (2010): Synaptic and vesicular coexistence of VGLUT and VGAT in selected excitatory and inhibitory synapses. J Neurosci $\underline{30}, 7634-7645$ 
